\begin{abstract}
Title of Document:

AN INVESTIGATION INTO THE ADVANTAGES OF IN-SPACE PROPELLANT RE-SUPPLY

Michael David Scher, Master of Science, 2006

Directed By:

Professor Robert H. Tolson

Department of Aerospace Engineering
\end{abstract}

In-space propellant re-supply has been proposed to extend space vehicle lifetimes, but only qualitative arguments supporting development have been made; this study focuses on quantitative support. Two basic concepts for propellant resupply are propellant transfer and tank replacement. A quantitative assessment and comparison to no re-supply alternatives is necessary to choose the most cost-effective architecture. The analysis is primarily accomplished through the use of a new tool that facilitates propulsion system sizing and broad trade studies. The program was co-created by the author under a grant from NASA Langley Research Center and has been used at NASA Langley for lunar architecture studies to support the Vision for Space Exploration. For less than 100 missions, the preferred scenario is without resupply, using direct Earth reentry. For more than 100 missions, tank replacement requires $38 \%$ fewer missions than propellant transfer to become more cost effective than no re-supply. 


\title{
AN INVESTIGATION INTO THE ADVANTAGES OF IN-SPACE PROPELLENT RE-SUPPLY
}

\author{
By \\ Michael David Scher \\ Thesis submitted to the Faculty of the Graduate School of the \\ University of Maryland, College Park, in partial fulfillment \\ of the requirements for the degree of \\ Master of Science \\ 2006
}

Advisory Committee:

Professor Robert H. Tolson, Co-chair

Professor William Fourney, Co-chair

Professor David Akin

Professor Christopher Cadou 
(C) Copyright by

Michael David Scher

2006 


\section{Dedication}

I would like to dedicate my work to my family and friends who have supported me in all of my endeavors, academic or otherwise. You have all served as inspiration for me to strive towards high goals and achievements. I especially would like to recognize my wife, Jennifer, as my reason for succeeding. 


\section{Acknowledgements}

I would like to thank my advisor, Dr. Tolson, for sharing his knowledge and experience with me during this process. I extend a special thanks to my colleagues and friends at NASA Langley Research Center who have served as mentors in my professional development, in particular Pat Troutman and Dave North. Also, I am grateful to the University of Maryland, National Institute of Aerospace, and NASA Langley for providing me with this unique opportunity. 


\section{Table of Contents}

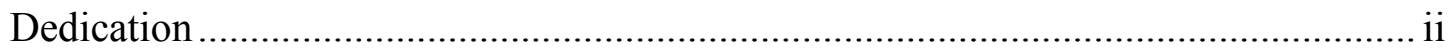

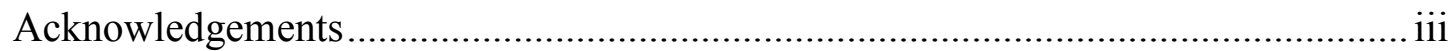

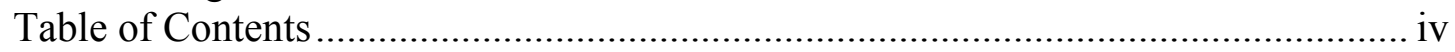

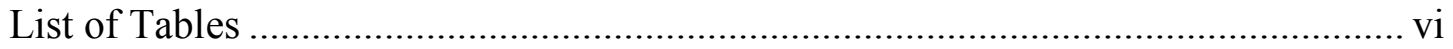

List of Figures ......................................................................................... vii

Chapter 1: Introduction ..................................................................................... 1

Chapter 2: Mission Concepts of Operation............................................................... 5

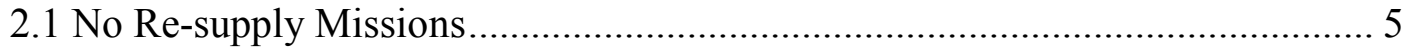

2.1.1 No Re-supply Mission Elements ............................................................ 5

2.1.2 No Re-supply Scenario Descriptions .......................................................... 6

2.2 Propellant Re-supply Elements.................................................................. 7

2.2.1 Propellant Transfer Mission Elements...................................................... 8

2.2.2 Tank Replacement Mission Elements......................................................... 9

2.2.3 Propellant Re-Supply Scenario Descriptions.............................................. 9

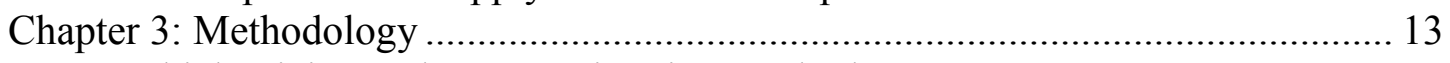

3.1 Vehicle Sizing and Mass Estimating Method ..................................................... 13

3.2 Vehicle Sizing and Mass Estimating Assumptions .......................................... 17

3.3 Velocity Change Maneuvers .................................................................... 21

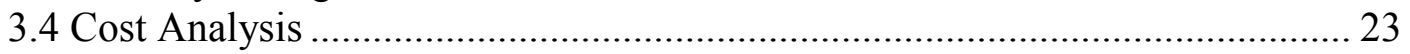

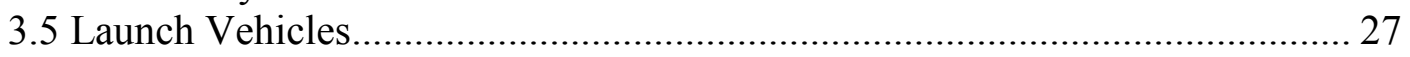

3.6 Analysis Procedure Automation ............................................................... 28

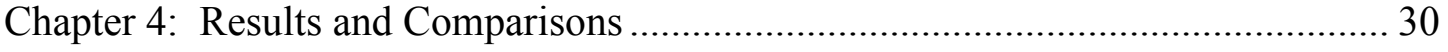

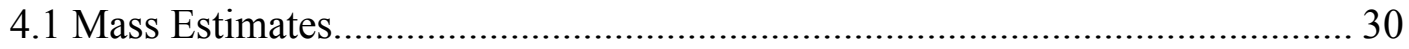

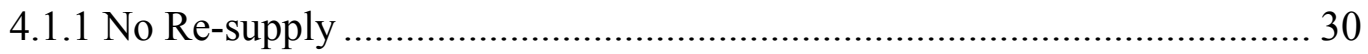

4.1.2 Propellant Transfer............................................................................. 31

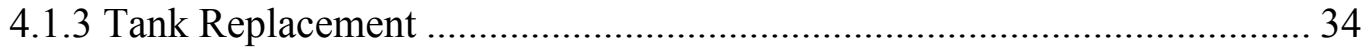

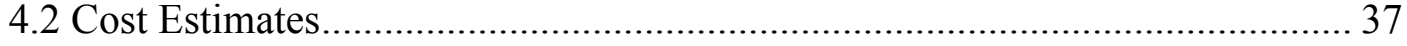

4.2.1 Development Costs ................................................................................. 37

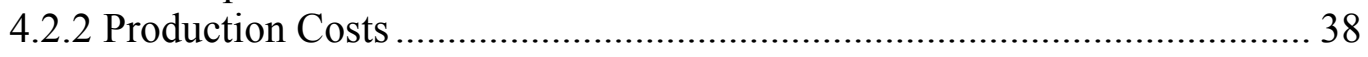

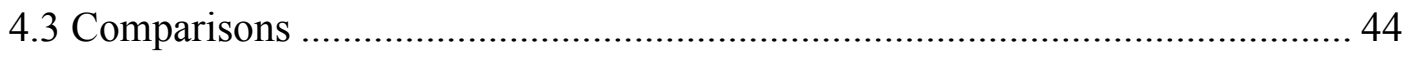

4.3.1 No Re-supply Architectures...................................................................... 44

4.3.2 Propellant Transfer versus Tank Replacement .......................................... 46

4.3.3 No Re-supply Versus Re-supply......................................................... 48

4.3.4 Comparison Summary …………………………................................. 50

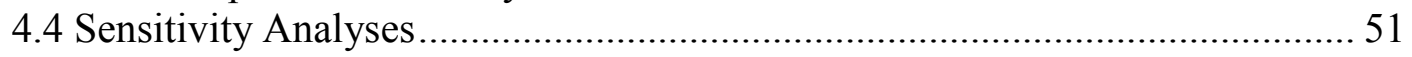

4.4.1 Propellant Transfer Loss Variation ......................................................... 52

4.4.2 Variation of Percentage of Additional Hardware for Tank Replacement.. 54

4.4.3 Inert Mass Variation ................................................................................ 56

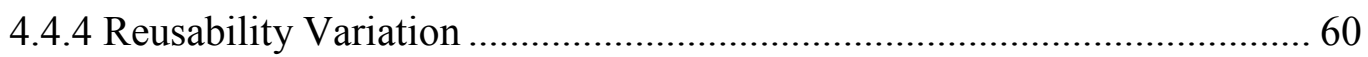

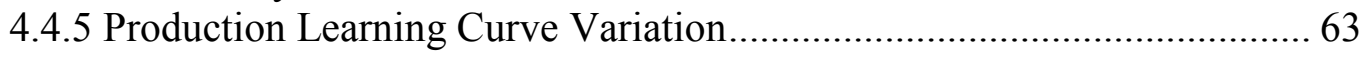

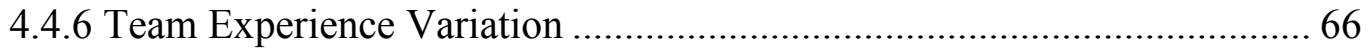

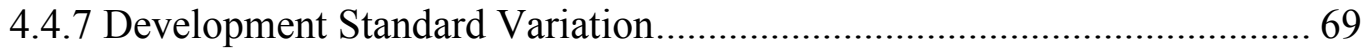




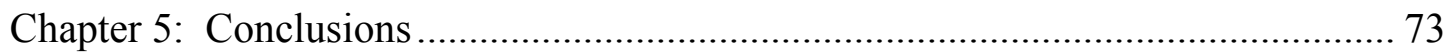

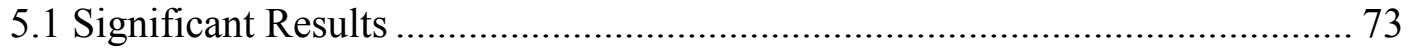

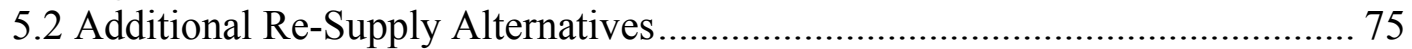

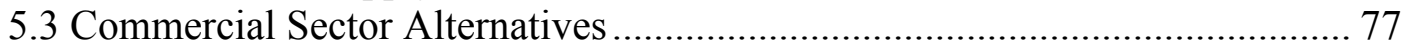

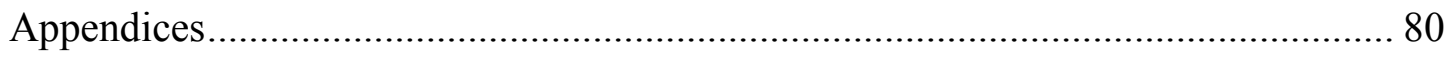

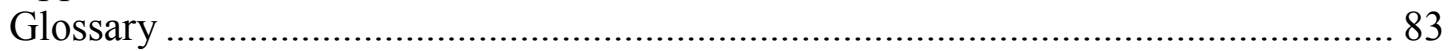

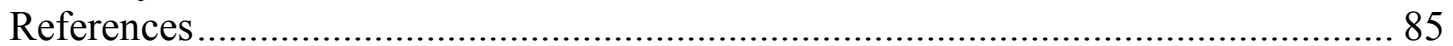




\section{List of Tables}

Table 1: Comparison for Atlas-Centaur III and Delta IV upper stages. .................... 16

Table 2: Vehicle sizing and mass estimating assumptions................................... 19

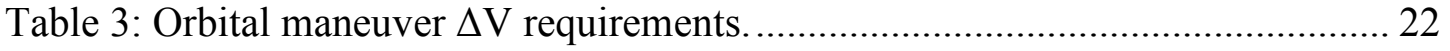

Table 4: Lunar-bound and Earth-bound velocity change totals................................ 23

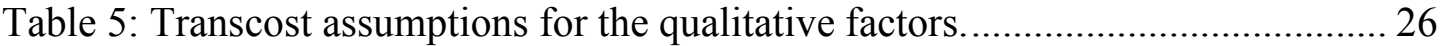

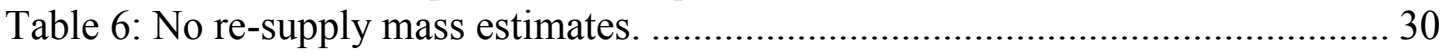

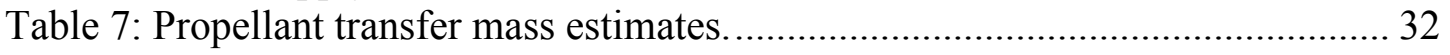

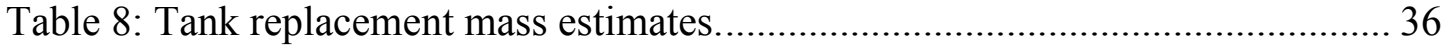

Table 9: Development costs for each element in all architectures............................. 38 


\section{List of Figures}

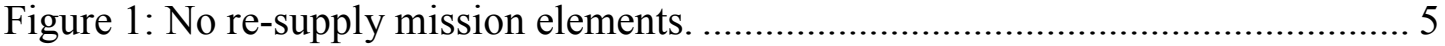

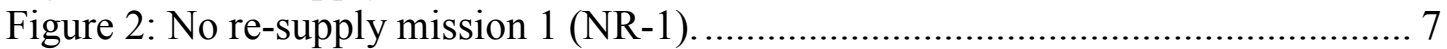

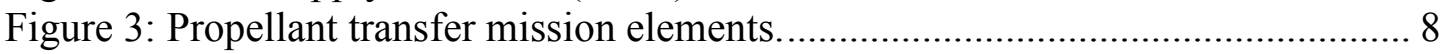

Figure 4: Tank replacement mission elements....................................................... 9

Figure 5: Part 1 of propellant re-supply mission scenario. ........................................ 10

Figure 6: Part 2 of propellant re-supply mission scenario. ....................................... 11

Figure 7: Sizing methods used for each subsystem module.................................... 14

Figure 8: Subsystem breakdown of actual and modeled S-IVb.............................. 16

Figure 9: CAD model of PMre and two TRMs. ................................................... 20

Figure 10: Production numbers for propellant transfer......................................... 40

Figure 11: Production numbers for tank replacements............................................. 40

Figure 12: No re-supply total and average production versus number of missions. .. 43

Figure 13: Percent increase in production cost over PT-0 and TR-0 ...................... 43

Figure 14: Average total cost vs. number of missions for no re-supply scenarios.... 45

Figure 15: Average total cost vs. number of missions for re-supply scenarios......... 47

Figure 16a: Comparison of average total cost for no re-supply and re-supply........... 49

Figure 16b: Comparison of average total cost for no re-supply and re-supply. ......... 49

Figure 17: Location of crossover points for different amounts of transfer loss.......... 53

Figure 18: Location of crossover points for different amounts of added hardware.... 55

Figure 19: No re-supply scenario sensitivity to inert mass.................................... 57

Figure 20: Propellant transfer scenario sensitivity to inert mass. .............................5 59

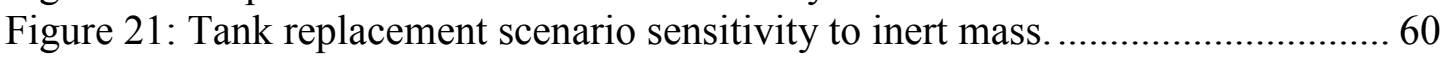

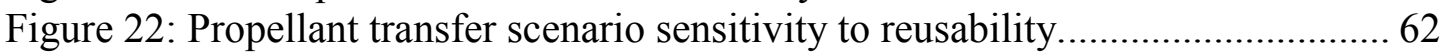

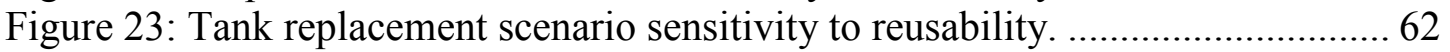

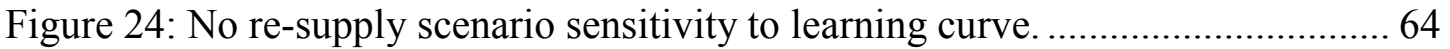

Figure 25: Propellant transfer scenario sensitivity to learning curve. ...........................65

Figure 26: Tank replacement scenario sensitivity to learning curve. .........................6 66

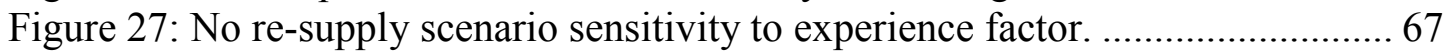

Figure 28: Propellant transfer scenario sensitivity to experience factor......................6 68

Figure 29: Tank replacement scenario sensitivity to experience factor.....................6 69

Figure 30: No re-supply scenario sensitivity to development factor. ........................ 70

Figure 31: Propellant transfer scenario sensitivity to development factor. ................ 71

Figure 32: Tank replacement scenario sensitivity to development factor. ................. 72 


\section{Chapter 1: Introduction}

Since the beginning of aviation, engineers have been exploring methods to extend the range of aircraft. The efforts culminated in a successful aerial refueling in 1923, increasing the range of an individual aircraft to a nearly limitless capability; this complex procedure has become a routine component of military flights ${ }^{1}$. Similarly, in-space propellant resupply has been proposed as a means of extending the lifetime of space vehicles ${ }^{2}$.

Beginning with the framers of the Apollo manned lunar landing program, the advantages of in-space re-supply have been considered. The Earth orbit rendezvous (EOR) architecture mode that was contemplated for the first lunar landing incorporated a tanker vehicle to transfer liquid oxygen (LOX) to the S-IVb before trans-Lunar injection (TLI). This architecture alternative would have decreased the net lift-off weight of the manned launch, but the EOR architecture was not adopted for Apollo ${ }^{3}$. The Russians revisited the concept of propellant re-supply with their Progress Module, which has been demonstrating the ability to deliver consumables to space stations in low Earth orbit (LEO) since $1978^{2}$. Additionally, the NASA Space Shuttle has been involved in several on-orbit experiments. The experiments have supported evaluation and understanding of the complicated fluid dynamics and thermodynamics involved in transferring Earth-storable or cryogenic propellants. However, analyses have focused on the necessary subsystem apparatus to perform inspace propellant re-supply. To date, the work has been driven by qualitative arguments to support the technological development of propellant re-supply. 
Within the body of knowledge about re-supplying space vehicles, two basic concepts have been offered as the means to achieve in-space propellant re-supply. The two methods are propellant transfer and tank replacement ${ }^{4}$. Propellant transfer is the direct exchange of propellant from a tanker supply tank to a receiving tank; this method generally includes the use of positive displacement devices, draining via artificial gravity, or gas pressurant. Additionally, direct propellant transfer of cryogenic propellants requires the slow chill-down of the receiving tank to avoid thermal stress fractures. The chill-down is accomplished through a sacrificial amount of propellant that is allowed to boil off while reducing the temperature of the receiving tank; the amount of propellant expected to boil off in this manner is referred to as the propellant transfer loss.

The tank replacement method is an indirect re-supply method because it only requires the removal of an empty propellant tank, which is replaced with a new, full tank. The replacement process could either be telerobotic or astronaut-assisted. With both approaches, the method will likely require some additional hardware to prevent the tank and feed line connections from failing in the vacuum of space. Since most propellant tanks are highly integrated into the main structure of the vehicle to transfer axial loads, additional structure will be needed to accommodate the removal of the tanks. The added hardware and structure is an increase in the vehicle dry mass; it is referred to as additional tank replacement hardware. For an in-space propulsion stage, it is necessary to re-supply the pressurant tanks as well as the fuel and oxidizer tanks. As an alternative to replacing the tanks individually, a tank replacement module (TRM) could be used. The TRM would consist of fuel and oxidizer tanks, 
pressurant tanks, propellant feed system, support structure, and other limited subsystems. The TRM would be able to directly dock with the vehicle it is resupplying.

Though both propellant transfer and tank replacement methods have significant technical difficulties, it is necessary to conduct an "apples to apples" quantitative comparison and assessment. If only one method can be pursued due to financial and manpower limitations, it is ideal to develop the method that provides the greatest enhancement of mission performance. Additionally, the inherent losses of each re-supply method could be high. In which case, it is necessary to understand the loss mitigation as a result of additional development effort.

The analysis is primarily accomplished through the use of a new, advanced tool. The Space Propulsion Sizing Program (SPSP) is used to facilitate in-space propulsion system sizing and broad system-level trade studies. The program was cocreated by the author under a grant from the Space Mission Analysis Branch at NASA Langley Research Center. The original design objectives for SPSP were to satisfy the need for a reliable and easy-to-use tool for rapid, high-level trade study and analysis. The capabilities of SPSP were ideally suited to the design and analysis necessary to understand the advantages of in-space propellant re-supply.

The SPSP software has been used at NASA Langley for lunar architecture and transportation studies to support the Vision for Space Exploration that was outlined by President Bush in January 2004. Since the rollout of the first version in October 2004, several studies have been conducted using SPSP. The largest studies were the Phased Capability Advanced Technologies (PCAT) architecture study in early 2005 
and the three month Exploration Systems Architecture Study (ESAS) that came later in 2005. In PCAT, the SPSP was the primary tool used to model in-space propulsion stages and lunar landers. The lander version of SPSP was used to heavily support the ESAS lander design and analysis. In addition to these larger projects, the SPSP has been relied upon as the only tool for a propellant transfer and aggregation study and a non-toxic propellant study. Recently, the Lunar Architecture Requirements Preparatory Study has used SPSP for comparison and validation of other software. The lunar architecture re-supply study conducted here is independent of NASA efforts. 


\section{Chapter 2: Mission Concepts of Operation}

\subsection{No Re-supply Missions}

This section discusses the no re-supply options. The no re-supply options do not use any reusable vehicles and are similar to Apollo and currently proposed lunar architectures.

\subsubsection{No Re-supply Mission Elements}

The primary elements for the no re-supply lunar mission scenarios are illustrated in figure 1. The elements are a crew exploration vehicle (CEV), a manned launch vehicle, an expendable in-space propulsion module (PM-E), an unmanned launch vehicle, and an expendable single stage lander. The details of both the manned and unmanned launch vehicles are discussed in section 3.3. The single stage lander was selected for simplicity and comparability to the re-supply scenarios, as explained in section 2.2.1.

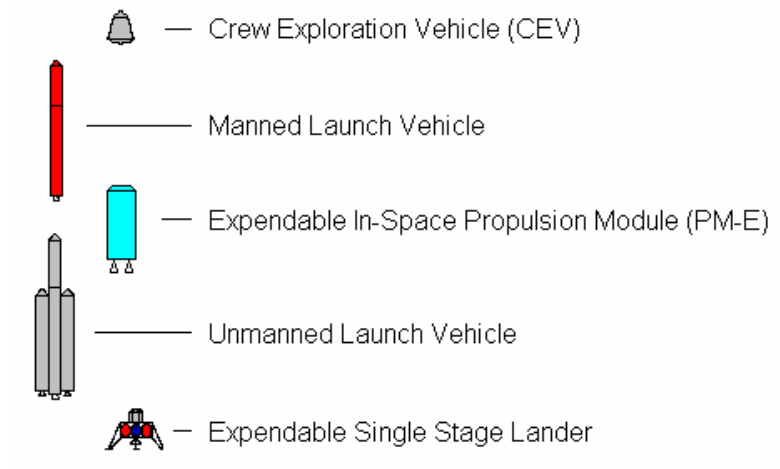

Figure 1: No re-supply mission elements. 


\subsubsection{No Re-supply Scenario Descriptions}

Three no re-supply mission scenarios were designed with similarity to the NASA Vision for Space Exploration Lunar Architecture ${ }^{5}$ and to the re-supply lunar architectures considered here, which incorporate the use of Earth orbit rendezvous and lunar orbit rendezvous each mission. The specific velocity change maneuvers $(\Delta V s)$ and the parking orbits around Earth and the Moon are described in section 3.1.

No re-supply mission 1 (NR-1) is illustrated in figure 2. The number of launches illustrated is to represent the most number of launches that may be required; the actual number of launches is dependent on the specific vehicle designs. After all of the cargo launches, the CEV is delivered into LEO by the manned launch vehicle, where rendezvous and docking is performed with the elements already in orbit. The TLI and Lunar orbit insertion (LOI) maneuvers are performed using the PM-E. The entire crew egresses to the lander and performs a powered descent to and lands on the lunar surface. Upon completion of the lunar surface portion of the mission, the crew launches in the lander into low Lunar orbit (LLO) to rendezvous and dock with the assets that are loitering in orbit. After jettison and disposal of the lander, the transEarth injection (TEI) and propulsive Earth orbit capture (EOC) maneuvers are performed by the PM-E. The PM-E is disposed, and the crew performs the entry, descent, and landing (EDL) in the CEV. 


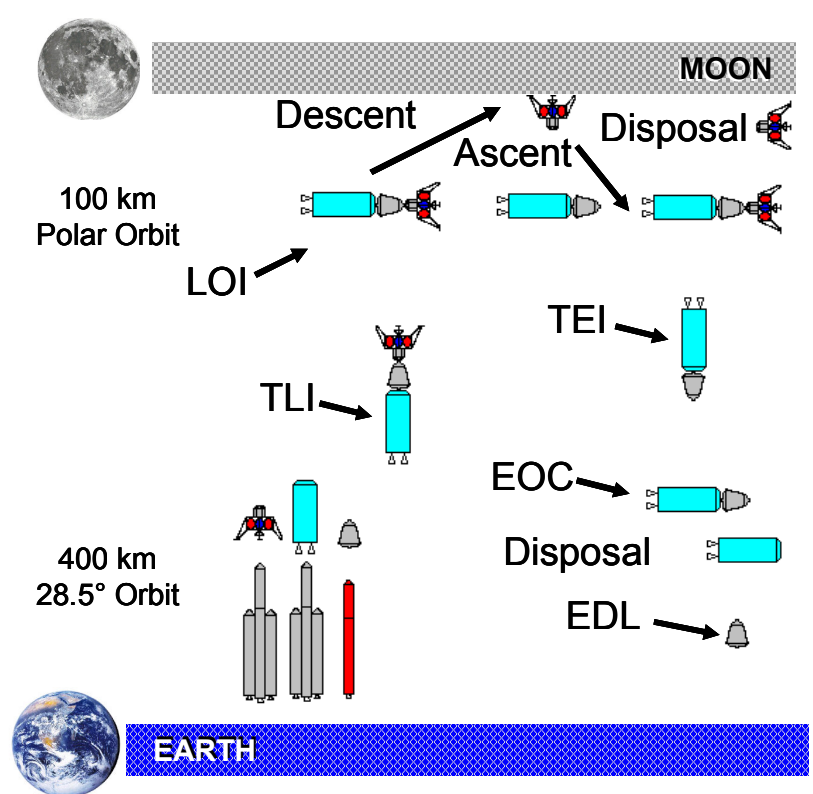

Figure 2: No re-supply mission 1 (NR-1).

The no re-supply 2 (NR-2) and no re-supply 3 (NR-3) missions are similar to NR-1 as far as the elements and concept of operations. However, for NR-2 there are two identical PM-Es to divide the maneuvers. The TLI and LOI maneuvers are performed by the first PM-E; whereas, the TEI and EOC maneuvers are performed by the second PM-E. The PM-E 1 and PM-E 2 are expended in LLO and LEO, respectively. The difference between NR-1 and NR-3 is that the EOC maneuver has been removed. Instead, the CEV performs a direct EDL after the TEI maneuver.

\subsection{Propellant Re-supply Elements}

This section covers the two re-supply methods. The two methods use some reusable and some expendable vehicles within an architecture similar to the no resupply options. 


\subsubsection{Propellant Transfer Mission Elements}

The elements for the propellant transfer scenarios are similar to those of the no re-supply scenarios, shown in figure 3. For the propellant transfer missions, additional vehicle elements have been added or changed as follows: a reusable inspace propulsion module (PM-R), a reusable lander, an expendable in-space propulsion module (PM-E), and a propellant transfer module (PTM). The PM-R is only used to transport the CEV from LEO to LLO and back. The PM-E is used to transport the lander and PTMs from LEO to LLO, where the PM-E and PTMs are discarded.

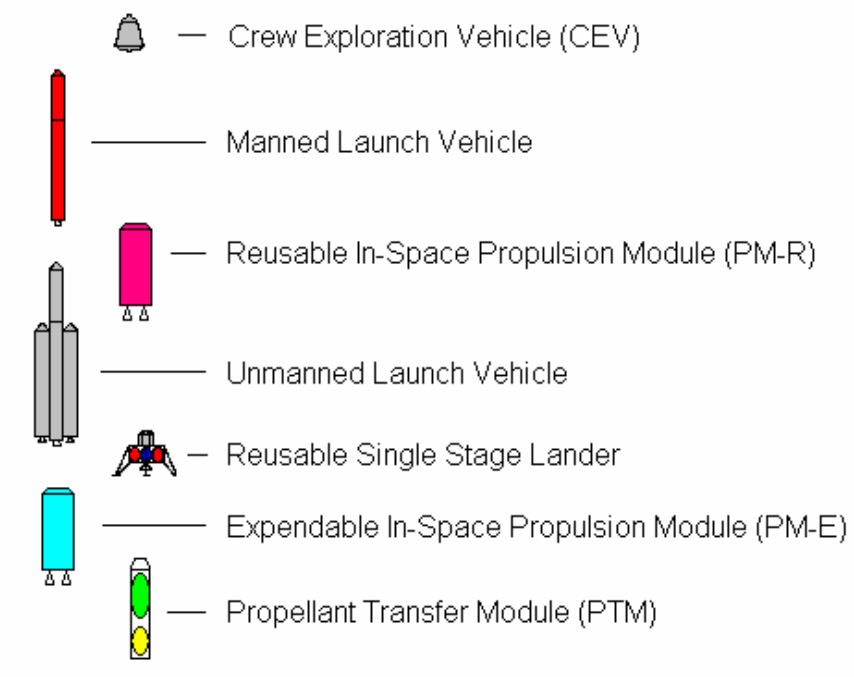

Figure 3: Propellant transfer mission elements.

A single stage lander is selected for re-supply to facilitate its reusability. For example, a two stage lander could not be completely reusable because the descent stage would be left empty on the surface. Unless both the ascent and descent stages were refilled, a new descent stage would have to be sent out to LLO. If both stages were to be re-supplied, extreme inefficiencies would be introduced due to the difficulty of delivering PTMs to the lunar surface and the added expense of the 
descent stage using this propellant to lift off from the surface and re-dock with the ascent stage. Despite the gains of dividing the $\Delta \mathrm{Vs}$ during crewed lunar missions, the added propellant requirements would more than erode any such advantage.

\subsubsection{Tank Replacement Mission Elements}

The types of mission elements for the tank replacement missions, shown in figure 4, are analogous to the elements used for propellant transfer. Instead of a PTM, the tank replacement scenarios use the TRM, which is used to replace expended propellant tanks on the reusable lander and PM-R. As in the propellant transfer scenarios, the PM-E delivers the TRMs and reusable lander to LLO, and the PM-R is only for transporting the CEV to and from LLO.

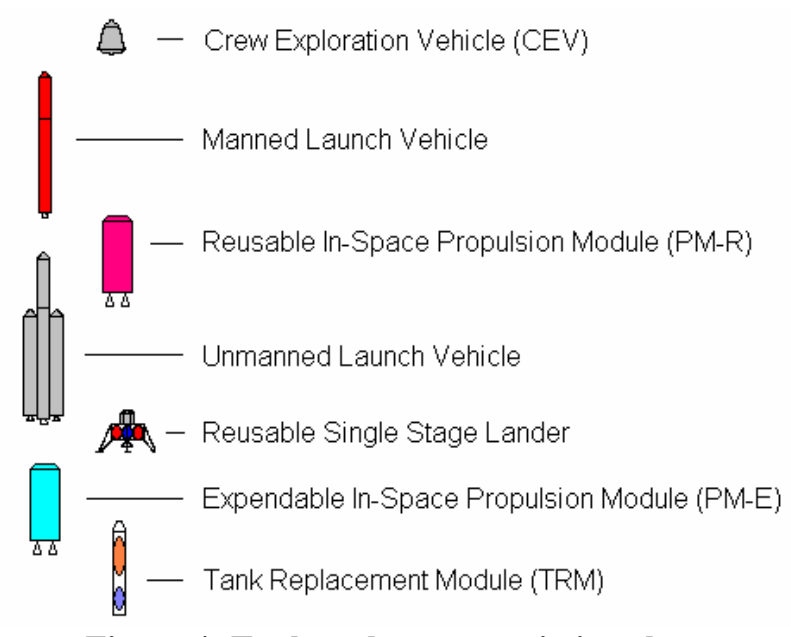

Figure 4: Tank replacement mission elements.

\subsubsection{Propellant Re-Supply Scenario Descriptions}

The propellant re-supply scenarios are comprised of two parts. The first part is when the reusable elements are launched and used for the first time in a campaign; the second part consists of one or more follow-on missions within the same 
campaign, in which only expendable elements are launched and the reusable elements are re-supplied. A campaign is defined as a series of missions which all use the same reusable elements. The scenarios are the same for both methods of propellant resupply; the distinction comes from the particular elements that are being used. In figures 5 and 6 , respectively, the two parts of a campaign are illustrated.

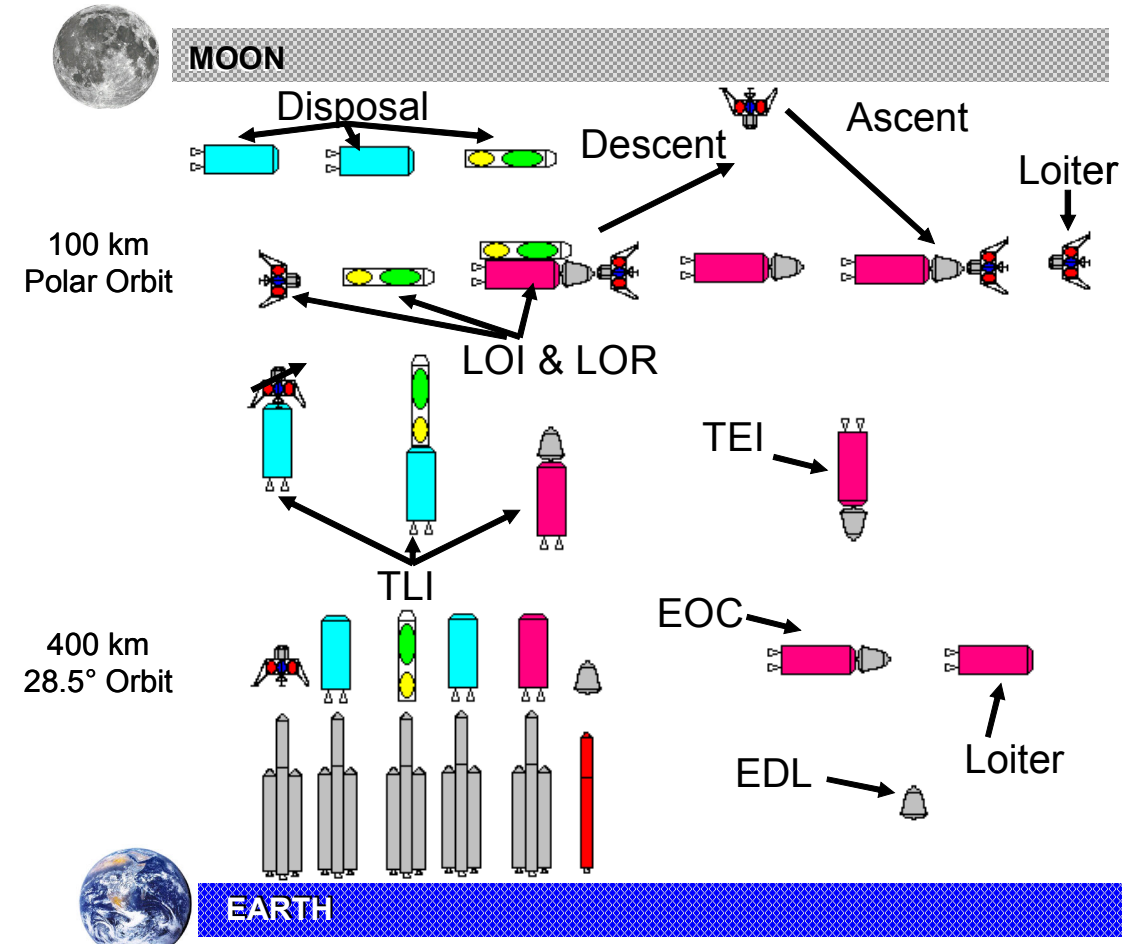

Figure 5: Part 1 of propellant re-supply mission scenario.

For the first mission in a campaign, the lander and PM-R are launched fully fueled. Then, the appropriate number of re-supply modules, either PTMs or TRMs, are launched to re-supply the PM-R for return to Earth from LLO, and the necessary number of PM-Es is launched to deliver the lander and re-supply modules to LLO. The last launch is the crew in the CEV. Then, in several groups, the lander and resupply modules are delivered to LLO, where the CEV and PM-R rendezvous with 
them. In the lander, the crew descends to the lunar surface to complete the surface operations, and then they ascend to rendezvous with the loitering assets in LLO. Either while the crew is on the surface or shortly before descent, the PM-R is resupplied by the appropriate re-supply modules; the expended PM-Es and re-supply modules are then discarded. The lander remains in LLO until the next mission in the current campaign, while the CEV and PM-R return to LEO. After the CEV completes the EDL, the PM-R loiters in LEO until the next mission.

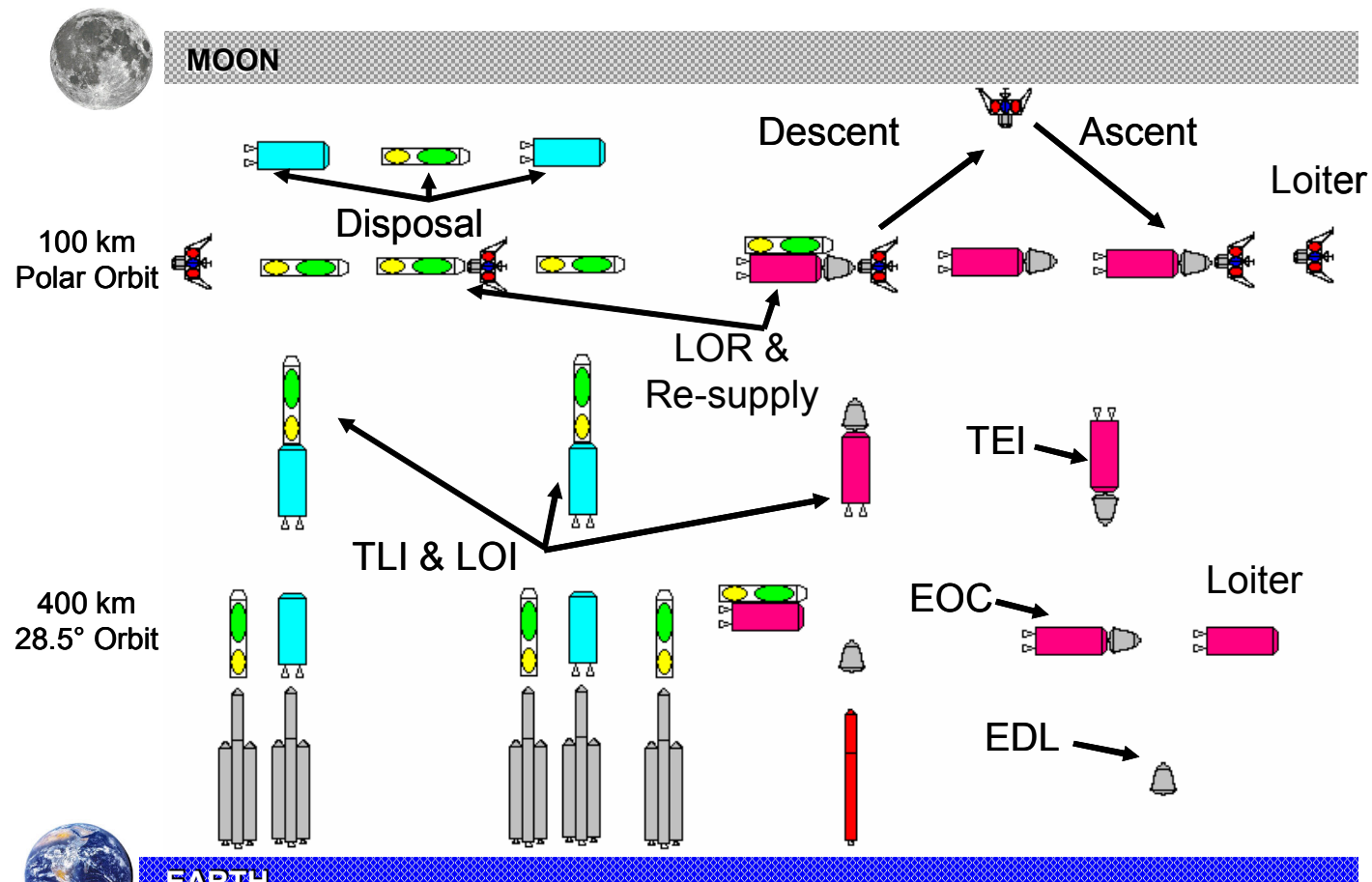

Figure 6: Part 2 of propellant re-supply mission scenario.

For the subsequent missions in the campaign, the number of re-supply modules that are needed to complete the mission is increased. The PM-R is resupplied in LEO prior to the beginning of the mission and again in LLO during the mission; the lander is also re-supplied in LLO. As before, the re-supply modules are delivered to LLO by the PM-Es. The CEV is then brought to LLO by the newly re- 
supplied PM-R. The lander is re-supplied, and the remainder of the mission completes as before. The campaign continues until the lander and PM-R can no longer be reused and must be disposed of. At this point, a new campaign begins with part 1.

It is possible to reduce the number of re-supply modules for later missions within a campaign by delivering extra modules during earlier missions. For example, if a single PM-E is designed to deliver multiple re-supply modules. Then, if the demand for re-supply modules is lower than the capability of the system, one or more extra modules could be delivered. This can be useful for reducing the future demand for additional launches and elements during a campaign, as well as creating redundancy and reserves in the event of problems. 


\section{Chapter 3: Methodology}

\subsection{Vehicle Sizing and Mass Estimating Method}

The vehicle sizing and mass estimating was accomplished using the Space Propulsion Sizing Program (SPSP). The SPSP was developed using a combination of the best mass estimating methods available. The combined methods and internal functionality allows the user to quickly progress from an initial design concept to a detailed mass breakdown and geometry sizing. The simple interface and reliable methodology provides a rapid trade-study environment for individual element and large-scale architecture analysis ${ }^{6}$.

The methods of mass estimating that were selected include bottom-up calculations, mass estimating relationships, and historical database regressions. Each subsystem module of the program was developed using one or more of each type of method. Additionally, the user can override any approach or isolate one preferred method within the subsystem estimates. Figure 7 shows the combination of sizing methods for each of the subsystems. The interface was developed in a Microsoft Excel workbook, utilizing Visual Basic routines. 


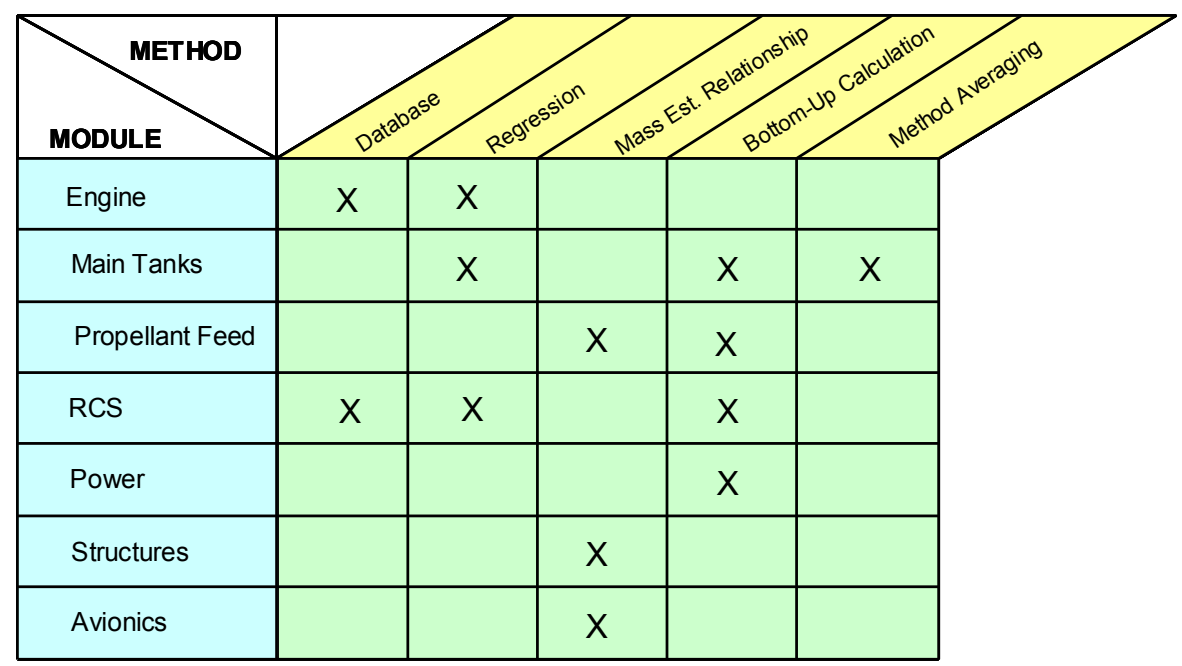

Figure 7: Sizing methods used for each subsystem module.

In addition to mass estimating, SPSP generates a vehicle size estimate. The basic vehicle geometry is based on the most significant subsystems: structures, main tanks, and engine. However, for more advanced sizing, other subsystem dimensions are included. The simplest geometry output is an automatically generated 2dimensional cross section. For more advanced, 3-dimensional outputs, the data generated by SPSP can be supplied to several different computer-aided design (CAD) software packages. Pro-Engineer is completely integrated into SPSP, but the capability of geometry sizing has also been demonstrated in I-DEAS and MATLAB.

One of the design requirements for SPSP was long-term adaptability. This was necessary to allow SPSP to be altered and expanded. As a result, additional internal capabilities and spin-off tools were developed to supplement the main SPSP tool; the supplements were necessary because several studies required new and additional capabilities that were based on or used in conjunction with existing SPSP capabilities. The spin-off tools that were developed are as follows: staging tool with optimizer, tanker vehicle sizing tool, lander sizing tool, and launch vehicle design and 
analysis tool. Each of these tools was built from the main SPSP. The staging tool creates additional copies of SPSP, which can be individually modified; the multistage vehicle can then be optimized. The tanker sizing tool was created by removing the main engine module and has been used to support propellant transfer and propellant aggregation studies. The lander sizing tool is the main SPSP with the addition of structure equal to $3 \%$ of the touchdown mass to account for landing gear mass. The landing gear addition is based on the Apollo lander, which had a landing gear mass equal to $2.9 \%$ of the mass that landed on the lunar surface ${ }^{7}$. Launch vehicle design and analysis tool is the staging tool interfaced with a MATLAB-based integration and targeting scheme. The main SPSP was also modified to include boil off propellant mass estimates and complicated maneuvers with different payload masses through different $\Delta \mathrm{Vs}$.

Most importantly, the final version of the main SPSP tool was benchmarked against historical vehicles to demonstrate the reliability of mass estimates and geometry sizing. The S-IVb, Atlas-Centaur III, and Delta IV upper stages were used for validation. Figure 8 shows the comparison of the actual and SPSP estimated S$\mathrm{IVb}$ inert masses. The primary difference is the conservative estimate by SPSP of the residual propellant loads. Similarly, table 1 compares the actual and SPSP models for the Atlas-Centaur III and Delta IV upper stages. The small errors are caused by the use of mass estimating relationships that were developed based on older technologies. The errors are sufficiently small, which demonstrate that SPSP models are realistic. 


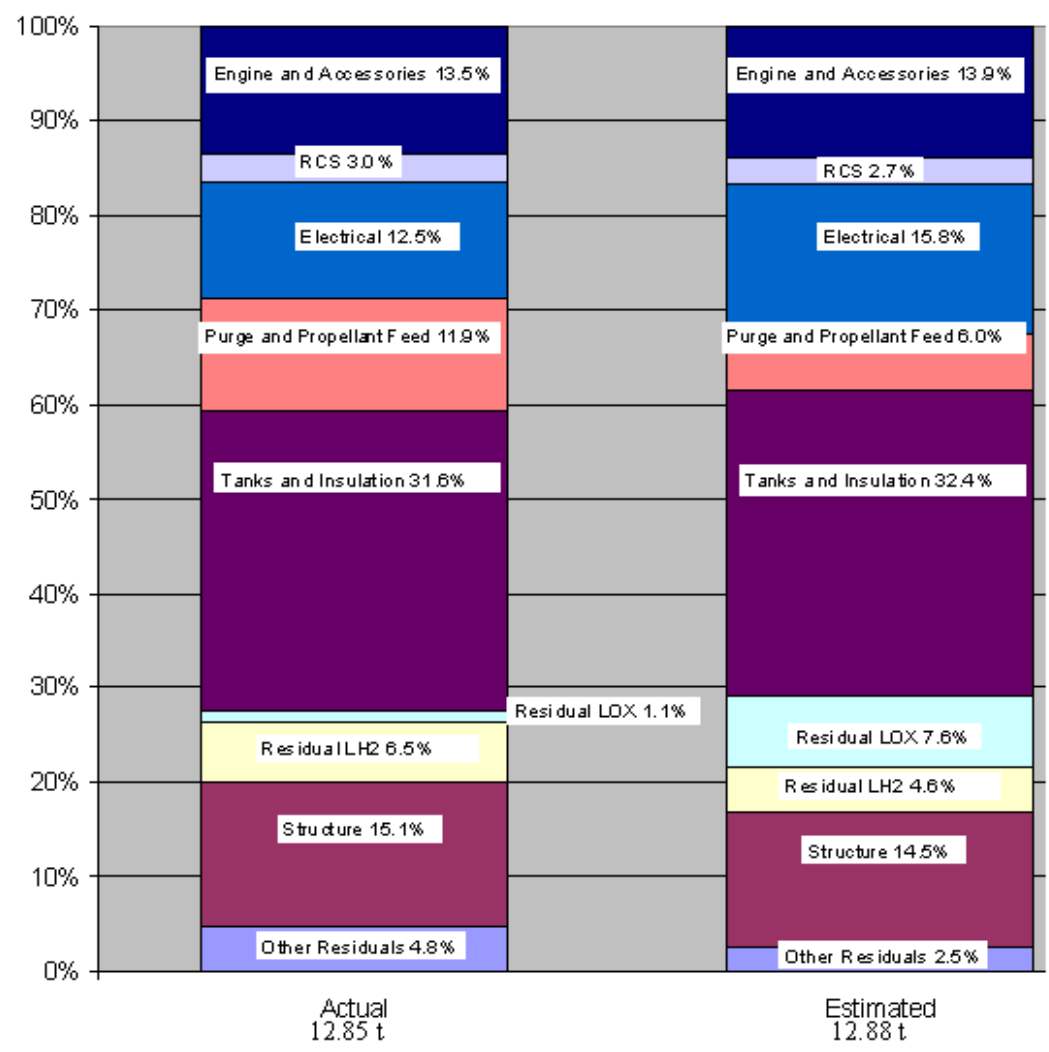

Figure 8: Subsystem breakdown of actual and modeled S-IVb.

Table 1: Comparison for Atlas-Centaur III and Delta IV upper stages.

\begin{tabular}{|c|c|c|c|}
\hline & Actual Value & SPSP Estimate & \% Error \\
\hline \multicolumn{4}{|c|}{ Atlas-Centaur III } \\
\hline Gross Mass (kg) & 18960 & 19322 & $1.91 \%$ \\
\hline Inert Mass (kg) & 2180 & 2193 & $0.59 \%$ \\
\hline Propellant Mass (kg) & 16780 & 16972 & $1.14 \%$ \\
\hline Prop. Mass Fraction & 0.885 & 0.878 & $-0.75 \%$ \\
\hline \multicolumn{5}{|c|}{ Delta IV } \\
\hline Gross Mass (kg) & 30710 & 30564 & $-1.63 \%$ \\
\hline Inert Mass (kg) & 3490 & 3507 & $0.49 \%$ \\
\hline Propellant Mass (kg) & 27200 & 26756 & $-0.48 \%$ \\
\hline Prop. Mass Fraction & 0.89 & 0.875 & $-1.67 \%$ \\
\hline
\end{tabular}


Currently, the main limitation of SPSP is the inability to model crewed vehicle habitats or reentry vehicles. Designs of crewed vehicles must begin with an external habitat estimate that is bookmarked in SPSP as an additional payload.

\subsection{Vehicle Sizing and Mass Estimating Assumptions}

Due to the limitations of SPSP and the desire to mimic NASA's method for returning to the moon, the CEV was taken as the Exploration Systems Architecture Study (ESAS) estimate. The lunar version of the CEV re-entry module weighing $9500 \mathrm{~kg}$ was used, but the service module was not used because only a re-entry module was needed for this study ${ }^{5}$. The crew and additional provisions and equipment were assumed to be $130 \mathrm{~kg}$ per crew member, a total of $520 \mathrm{~kg}$. Similarly, the required habitat mass for the lander was $1050 \mathrm{~kg}^{5}$; this mass included all of the living volume for the four-member crew. All of the architectures used the same masses for the CEV and lunar habitat for uniformity.

Other mass estimating assumptions for the various subsystems of each type of vehicle that was modeled are listed in table 2. The assumptions are specific to SPSP and represent the primary inputs. For the in-space propulsion module (PM) and lander, the assumptions are mostly the same for the expendable and reusable versions. The only difference is in the payloads for the in-space stage; the payloads in table 2 are for the expendable versions. For the reusable vehicles, the PM would only have the CEV and crew as payload. The other difference is in recoverability for the avionics sizing; the distinction is related to the amount of redundancy and vehicle 
health monitoring equipment. Other important assumptions listed are the structural inputs, diameter, configuration, vehicle thrust-to-weight ratio, and engine type.

The structure of the reusable in-space propulsion module (PM-R) that used tank replacement modules (TRMs) was initially modeled in Pro-Engineer, shown in figure 9. The CAD model facilitated understanding how the TRM would connect to the in-space stage and the physical interactions. The CAD model was used to calculate approximate dimension restrictions; the diameter of the TRM was limited to $4 \mathrm{~m}$ as a maximum to prevent the central truss structure within the PM-R from being too large. The larger diameter of $5 \mathrm{~m}$ was used for the PTM because of the assumption that it would be direct heritage from the PM-E. The PM-R uses the $5 \mathrm{~m}$ diameter to accommodate docking to the heat shield side of the CEV; whereas, the lander needed a larger diameter to allow room for the diamond configuration of the four propellant tanks, which was necessary to decrease the height from the habitat to the lunar surface. 
Table 2: Vehicle sizing and mass estimating assumptions.

\begin{tabular}{|c|c|c|c|c|}
\hline & PM & Lander & TRM & PTM \\
\hline \multicolumn{5}{|l|}{ Top Level Assumptions } \\
\hline Diameter $(\mathrm{m})$ & 5 & 7.5 & 4 & 5 \\
\hline Max g-loading (Earth g's) & 3 & 3 & 3 & 3 \\
\hline Active Mission Duration (days) & 8 & 7 & 4 & 4 \\
\hline Configuration & Tandem: Fuel on top & 4 Tanks & Nested & Nested \\
\hline$\Delta \mathrm{V} 1(\mathrm{~m} / \mathrm{s})$ & 4650 & 2445 & $\mathrm{~N} / \mathrm{A}$ & $\mathrm{N} / \mathrm{A}$ \\
\hline$\Delta \mathrm{V} 2(\mathrm{~m} / \mathrm{s})$ & 4555 & 2415 & $\mathrm{~N} / \mathrm{A}$ & $\mathrm{N} / \mathrm{A}$ \\
\hline Payload 1 & CEV+Crew+Lander & Crew & $\mathrm{N} / \mathrm{A}$ & $\mathrm{N} / \mathrm{A}$ \\
\hline Payload 2 & CEV+Crew & Crew & $\mathrm{N} / \mathrm{A}$ & $\mathrm{N} / \mathrm{A}$ \\
\hline Time Between Maneuvers (hrs) & 288 & 168 & $\mathrm{~N} / \mathrm{A}$ & $\mathrm{N} / \mathrm{A}$ \\
\hline \multicolumn{5}{|l|}{ Engine } \\
\hline Vehicle T/W (Earth g's) & $>0.15$ & $>0.17$ & $\mathrm{~N} / \mathrm{A}$ & $\mathrm{N} / \mathrm{A}$ \\
\hline Engine Type & RL-10B-2 & RL-10A-3 & $\mathrm{N} / \mathrm{A}$ & $\mathrm{N} / \mathrm{A}$ \\
\hline \multicolumn{5}{|l|}{ Main Tanks } \\
\hline Axial Tank Buffers (m) & 0.15 & 0.15 & 0.05 & 0.15 \\
\hline Side Tank Buffers $(\mathrm{m})$ & 0.05 & 0.15 & 0 & 0.05 \\
\hline Tank Ullage ( $\%$ of volume) & 5 & 5 & 5 & 5 \\
\hline Operating Pressure (psi) & 40 & 40 & 40 & 40 \\
\hline Factor of Safety & 1.5 & 1.5 & 1.5 & 1.5 \\
\hline Material Type & Graphite Composite & Graphite Composite & Graphite Composite & Graphite Composite \\
\hline Insulation & Goldized MLI & Goldized MLI & Goldized MLI & Goldized MLI \\
\hline Insulation Thickness (mm) & LH2: 50.8/LOX: 25.4 & LH2: 50.8/LOX: 25.4 & LH2: 50.8/LOX: 25.4 & LH2: 50.8/LOX: 25.4 \\
\hline \multicolumn{5}{|l|}{ Propellant Feed } \\
\hline Fuel Lines & 1 & 2 & 1 & 1 \\
\hline Fuel Pressurant Tanks & 1 & 2 & 1 & 1 \\
\hline Fuel Pressurization Method & Autogenous & Autogenous & Autogenous & Autogenous \\
\hline Oxidizer Lines & 1 & 2 & 1 & 1 \\
\hline Oxidizer Pressurant Tanks & 1 & 2 & 1 & 1 \\
\hline Oxidizer Pressurization Method & Cryo Stored $\mathrm{He}$ & Cryo Stored $\mathrm{He}$ & Cryo Stored $\mathrm{He}$ & Cryo Stored He \\
\hline \multicolumn{5}{|l|}{ Reaction Control System } \\
\hline RCS Type & Main Tank Tap-Off & Main Tank Tap-Off & Main Tank Tap-Off & Main Tank Tap-Off \\
\hline Equivalent $\Delta \mathrm{V}$ & 100 & 100 & 50 & 50 \\
\hline Thrust per Thruster (N) & 500 & 500 & 250 & 500 \\
\hline Number of Thrusters & 16 & 16 & 8 & 16 \\
\hline \multicolumn{5}{|l|}{ Power } \\
\hline Power Generation Type & Fuel Cell & Fuel Cell & Fuel Cell & Fuel Cell \\
\hline Nominal Voltage (V) & 28 & 28 & 28 & 28 \\
\hline Mean Required Power (W) & 1500 & 5000 & 250 & 250 \\
\hline Power Margin & $25 \%$ & $25 \%$ & $25 \%$ & $25 \%$ \\
\hline Supply Efficiency & $97 \%$ & $97 \%$ & $97 \%$ & $97 \%$ \\
\hline \multicolumn{5}{|l|}{ Structures } \\
\hline Thrust Structure Material & Aluminum 2219 & Aluminum 2219 & Composite & Aluminum 2219 \\
\hline Aft Skirt Material & None & Aluminum 2219 & None & None \\
\hline Aft Compartment Material & Aluminum 2219 & Aluminum 2219 & None & None \\
\hline Intertank Material & Aluminum 2219 & Aluminum 2219 & None & None \\
\hline Forward Skirt Material & None & Aluminum 2219 & None & None \\
\hline Payload Adapter Material & Aluminum 2219 & Aluminum 2219 & Composite & Aluminum 2219 \\
\hline Docking Adapter & Yes & Yes & No & Yes \\
\hline Habitat Mass (kg) & $\mathrm{N} / \mathrm{A}$ & 1050 & $\mathrm{~N} / \mathrm{A}$ & $\mathrm{N} / \mathrm{A}$ \\
\hline \multicolumn{5}{|l|}{ Avionics } \\
\hline Rendezvous \& Docking & Yes & Yes & Yes & Yes \\
\hline Recoverable & Yes/No & Yes/No & No & No \\
\hline Crewed & Yes & Yes & No & No \\
\hline
\end{tabular}




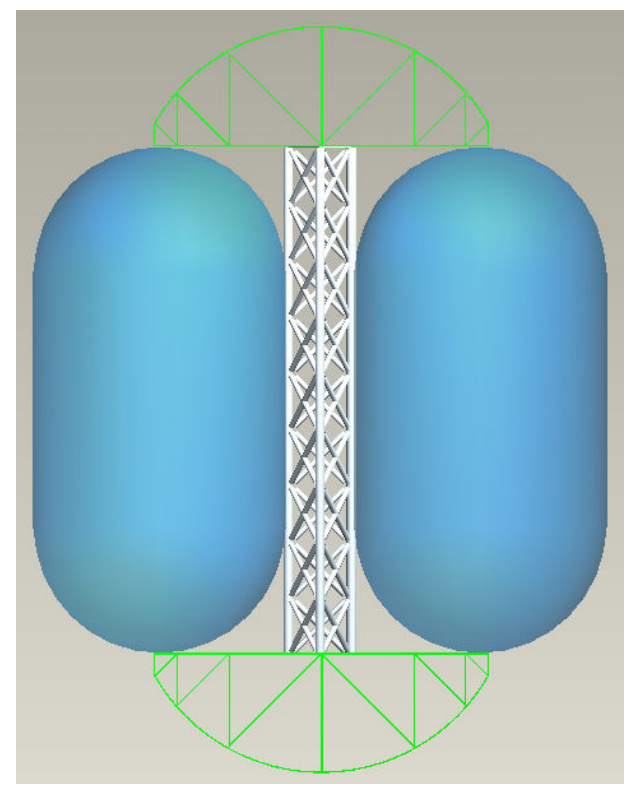

Figure 9: CAD model of PM-R and TRM.

The thrust-to-weight ratios for the propulsive elements, the PM and lander, were selected based on important performance criteria. To minimize gravity losses during large maneuvers, the PM needs to maintain at least 0.15 Earth g's for the vehicle. For the lander, the thrust-to-weight had to be greater than 1 lunar g at lift-off from the lunar surface; this translates to about 0.17 Earth g's. Engines within the RL10 family were selected because of their long history of success and performance. The introduction of commercial off-the-shelf (COTS) engines also removes the engine development costs as a variable in the overall vehicle developments. Engines are not applied to the propellant transfer module (PTM) and TRM because they are non-propulsive elements, only meant for the transportation and delivery of propellant and other fluids and hardware.

The final assumptions for the re-supply scenarios are the amount of propellant transfer loss and additional tank replacement hardware. The propellant transfer loss was calculated as a percentage of the net amount of propellant transferred by the PTM. For example, a transfer loss of $1 \%$ for a net transfer of $1000 \mathrm{~kg}$ would result in 
a $10 \mathrm{~kg}$ loss. Similarly, the additional tank replacement hardware is defined as a percentage of the gross mass of the TRMs. For example, if two TRMs of 500kg each were used on the PM-R, then $1 \%$ of additional hardware would be $10 \mathrm{~kg}$. To establish a baseline for comparison, the propellant transfer loss and additional tank replacement hardware were both assumed to be $0 \%$. A sensitivity analysis was conducted to determine the effects of introducing inefficiencies into the respective re-supply methods, see sections 4.4.1 and 4.4.2.

\section{$\underline{3.3 \text { Velocity Change Maneuvers }}$}

The in-space propulsion module (PM) described in the $\Delta \mathrm{Vs}$ is generic. For the no re-supply scenarios, the PM is expendable; whereas, for the re-supply scenarios it is reusable. The distinction is not made here because of the maneuvers are, for the most part, identical. The differences are explained in sections 2.1.2 and 2.2.3.

The $\Delta$ Vs were calculated based on fundamental orbital mechanics, assuming impulsive maneuvers. Beginning in LEO in a $400 \mathrm{~km}$ circular orbit with an inclination of $28.5^{\circ}$, the TLI is performed to enter a four-day lunar-bound trajectory. Due to pointing errors, engine performance variation, finite maneuvering, and other errors, a trajectory correction maneuver (TCM) may be required, while en route to the moon. In the vicinity of the moon, the LOI is performed to capture the vehicles into a circular $100 \mathrm{~km}$ polar orbit; this orbit allows for global access of the lunar surface, regular communication between the loitering assets and lander, and easier phasing for ascent since the crew visits a nearly polar landing site. During descent, the lander performs up to a twenty-degree plane change to facilitate longer surface durations. 
While the crew is on the lunar surface, the in-space propulsion module with the CEV must maintain the low polar orbit remotely. After the lander ascends, it completes a plane change as necessary, and then completes a rendezvous and docking with the CEV. For redundancy, the PM can remotely perform the plane change and rendezvous and docking. After separation from the lander, the in-space propulsion module (PM) performs the TEI, which includes up to a ninety-degree plane change to allow for a rapid return. The large plane change capability prevents the crew from having to wait in LLO for the appropriate phasing of the Earth and moon. If necessary, a TCM is performed during the four-day coast back to Earth. The last propulsive maneuver is the EOC, which returns the CEV to the initial $400 \mathrm{~km}$ orbit. All of the $\Delta \mathrm{V}$ requirements in meters per second for the Orbital Maneuvering System (OMS) and Reaction Control System (RCS) of each corresponding vehicle are listed in table 3. The RCS $\Delta \mathrm{V}$ requirements were selected arbitrarily to provide adequate margin.

Table 3: Orbital maneuver $\Delta \mathrm{V}$ requirements.

\begin{tabular}{|l|c|c|c|}
\hline & OMS $(\mathrm{m} / \mathrm{s})$ & RCS $(\mathrm{m} / \mathrm{s})$ & Vehicle Type \\
\hline TLI & 3110 & 15 & PM \\
\hline TCM & 20 & 5 & PM \\
\hline LOI & 920 & 10 & PM \\
\hline Descent & 1875 & 25 & Lander \\
\hline Descent plane change & 570 & 25 & Lander \\
\hline Ascent & 1830 & 25 & Lander \\
\hline Plane change & 570 & 10 & Both \\
\hline LLO station keeping & 30 & 10 & PM \\
\hline Rendezvous \& Dock & 15 & 15 & Both \\
\hline TEl & 1410 & 15 & PM \\
\hline TCM & 20 & 5 & PM \\
\hline EOC & 3110 & 15 & PM \\
\hline
\end{tabular}

Since many of the architecture scenarios require that PMs only execute the maneuvers from LEO to LLO or vice versa, the Lunar-bound and the Earth-bound 
$\Delta \mathrm{Vs}$ are listed in table 4 . The point to distinguish between phases of the mission is arbitrary to provide similar $\Delta \mathrm{V}$ totals. Similar $\Delta \mathrm{V}$ totals limit the inefficiency of PMs with only partially full tanks.

Table 4: Lunar-bound and Earth-bound velocity change totals.

\begin{tabular}{|c|c|c|c|}
\hline & OMS $(\mathrm{m} / \mathrm{s})$ & RCS $(\mathrm{m} / \mathrm{s})$ & Orbit \\
\hline Lunar-bound & 4650 & 5 & LEO to Plane change \\
\hline Earth-bound & 4555 & 15 & Rendezvous \& Docking to EOC \\
\hline
\end{tabular}

The Lunar-bound portion of the mission is from LEO to LLO, station keeping, and plane change. To accommodate this separation between the two phases of the mission, the PM performs the orbit boost and plane change remotely, while the crew is on the lunar surface. This puts the PM in the appropriate inclination orbit to rendezvous and dock with the lander. When the PM-E is delivering re-supply modules to LLO, the vehicles are delivered to the same twenty-degrees from polar orbit.

\section{$\underline{3.4 \text { Cost Analysis }}$}

The cost analysis was completed using the "Handbook of Cost Engineering for Space Transportation Systems with Transcost 7.1". Transcost uses regression analysis through historical data to relate cost of a vehicle to mass, vehicle complexity, programmatic experience, development schedule, and other similar qualities. The regressions are separated into two basic categories: engines and vehicles. The engines covered by the model are solid rocket motors, pump-fed liquid rockets, pressure-fed liquid rockets, and air breathing turbo- and ramjet-engines. The 9 types of vehicles modeled are as follows: large solid rocket boosters, liquid propulsive modules, expendable ballistic rocket vehicles, reusable launch vehicles, winged 
orbital vehicles, horizontal take-off first stage vehicles and advanced aircraft, vertical take-off first stage fly-back rocket vehicles, crewed ballistic re-entry capsules, and other crewed space systems. Though Transcost was originally developed as a cost model for launch vehicles, the similarity of in-space propulsion stages has caused the latest versions of Transcost to extend the applications into space vehicles. The model facilitates the estimation of non-recurring, recurring, and operations costs. The nonrecurring costs include detailed system design through building of test models and prototype flight units. Recurring costs encompass materials, processing, assembly, verification tests, acceptance tests, engineering support, and quality assurance costs. The non-recurring costs are higher than the recurring costs by a factor of about 60 times, based on historical data. The operations costs consist of ground preparations of a vehicle, launch and mission operations, administration and management, technical support, and general launch site costs. The operations costs are the most variable and most difficult to accurately predict.

Transcost models use the quantity for cost of Man-Years. A Man-Year is a unit of cost measure independent of time, location, inflation, and currency. The ManYear includes the direct cost of personnel working on a project plus support personnel and overhead costs. The value can be determined as the ratio of total industry project cost to the number of fully accounting people ${ }^{8}$. The average Man-Year value in 2003 was about $\$ 226,400$ in U.S. dollars, assuming 3\% inflation per year the value would be about $\$ 240,000$ in 2005 . However, for simplicity, only the Man-Year costs are considered since only the relative costs of the re-supply methods are of interest. 
The advantage of using Transcost is the ease with which the models could be entered into a series of MATLAB scripts for rapid execution (see appendix). Also, since the models are based primarily on the vehicle masses and similar quantitative information, the number of qualitative user inputs is limited. The primary factors that are subject to interpretation are the production learning curve factor, team experience factor, and technology development factor. The learning curve factor dictates the amount of reduced cost per element for each additional element of the same type that is produced. The learning curve factor is related to the element size and production totals; as the mass of an element increases or the production total decreases, the learning curve factor approaches a value of one. The experience factor is the amount of applicable team experience as applied to the development of the particular vehicle, and the development factor is the relative comparison of the new vehicle to existing projects and technologies. The experience and development factors are both normalized to a scale from zero to ten. For the experience factor, a value of zero indicates the highest level of applicable team experience, and a development factor value of zero is the least variation from existing projects and technologies.

The three qualitative factors are listed in table 5 for each element in each architecture type. The learning curve factor is smaller for the two re-supply scenarios because the elements require greater production rates and/or the elements are smaller than the no re-supply elements. The experience factor was selected as five to assume that the team involved has some related experience for the development and production of the PMs and similar elements. The landers have a larger experience factor because it has been 40 years since the last crewed lunar lander was developed. 
The development factors were applied to elements as the use of new technologies. For PM-Es, the development factor was selected to indicate a limited application of new technologies. The propellant transfer PM-R required a higher value for the development factor because of the application of the completely new propellant transfer system to otherwise modern designs. For propellant transfer, the burden of transferring the propellant to the receiving tank almost entirely falls upon the resupply vehicle, i.e. the PTM. Because of this added requirement to the PTM and not the PM-R, the development factor was higher for the PTM than the PM-R. However, the burden of re-supply is higher on the PM-R and TRM for the replacement tanks method, as compared to their counterparts in propellant transfer because of the redesign of the structure and integration of the two elements. This leads to a higher development factor for the tank replacement PM-R and TRM over their counterparts in the propellant transfer scenarios. Similar logic was applied in the selection of the development factors for the remaining elements. These initial values of the qualitative factors form the cost analysis baseline.

Table 5: Transcost assumptions for the qualitative factors.

\begin{tabular}{|c|c|c|c|}
\hline Mission Element & Learning Factor & Experience Factor & Development Factor \\
\hline No Re-supply & & & \\
\hline Lander & 0.9 & 8 & 8 \\
\hline PM-E & 0.9 & 5 & 6 \\
\hline Propellant Transfer & & & 6 \\
\hline PM-E & 0.85 & 5 & 7 \\
\hline PM-R & 0.85 & 5 & 8 \\
\hline Lander & 0.85 & 8 & 8 \\
\hline PTM & 0.85 & 5 & 6 \\
\hline Replacement Tanks & & & 8 \\
\hline PM-E & 0.85 & 5 & 8 \\
\hline PM-R & 0.85 & 5 & 9 \\
\hline Lander & 0.85 & 8 & \\
\hline TRM & 0.85 & 5 & \\
\hline
\end{tabular}




\subsection{Launch Vehicles}

To complete the analysis, the launch vehicle capabilities are necessary to understand the mission operations, sequencing, and feasibility. The two NASA Shuttle-Derived Launch Vehicles (SDLVs) from the Exploration Systems Architecture Study (ESAS) were selected to ground the analysis in reality. In each of the architectures, the manned launch vehicle is dedicated to only launching the CEV, and the unmanned launch vehicle has an assumed capability of lifting 130 metric tons of cargo. Both SDLVs are able to deliver their respective payloads to a $400 \mathrm{~km}$ circular orbit at about $28.5^{\circ}$ inclination. The capabilities used for this study may differ slightly from the actual SDLVs because this study commenced prior to the public release of the SDLV configurations and capabilities.

The cost for the development of the SDLVs is not calculated here. Since all of the architectures use the exact same launch vehicles, the development cost of the SDLVs would not cause a change in the relative expenses. The development costs would simply increase all of the cost curves by the same amount.

The launch vehicle production and operations costs were ignored for this study. Based on the Shuttle Transportation System (STS), the fixed costs are assumed to be much more significant than the variable costs; this is largely due to the many thousands of personnel and large infrastructure that must be maintained. The SDLVs will be more expendable in comparison to STS; however, the invariable program costs are assumed to outweigh the variable per launch costs. 


\subsection{Analysis Procedure Automation}

To facilitate rapid analysis of each architecture option, the procedure of sizing element masses and performing cost analyses was automated in two steps. First, the SPSP workbooks for each element were created with the appropriate settings for each of the architectures and linked to the other necessary element workbooks. All of the data was collected into one central workbook, allowing the architecture elements to be sized for each scenario variation. Next, a series of MATLAB scripts (see appendix) were created to collect the data from the Microsoft Excel workbooks, execute the Transcost scripts to perform the cost analysis, record the data in another centralized workbook, and generate graphical outputs of the cost results. The automated procedure offers the rapid variation of parameters to understand the sensitivities of the results to specific inputs. The time to perform the analysis of a single architecture was reduced to about 30 minutes for the mass sizing and less than 5 or 10 minutes for the cost analysis. Due to the time intensive nature of the mass sizing procedure, variations in the mass estimating assumptions could lead to longer analysis times. However, prior to the automation of both parts of the analysis, a single design point analysis from beginning to end took nearly half of a workday. The manual procedure was drawn out because of the time to check for errors and discrepancies for each iteration. Error checking was not made a direct part of the automated procedure; but after an analysis was performed, errors only had to be checked once before continuing.

One of the scripts developed (see appendix) calculates the number of each type of element needed to complete a mission. The algorithm determines the number 
of missions left for any reusable element and the number of expendable elements that need to be delivered during the mission. The script was developed to determine quickly the total number of production elements necessary for a given total number of missions and a predetermined reusability; the reusability of an element is the total number of missions that element can be used. 


\section{Chapter 4: Results and Comparisons}

\subsection{Mass Estimates}

The mass estimates discussed in this section are the direct output of SPSP. The detailed subsystem mass breakdown and geometry sizing were omitted since only the top-level mass breakdown was used for the cost analysis.

\subsubsection{No Re-supply}

The mass estimating results for the no re-supply architectures are shown in table 6. The gross masses do not include any payload masses, and the propellant mass listed is only the usable propellant mass. The inert masses include all dry mass plus residual propellants, pressurant and purge gases, and reaction control system (RCS) propellants. As explained in section 2.1.2, the NR-2 scenario requires two identical expendable in-space propulsion modules (PM-Es) per mission, one for the lunar-bound portion and one for the earth-bound portion of the mission; the NR-3 does not include an Earth orbit capture (EOC), but a direct entry, descent, and landing (EDL) is performed instead.

Table 6: No re-supply mass estimates.

\begin{tabular}{|c|c|c|c|c|}
\hline & Scenario & NR-1 & NR-2 & NR-3 \\
\hline CEV+Crew & Gross $(\mathrm{kg})$ & 10020 & 10020 & 10020 \\
\hline & & & & \\
\hline \multirow{4}{*}{ PM-E } & Gross $(\mathrm{kg})$ & 273490 & 89732 & 102024 \\
\cline { 2 - 5 } & Propellant $(\mathrm{kg})$ & 250204 & 80731 & 90571 \\
\cline { 2 - 5 } & Inert $(\mathrm{kg})$ & 23286 & 9001 & 11453 \\
\cline { 2 - 5 } & Dry $(\mathrm{kg})$ & 12017 & 6559 & 7092 \\
\hline \multirow{4}{*}{ Lander } & & & & \\
\cline { 2 - 5 } & Gross $(\mathrm{kg})$ & 16643 & 16643 & 16643 \\
\cline { 2 - 5 } & Propellant $(\mathrm{kg})$ & 11466 & 11466 & 11466 \\
\cline { 2 - 5 } & Inert $(\mathrm{kg})$ & 5177 & 5177 & 5177 \\
\cline { 2 - 5 } & Dry $(\mathrm{kg})$ & 4398 & 4398 & 4398 \\
\hline
\end{tabular}


Due to the unmanned launch vehicle payload capability of 130 tons to LEO, the NR-1 scenario is not feasible even if all elements are launched separately. NR-2 must be completed in at least three launches: one for crew and two for cargo. NR-3 could be completed in a single launch; however, crew is prohibited from launching with cargo. Therefore, NR-3 requires a minimum of two launches: one for crew and one for cargo every mission.

\subsubsection{Propellant Transfer}

The various masses for the propellant transfer scenarios are shown in table 7 . The scenario naming convention is based on the percentage of propellant transfer loss; for example, PT-50 stands for $50 \%$ transfer loss, where the loss is a percentage of the total propellant on the propellant transfer module (PTM). For easier comparison, some of the various propellant transfer losses that are considered in section 4.4.1 are also in table 7. The transfer loss is carried as unusable propellant in the inert mass of the PTM. The PTM propellant mass is the amount of propellant that would actually be transferred. The PM-E was sized to deliver one lander and one PTM to LLO. This drove the PTM to be the same gross mass as the lander in all propellant transfer scenarios; by making them the same gross mass, the PM-E either delivers a full lander and one PTM or two PTMs. This provided the capability to deliver a lander with the necessary PTM for refilling it on the next mission, see section 2.2.3 for a description of the advantages to be gained.

For the first mission in a campaign for a reusable in-space propulsion module (PM-R) and a reusable lander, all of the propellant transfer scenarios require one 
manned launch vehicle and two unmanned launch vehicles. Based only on the vehicle masses, the PM-R could launch with two PTMs and the lander; the second cargo launch is necessary for the PM-E. However, the possibility exists that the combined heights of the PTMs, lander, and PM-R would exceed the launch vehicle shroud allotment. Therefore, it is likely that a total of three unmanned launch vehicles are required. For the missions that re-supply the PM-R and lander, two cargo launches are required in addition to the crew launch. However, before any of the subsequent missions in the campaign are attempted, a cargo launch with two PTMs is required to re-supply the PM-R that is loitering in LEO.

Table 7: Propellant transfer mass estimates.

\begin{tabular}{|c|c|c|c|c|c|c|}
\hline & Scenario & PT-0 & PT-2 & PT-4 & PT-15 & PT-50 \\
\hline CEV+Crew & Gross (kg) & 10020 & 10020 & 10020 & 10020 & 10020 \\
\hline \multirow{4}{*}{ PM-E } & Gross (kg) & 118107 & 118107 & 118107 & 118107 & 118107 \\
\hline & Propellant $(\mathrm{kg})$ & 75748 & 75748 & 75748 & 75748 & 75748 \\
\hline & Inert $(\mathrm{kg})$ & 9073 & 9073 & 9073 & 9073 & 9073 \\
\hline & Dry $(\mathrm{kg})$ & 6192 & 6192 & 6192 & 6192 & 6192 \\
\hline & & & & & & \\
\hline \multirow{4}{*}{ Lander } & Gross (kg) & 16643 & 16643 & 16643 & 16643 & 16643 \\
\hline & Propellant (kg) & 11466 & 11466 & 11466 & 11466 & 11466 \\
\hline & Inert $(\mathrm{kg})$ & 5177 & 5177 & 5177 & 5177 & 5177 \\
\hline & Dry (kg) & 4398 & 4398 & 4398 & 4398 & 4398 \\
\hline & & & & & & \\
\hline \multirow{4}{*}{ PM-R } & Gross (kg) & 43580 & 43580 & 43580 & 43580 & 43580 \\
\hline & Propellant $(\mathrm{kg})$ & 27950 & 27950 & 27950 & 27950 & 27950 \\
\hline & Inert $(\mathrm{kg})$ & 5607 & 5607 & 5607 & 5607 & 5607 \\
\hline & Dry (kg) & 4439 & 4439 & 4439 & 4439 & 4439 \\
\hline & & & & & & \\
\hline \multirow{6}{*}{ PTM } & Gross (kg) & 16643 & 16643 & 16643 & 16643 & 16643 \\
\hline & Propellant $(\mathrm{kg})$ & 14632 & 14353 & 14084 & 12451 & 7412 \\
\hline & Inert $(\mathrm{kg})$ & 2011 & 2290 & 2559 & 4192 & 9231 \\
\hline & Dry (kg) & 1511 & 1511 & 1511 & 1512 & 1524 \\
\hline & \# for PM-R & 1.91 & 1.95 & 1.98 & 2.24 & 3.77 \\
\hline & \# for Lander & 1.02 & 1.04 & 1.06 & 1.19 & 2.00 \\
\hline
\end{tabular}


In table 7, the number of PTMs that are required to re-supply the PM-R and lander are listed. A fraction of a PTM implies that the remaining PTM propellant load is considered available for the next mission, thereby reducing the PTM demand for the next mission. This was considered since only whole numbers of fully loaded PTMs could be launched and delivered. For PT-0, this shows that only 3 PTMs must be used since the total number required is 2.93; for the calculation of the number of PTMs for the next mission, the extra 7\% of the PTM is carried over. This provides added capability of the system to exceed the minimum number of required PTMs. As seen in section 4.2.2, this results in small differences in production quantities for different transfer losses.

Since the gross masses of the PM-R and lander are the same for all amounts of propellant transfer loss, only the deliverable PTM propellant mass changes, which changes the number of required PTMs. The change to PTM deliverable propellant mass is inversely proportional to the change in transfer loss. For example, as compared to $0 \%$ transfer loss, an increase to $2 \%$ reduces the available propellant in the PTM by $1.9 \%$; an increase to $4 \%$ causes the available propellant to drop by $3.7 \%$; an increase to $15 \%$ reduces the PTM transferable propellant by $14.9 \%$; and an increase to $50 \%$ transfer loss cuts the available PTM propellant by $49.3 \%$.

Similarly, the change in the required number of PTMs is directly proportional to the change in transfer loss. A $2 \%$ increase in transfer loss, yields an increase in the number of required PTMs by about $2 \%$, and an increase to $4 \%$ transfer loss only increases the number of required PTMs by 3.8\%. However, an increase from $0 \%$ to $15 \%$ transfer loss causes the number of necessary PTMs to increase by over $17 \%$. 
The 50\% transfer loss case doubles the number of PTMs required for $0 \%$ transfer loss. Since the propellant transfer scenarios were sized assuming two PTMs are delivered to LLO by one PM-E, a large increase in propellant transfer loss could drastically affect the operations because of the added PTMs and PM-Es. Also, significant increases in the number of PM-Es would likely drive up the costs per mission.

\subsubsection{Tank Replacement}

Similar to the naming system for the propellant transfer scenarios, the tank replacement scenarios are named based on the additional tank replacement hardware as a percentage of the total mass of all tank replacement modules (TRMs) attaching to the reusable vehicles; the selection of the tank replacement hardware percentages is explained in section 4.4.2. For the PM-R, two TRMs are required; whereas, the reusable lander only requires a single TRM. Even though the lander was designed to use one TRM, the propellant load within one TRM was, in most cases, greater than the actual amount of propellant needed by the lander. The TRM was assumed to be delivered full, and the lander would only use the necessary amount of propellant. Unlike the propellant transfer scenario, any unused portion of propellant was discarded with the TRM. The change in philosophy for tank replacements is based on the operations: the TRM is integrated into the vehicle it re-supplies. Since a particular number of TRMs would re-supply a reusable vehicle, unused portions of the previous TRM could not be used. 
In table 8 , the mass estimates of each element for the tank replacement scenarios are shown. For the PM-R and lander, the gross mass includes the TRMs, the propellant mass is the amount of propellant supplied by the TRMs, the inert mass includes the inert mass of the TRMs, but the dry mass does not include the TRMs.

The PM-R dry mass increases when the percentage of additional hardware increases, which causes required propellant load and TRM gross mass to increase. As the TRM mass increases, the PM-R plus TRM inert mass increases, thus further increasing the required propellant. The effects are propagated through the other architecture elements. Due to this complex coupling of the elements, linear increases in the percentage of additional hardware result in non-linear increases in element gross masses. The TRM gross mass increase varies from $2 \%$ for a $1 \%$ increase in additional hardware to $69.5 \%$ for a $20 \%$ increase; the percentage of gross mass increase for the TRM is 2.1 to 3.5 times the percentage of additional hardware. Similarly, the PM-R gross mass increases from 2.9 to 4.8 times the percentage of tank replacement hardware, and the lander gross mass increase is from 3.2 to 6.9 times. The PM-E exhibits a different characteristic because for $20 \%$ additional hardware and above the PM-E payload is only one TRM or lander. As a result, the PM-E gross mass increase is from 2.6 to 3.6 for $1 \%$ hardware to $15 \%$, and the gross mass increase is only 0.66 times for $20 \%$ hardware and above. However, this deviation from the expected behavior is at the expense of doubling the required number of PM-Es. 
Table 8: Tank replacement mass estimates.

\begin{tabular}{|c|c|c|c|c|c|c|}
\hline & Scenario & TR-0 & TR-1 & TR-2 & TR-10 & TR-20 \\
\hline CEV+Crew & Gross (kg) & 10020 & 10020 & 10020 & 10020 & 10020 \\
\hline \multirow{4}{*}{ PM-E } & Gross $(\mathrm{kg})$ & 72212 & 74069 & 76060 & 98166 & 81741 \\
\hline & Propellant $(\mathrm{kg})$ & 63963 & 65697 & 67558 & 87752 & 72868 \\
\hline & Inert $(\mathrm{kg})$ & 8249 & 8371 & 8502 & 10414 & 8873 \\
\hline & Dry (kg) & 5783 & 5844 & 5909 & 7102 & 6093 \\
\hline & & & & & & \\
\hline \multirow{4}{*}{ Lander } & Gross (kg) & 13355 & 13783 & 14238 & 18928 & 31475 \\
\hline & Propellant $(\mathrm{kg})$ & 9254 & 9542 & 9848 & 13003 & 21444 \\
\hline & Inert (kg) & 4101 & 4241 & 4390 & 5925 & 10031 \\
\hline & Dry (kg) & 3808 & 3938 & 4077 & 5506 & 9330 \\
\hline \multirow{4}{*}{ PM-R } & Gross (kq) & 29733 & 30586 & 31475 & 40254 & 58178 \\
\hline & Propellant $(\mathrm{kg})$ & 24862 & \begin{tabular}{|l|}
25402 \\
\end{tabular} & 25964 & 31512 & \begin{tabular}{|l|}
42834 \\
\end{tabular} \\
\hline & Inert (kg) & 4870 & 5184 & 5511 & 8742 & 15344 \\
\hline & Dry (kg) & 2211 & 2492 & 5669 & 11538 & 10894 \\
\hline & & & & & & \\
\hline \multirow{6}{*}{ TRM } & Gross (kg) & 13759 & 14046 & 14344 & 17291 & 23317 \\
\hline & Propellant (kg) & 12431 & 12701 & 12982 & 15756 & 21417 \\
\hline & Inert (kg) & 1328 & 1345 & 1362 & 1535 & 1900 \\
\hline & Dry $(\mathrm{kg})$ & 993 & 1002 & 1011 & 1110 & 1325 \\
\hline & \# for PM-R & 2.00 & 2.00 & 2.00 & 2.00 & 2.00 \\
\hline & \# for Lander & 0.74 & 0.75 & 0.76 & 0.83 & 1.00 \\
\hline
\end{tabular}

For the first mission in a campaign, at least two cargo launches are required. The first would deliver a PM-E and a PM-R to LEO; the second would deliver the lander, three TRMs, and another PM-E. The third TRM is an extra since the PM-Es are sized to deliver two TRMs or a TRM and a lander to LLO. By delivering an extra TRM, inefficiencies of underutilizing the PM-E are avoided and an extra TRM for redundancy and safety is available. If unused, the extra TRM can be left in LLO until the next mission. For every follow-on mission in the campaign, two TRMs must be launched before the mission can begin to re-supply the PM-R in LEO. The number of necessary TRMs is at most two for the PM-R and one for the lander, leaving room to 
bring out an extra, resulting in two cargo launches; each with one PM-E and two TRMs. Also, a separate crew launch is required for all missions.

\subsection{Cost Estimates}

The cost estimates were generated by Transcost in a MATLAB environment for rapid evaluation and analysis. The detailed roll-up costs were not included since only the overall vehicle development and production costs were of interest.

\subsubsection{Development Costs}

The total development cost in Man-Years for each of the elements is listed in table 9. Since the cost model is based on inert mass as the primary evaluator, it is expected that larger vehicles would be more expensive, especially for the same level of team experience and technology. The PM-E for NR-1 and NR-3 demonstrate this. However, the development cost for the PM-E in NR-2 is higher than the counterpart in NR-3 despite the lower inert mass because the development cost includes the added complexity of an additional stage for NR-2. 
Table 9: Development costs for each element in all architectures.

\begin{tabular}{|c|c|c|c|c|c|}
\hline Scenario & PM-E & PM-R & Lander & PTM/TRM & Total Development Cost \\
\hline NR-1 & 12253 & N/A & 42274 & N/A & 54527 \\
\hline NR-2 & 9233 & N/A & 42274 & N/A & 51507 \\
\hline NR-3 & 7890 & N/A & 42274 & N/A & 50164 \\
\hline & & & & & 62811 \\
\hline PT-0 & 8043 & 11363 & 42274 & 1131 & 62811 \\
\hline PT-2 & 8043 & 11363 & 42274 & 1131 & 62811 \\
\hline PT-4 & 8043 & 11363 & 42274 & 1131 & 62812 \\
\hline PT-15 & 8043 & 11363 & 42274 & 1132 & 62817 \\
\hline PT-50 & 8043 & 11363 & 42274 & 1137 & 63602 \\
\hline & & & & & 65731 \\
\hline TR-0 & 7436 & 15183 & 40010 & 973 & 67749 \\
\hline TR-1 & 7530 & 16694 & 40530 & 977 & 82640 \\
\hline TR-2 & 7628 & 18069 & 41070 & 983 & 102683 \\
\hline TR-10 & 8529 & 27030 & 46047 & 1034 & \\
\hline TR-20 & 7900 & 37470 & 56174 & 1140 & \\
\hline
\end{tabular}

The development cost of the PM-E for the tank replacement scenario decreases from TR-10 to TR-20 because the inert mass of the PM-E was decreased for TR-20; as explained in section 4.1.3, the PM-E capability for TR-20 was decreased from two TRMs to one, which reduces the PM-E gross and inert masses. This was necessary so that the PM-E could fit the launch vehicle constraints. Though, the development cost was reduced, the production costs are impacted, as explained in section 4.4.2.

\subsubsection{Production Costs}

The production costs are based on the total number of each type of element that must be produced. For the no re-supply scenarios, the total number of each element is equal to the number of missions to be attempted, except for NR-2 which requires two PM-Es per mission. For example, to attempt 10 missions: NR-1 and NR-3 require 10 PM-Es and 10 landers compared to NR-2 with 20 PM-Es and 10 
landers. Despite NR-1 being infeasible, as explained in section 4.1.1, further analysis was completed for comparison.

The number of necessary elements versus the number of attempted missions for the propellant transfer and tank replacement scenarios is shown in figures 10 and 11, respectively. The number of attempted missions considered was increased to 250 to clearly demonstrate the dissimilarity between the small amounts of inefficiencies in each of the re-supply methods. For the analyses, a reasonable maximum number of missions was not imposed; however, the feasible number of attempted missions is addressed in section 5.1.

The number of PM-Rs and landers are the same because both vehicle types are replaced at the same time. The number of missions for which the lander and PM$\mathrm{R}$ can be used is 5 ; this means on mission $1,6,11$, etc, the reusable elements are replaced. The sensitivity of the number of missions for which a reusable element can be used is evaluated in section 4.4.4.

For the tank replacement scenario, the number of TRMs is the same for all amounts of additional hardware because the demand is always two for the PM-R and one for the lander. Also, the number of PM-Es is only different for the TR-20 scenario because for this scenario the PM-E is sized to only deliver one TRM at a time; hence, the number for TR-20 of PM-Es is exactly double. This is very different than the propellant transfer scenarios. For propellant transfer, a fraction of a PTM could be used, saving the remaining propellant for the next mission, as explained in section 4.1.2. This causes each propellant transfer scenario to have a unique requirement for the number of PTMs, which influences the number of PM-Es. For 
the smaller amounts of propellant transfer loss, the number of PTMs and PM-Es are similar because the amount of propellant delivered by each PTM is not changing significantly. For large amounts of transfer loss, the amount of propellant delivered drastically decreases.
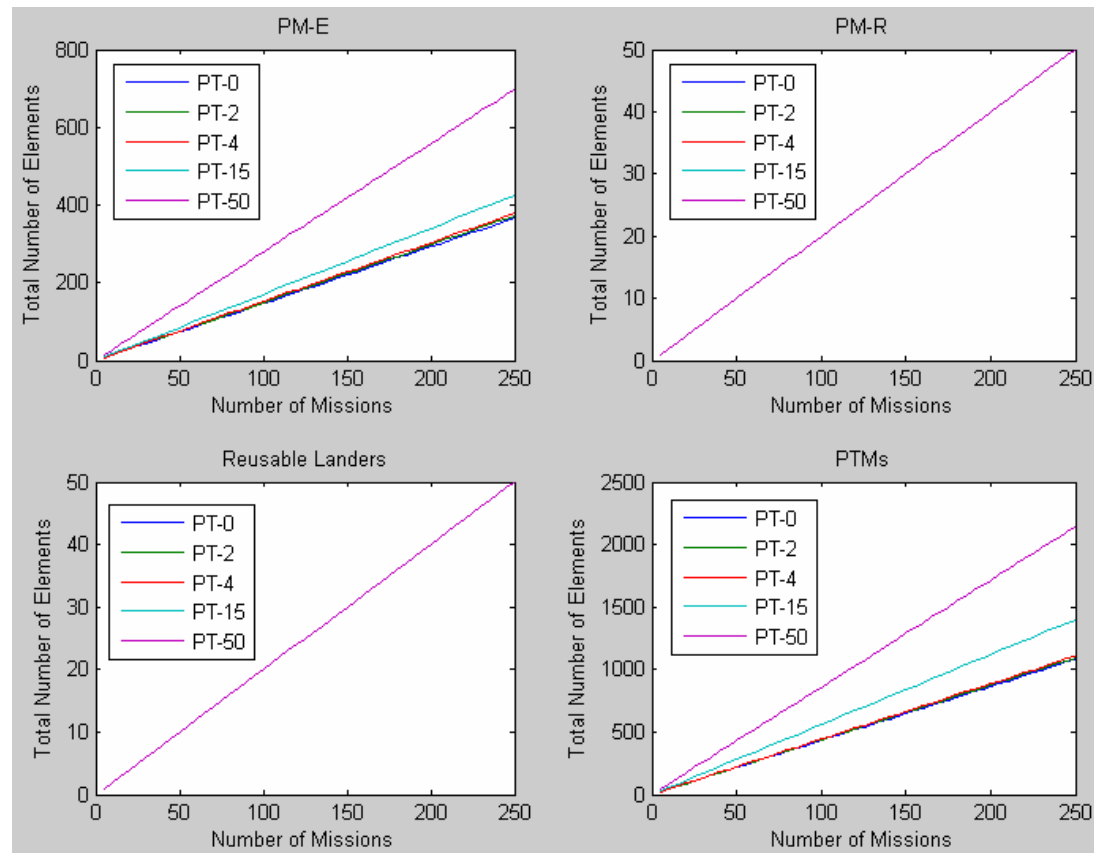

Figure 10: Production numbers for propellant transfer.
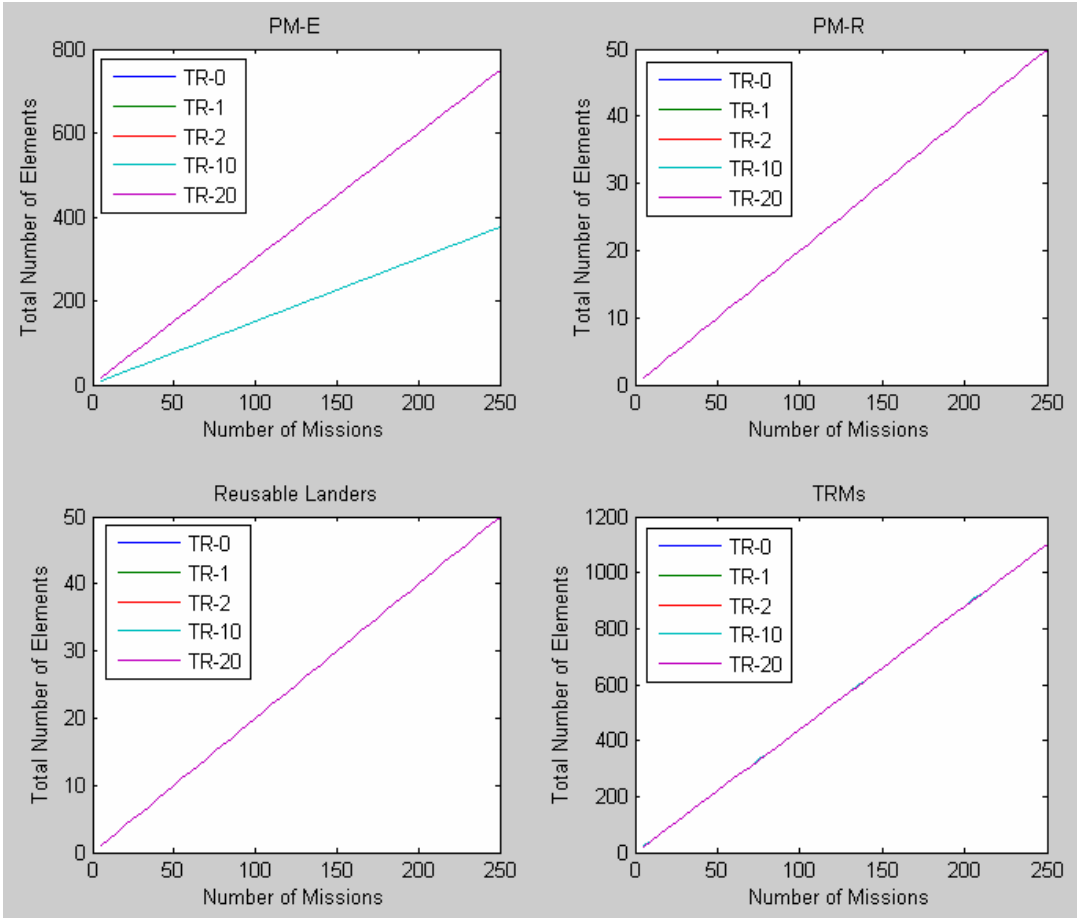

Figure 11: Production numbers for tank replacements. 
For all of the architectures, the total production costs will increase as the number of attempted missions increase, and the average production cost per mission will decrease as more missions are attempted because of the advantages of a production learning curve. On a log-log plot, all of the production cost data exhibits the same behavior: a series of parallel, or nearly parallel, lines. This is demonstrated with the no re-supply architectures in figure 12 . The increase in total production cost of NR-1 and NR-2 over that of NR-3 is $11 \%$ and $30 \%$, respectively. This percentage indicates the increased cost to produce either NR-1 or NR-2. This growth in cost is the same percentage for total production cost and average production cost per mission. Similarly, the growth of the production cost with increased inefficiencies of the two re-supply methods are shown in figure 13; propellant transfer values are plotted on left and tank replacement are on the right. The solid line was generated using intermediate values of the inefficiencies, and the circles are the values of the inefficiencies that were specifically highlighted in sections 4.1.2 and 4.1.3.

For tank replacements, the percentage of increase in production cost over TR0 is about 1.5 to 1 for increases in the percentage of additional tank replacement hardware below $15 \%$; above $15 \%$, the slope of the line becomes much steeper at about 3 to 1 . The ratio becomes much larger as a result of the decrease in payload capability of the PM-E for $20 \%$ and $25 \%$ additional hardware, as described in section 4.2.1.

For propellant transfer, the ratio of percentage of increase in production cost over PT- 0 to percentage of propellant transfer loss has three regions. From $0 \%$ to $4 \%$ transfer loss, the ratio is 0.5 to 1 ; from $4 \%$ to $6 \%$, the ratio is about 1.3 to 1 ; from $6 \%$ 
to $29 \%$, the ratio begins at about 1 to 1 and decreases to about 0.8 to 1 ; and above $29 \%$, the ratio is slightly above 1 to 1 . These regions are the result of changes to the number of production elements as propellant transfer increases. A small increase in transfer loss causes a fractional increase in the number of necessary PTMs, but the minor increase in the number of PTMs results in the need for a whole additional PTM to be launched. An increase of one PTM at launch raises the number of PM-Es by one; and since PM-Es are designed to deliver 2 PTMs, yet another PTM is launched to avoid inefficiencies of underutilized elements. The net result is an increase in the total number of PTMs by two and an increase in PM-Es by one. Only fractions of the two additional PTMs are used, causing the next level of transfer loss to use a larger percentage of the PTMs already being produced without increasing the total number of production elements. This complete picture of the impact of the transfer loss demonstrates that for similar amounts of transfer loss, the number of production elements is similar. However, there are a few transfer loss values such that a small increment in the transfer loss causes a discontinuous jump in the required number of production elements. The change in ratio of percentage of increase in production cost to percentage of propellant transfer loss is a result of this interaction. 

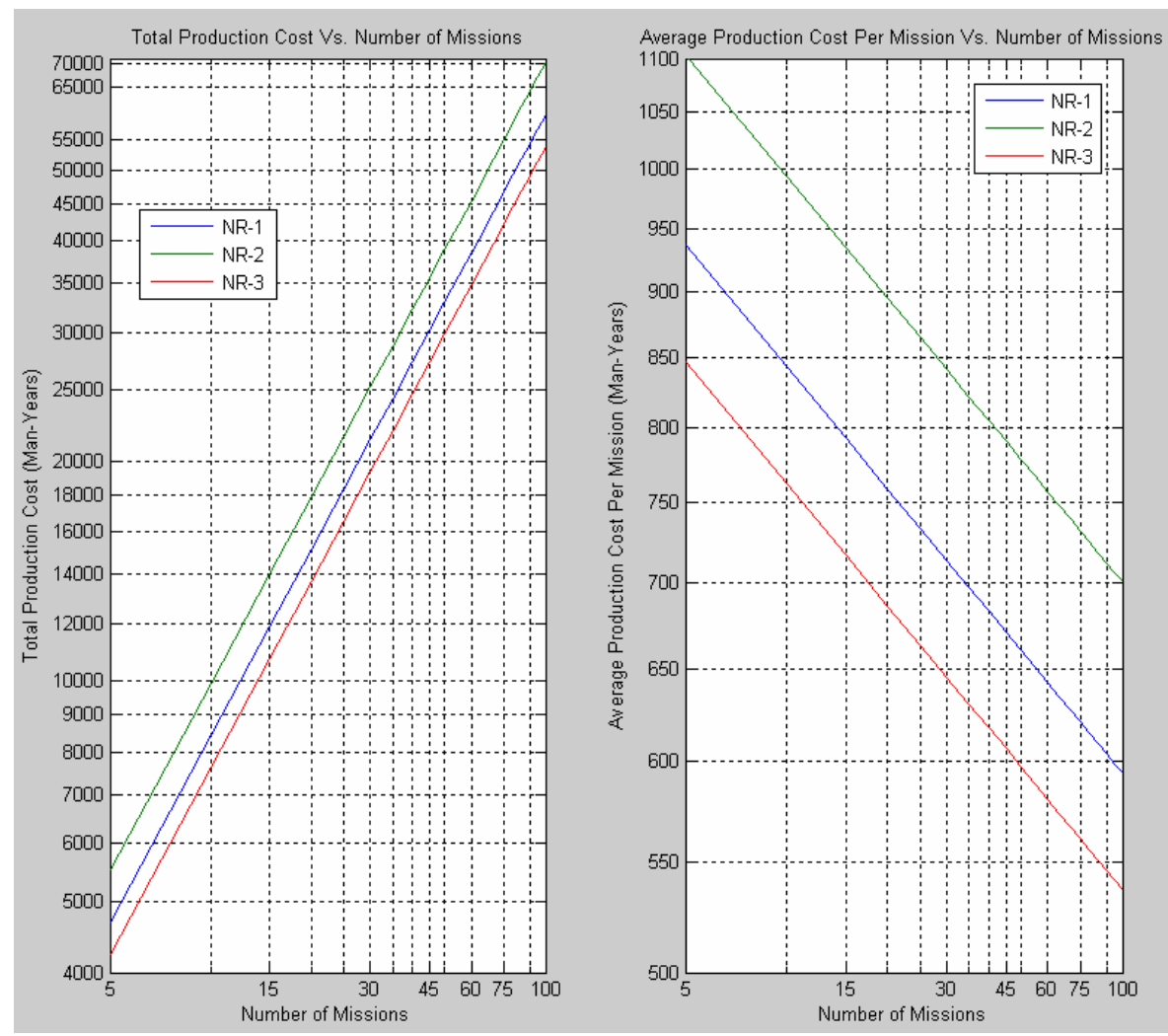

Figure 12: No re-supply total and average production versus number of missions.
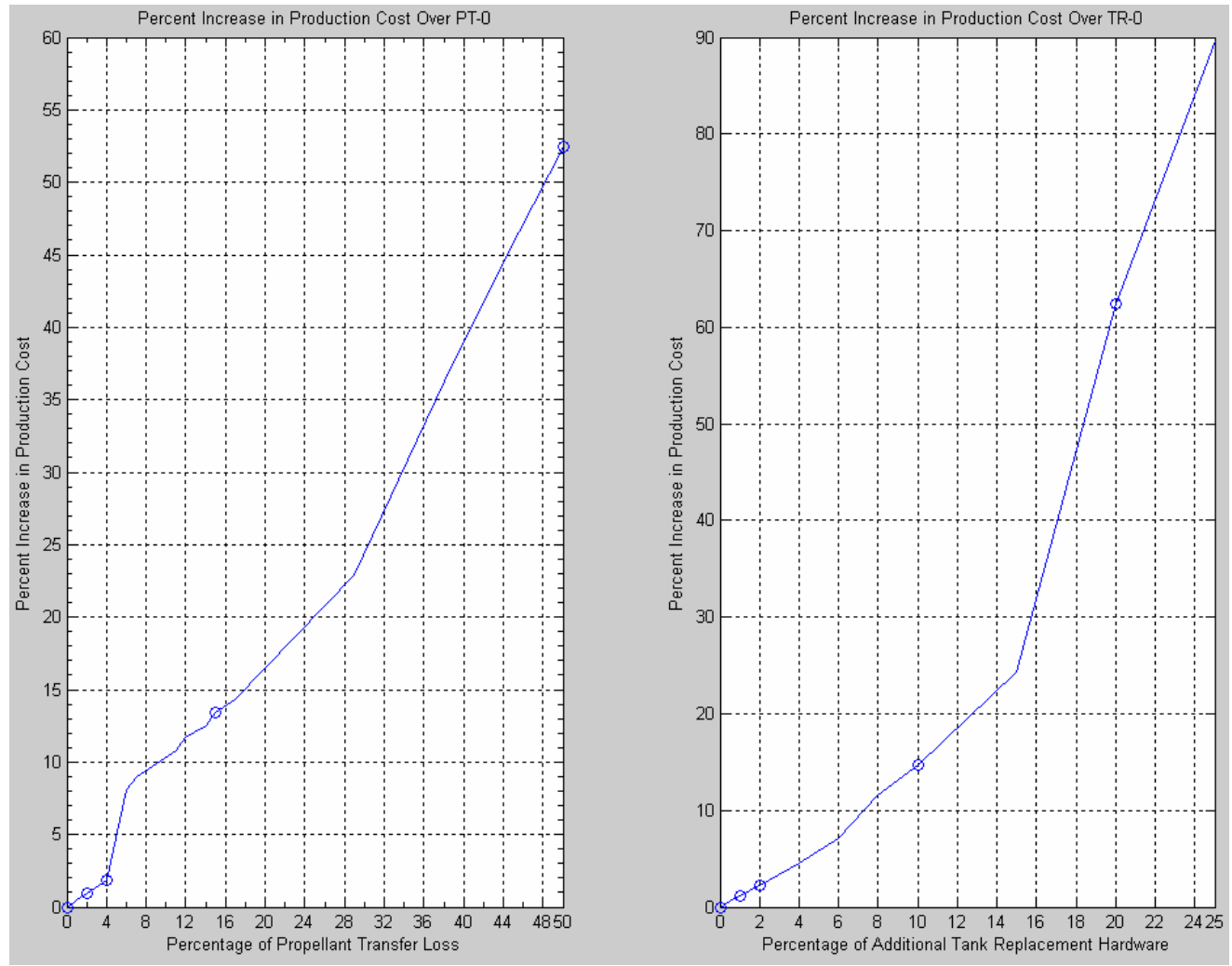

Figure 13: Percent increase in production cost over PT-0 and TR-0. 


\subsection{Comparisons}

The comparisons discussed in this section were completed in three groupings. The no re-supply architectures were compared independently from the re-supply scenarios to determine the best no re-supply scenario, which was then used as a base of reference for other analyses. The re-supply scenarios were compared independent of the no re-supply architectures to facilitate a direct assessment. Lastly, the best no re-supply architecture was compared to both re-supply methods to supplement the independent re-supply comparison.

\subsubsection{No Re-supply Architectures}

The total cost per mission for all of the no re-supply architectures decreases as the number of missions increases, shown in figure 14. The total cost per mission is the total cost for all missions divided by the number of missions that have been attempted; this is the average total cost. The average total cost can also be calculated as the sum of the recurring costs per mission and the amortized non-recurring costs. The amortized non-recurring costs decrease with more missions because the nonrecurring costs are up-front costs that are independent of the number of missions to be attempted. Also, the recurring costs decrease with more missions because the production learning curve reduces the cost per element as more elements are produced, as explained in section 4.2.2.

For 100 missions, the difference between NR-2 and NR-3, the top and bottom lines, respectively, is about 175 Man-Years per mission or about $\$ 42$ million per mission in 2005 dollars. This is about a $17 \%$ savings. However, the savings of $\$ 42$ 
million dollars is applied to every mission for a total difference of $\$ 4.2$ billion. At 5 missions, when NR-2 and NR-3 are at the closest average total cost, NR-2 is only about 5\% more expensive per mission or 525 Man-Years. This translates to $\$ 126$ million per mission for a total of $\$ 630$ million. Similarly, the difference between NR1 and NR-3 grows from $8.8 \%$ to $9.8 \%$; in 2005 dollars, this is a difference of $\$ 1.2$ billion at 5 missions growing to $\$ 2.4$ billion at 100 missions.

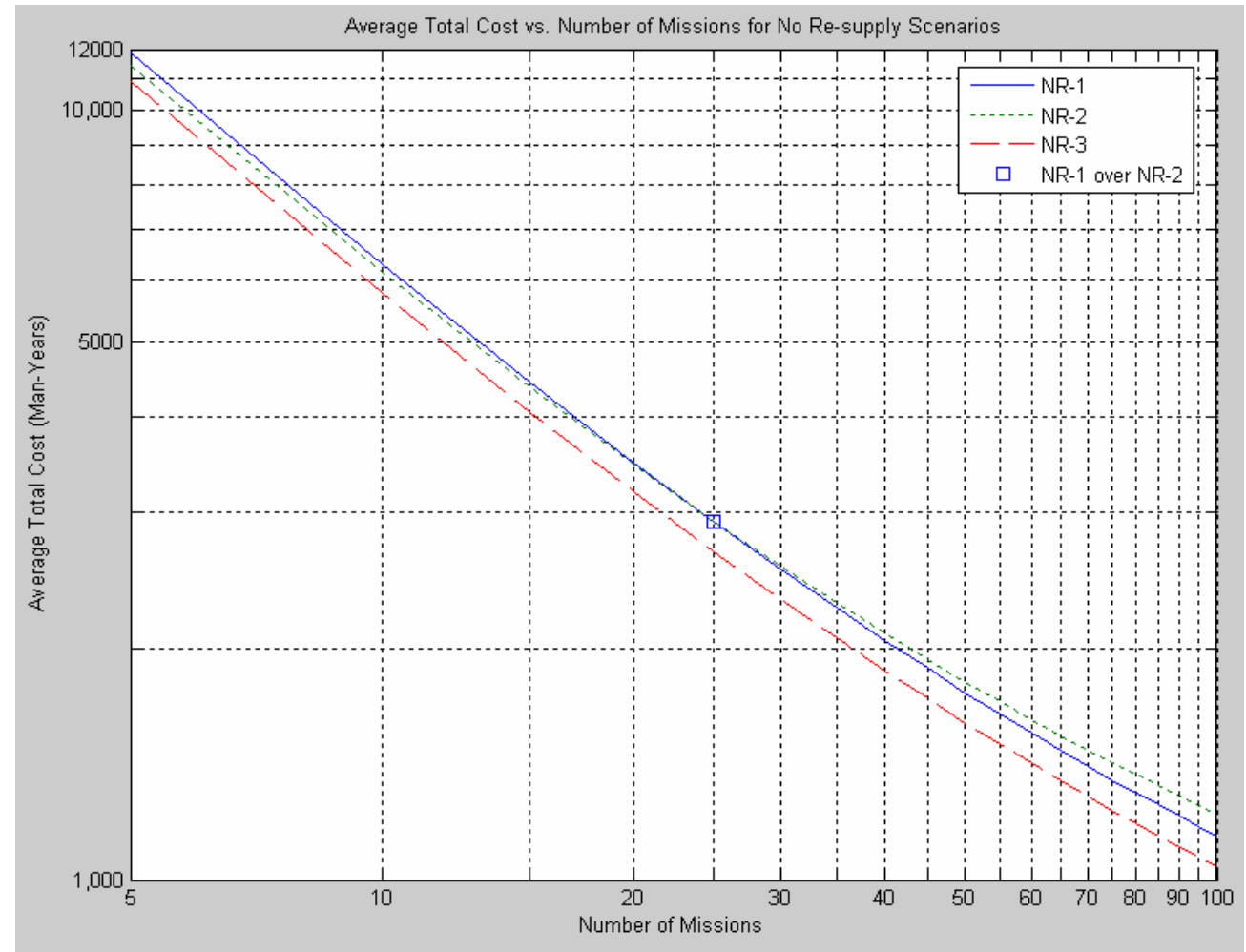

Figure 14: Average total cost vs. number of missions for no re-supply scenarios.

The average total cost for NR-3 is consistently lower because the inert mass of the PM-E is less than the counterpart in NR-1, and the number of PM-Es required in NR-3 is half that of NR-2. Focusing on NR-1 and NR-2, there is a crossover point at which NR-1 begins to have a lower average total cost per mission than NR-2. This point occurs at about 25 missions, as marked in figure 14 by the square. For 5 
missions, NR-2 has a smaller average total coast than NR-1 by about $4 \%$ per mission; at 100 missions, NR-2 grows to be more expensive than NR-1 by about $7 \%$ per mission. In 2005 dollars, NR-2 changes from being $\$ 525$ million less than NR-1 to about $\$ 1.8$ billion dollars more. The crossover occurs because the amortized nonrecurring costs for NR-1 are sufficiently reduced, and the recurring costs for NR-2 begin to compound as a result of the extra PM-E. For the NR-1 and NR-2 scenarios, the difference is sufficiently small compared to potential errors within the cost and vehicle sizing models for up to 100 missions.

\subsubsection{Propellant Transfer versus Tank Replacement}

Figure 15 shows the average total cost versus number of missions for the resupply scenarios. The plot shows that propellant transfer and tank replacement are nearly equal, but for less than 10 missions propellant transfer is slightly lower. The square on the plot marks the crossover point at 10 missions, where tank replacement becomes more economical.

For 5 missions, the tank replacement scenario is only $0.3 \%$ more expensive per mission than propellant transfer; this small difference is only 40 Man-Years per mission, or in 2005 dollars $\$ 9.8$ million per mission. Over all 5 missions, the total difference is less than $\$ 50$ million. For more than 10 missions, tank replacement is lower. However, the average total cost of propellant transfer is higher than tank replacements by no more than $3 \%$ for less than 50 missions. In 2005 dollars, that translates to less than $\$ 600$ million total. At 250 missions, this difference grows to 
$6.5 \%$ or $\$ 2.3$ billion, but 250 missions are well beyond a reasonable number of missions to attempt.

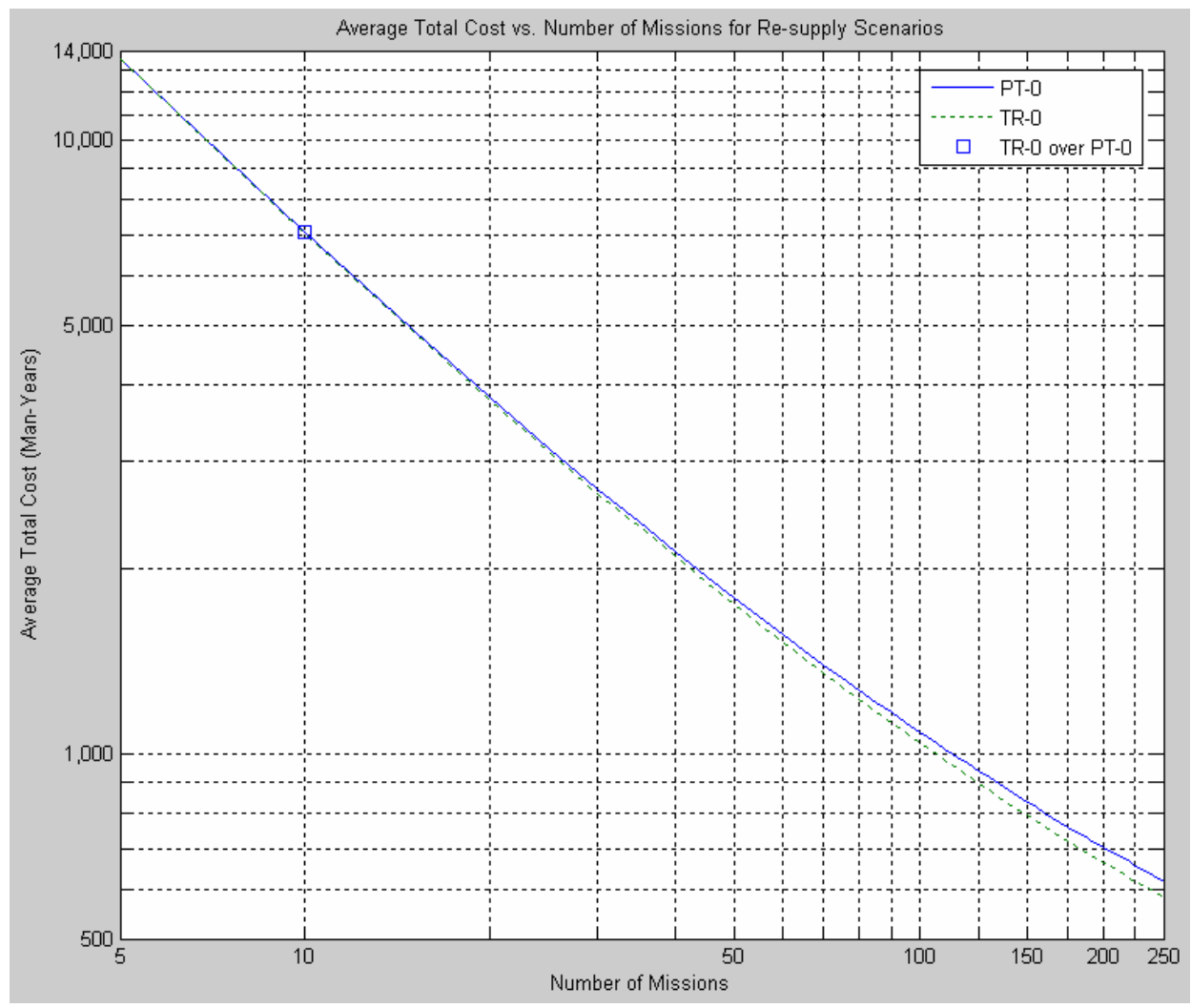

Figure 15: Average total cost vs. number of missions for re-supply scenarios.

For the analysis shown in figure 15, the propellant transfer loss and additional tank replacement hardware were $0 \%$ to ignore inefficiencies for a clear comparison of the ideal cases. For the impact of the respective inefficiencies on the two methods, section 4.4.1 examines the effect of increasing propellant transfer loss, and section 4.4.2 investigates the result of introducing additional tank replacement hardware into the scenario.

The difference between propellant transfer and tank replacement is negligible. The decision for a preferred choice will be based on other considerations. For example, one method may provide better contingency operations and risk reduction. 
Also, the up-front development costs would be a significant driver to influence which direction is affordable in the short-term.

\subsubsection{No Re-supply Versus Re-supply}

The plot of average total cost versus number of missions is repeated in figure 16a to compare no re-supply to the two re-supply options. Of the no re-supply scenarios, only NR-3 is used because it is the most economical and feasible of the no re-supply architectures, and only the ideal cases for the re-supply options are used.

The key information within the plot is the location of the two crossover points, shown in detail in figure $16 \mathrm{~b}$. The first crossover point, shown as a square, highlights where tank replacement becomes more economical than NR-3 at 105 missions; whereas, the second crossover point, shown as a triangle, is at 145 missions, when propellant transfer becomes better than NR-3. Propellant transfer requires $38 \%$ more missions than tank replacement to gain an advantage over NR-3. The sensitivity of the crossover points is explored in detail in section 4.4 for changes to several variables. 


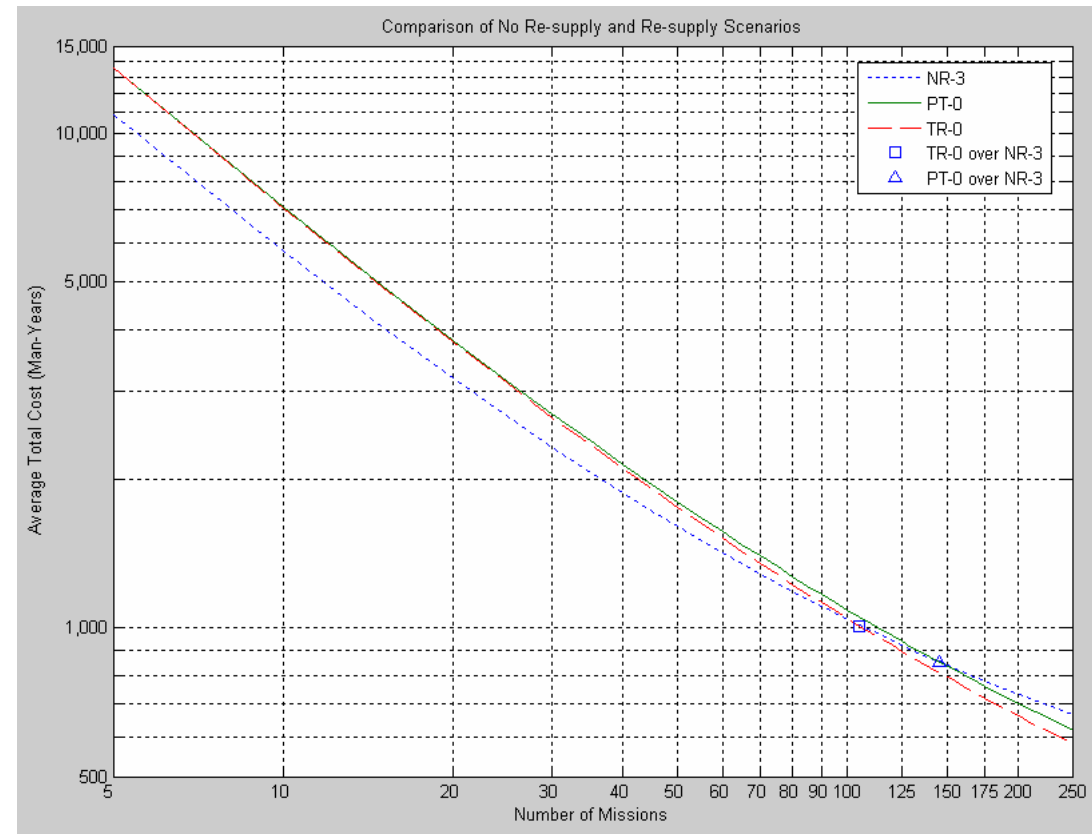

Figure 16a: Comparison of average total cost for no re-supply and re-supply.

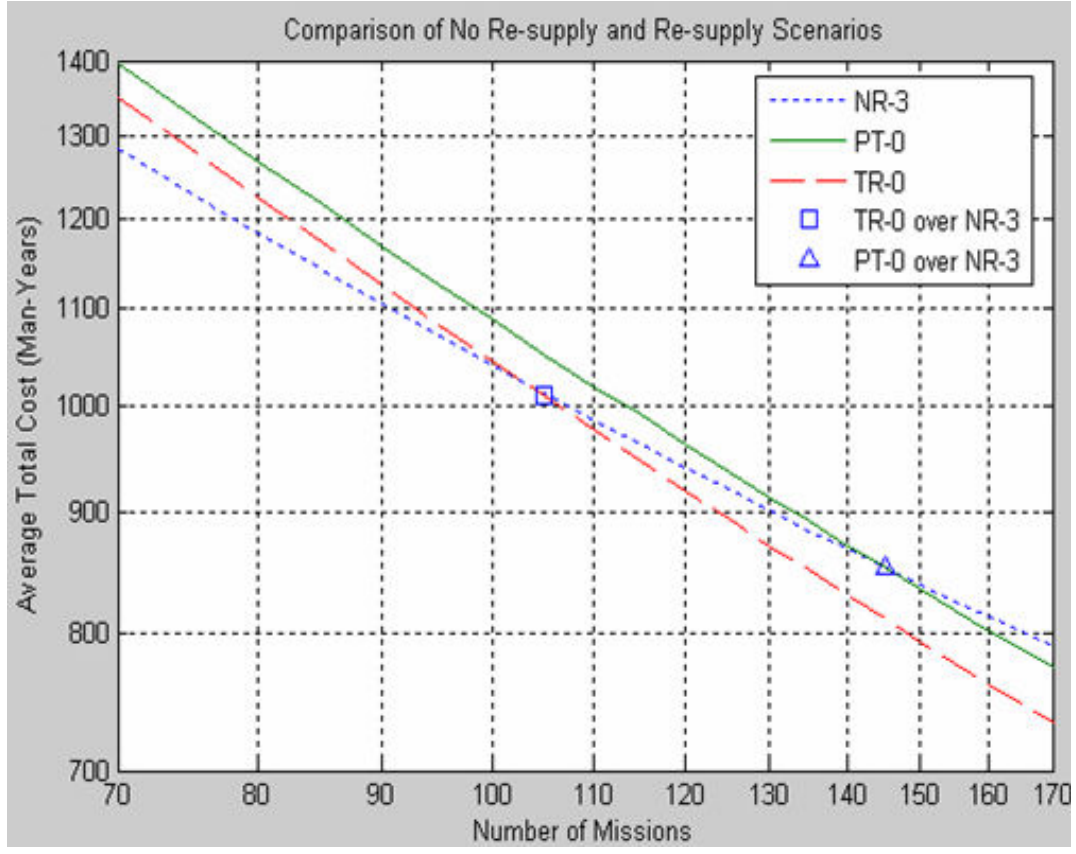

Figure 16b: Detailed comparison of average total cost for no re-supply and re-supply.

At 5 missions, TR-0 is about $25 \%$ more costly than NR-3; this is about 2700 Man-Years per mission, which is $\$ 645$ million per mission and $\$ 3.2$ billion total. At 105 missions, the TR-0 becomes a quarter of a percent better than NR-3, which yields 
a total savings of $\$ 60$ million. This negligible savings continues to grow and exceeds $5 \%$ or $\$ 1.6$ billion at 150 missions and $15 \%$ or $\$ 5.1$ billion at 250 missions.

Similarly, PT- 0 begins as $24 \%$ or $\$ 3.1$ billion more expensive than NR-3. The average total cost of PT- 0 decreases with more missions at a slower rate than TR-0. At 145 missions, PT- 0 is $0.1 \%$ or $\$ 37$ million less than NR-3. At 250 missions, the difference swells to $7.6 \%$ or $\$ 2.8$ billion.

\subsubsection{Comparison Summary}

The preferred no re-supply scenario is NR-3, which is with a single expendable in-space propulsion module (PM-E) and a direct Earth reentry. The selection of NR-3 is independent of the number of missions to be attempted because this scenario is always more cost effective than the NR-1 and NR-2 scenarios.

The preferred re-supply method is difficult to ascertain because of the virtually equal costs for less than 50 missions. If the number of possible missions is expanded to more than 100 missions, then tank replacement is more affordable.

When comparing no re-supply and re-supply, the number of missions that are to be attempted heavily influences the decision. In all likelihood, the future lunar exploration program will be used as a precursor and learning instrument for missions to Mars and beyond, potentially limiting the number of attempted missions to much less than 50 . This strongly supports no re-supply as the preferred option, specifically NR-3. However, if long-term exploration of the moon is adopted and the number of attempted missions is increased to a number similar to the Shuttle Transportation System (STS), then 100 missions could drift into the realm of achievability. At 105 
missions, no re-supply and tank replacement are nearly equal with a slight edge towards tank replacement.

The desire to expand exploration beyond the moon introduces additional factors for consideration. Regardless of the number of missions, the selection of a resupply method for lunar missions would leverage the technology and experience for Martian missions and introduce commercial sector alternatives to reduce development and production expenses, which are discussed in section 5.3. The specific re-supply method will further depend on sensitivity to certain variables, examined in section 4.4 .

\subsection{Sensitivity Analyses}

For all of the sensitivity analyses, a crossover point from one scenario being more cost effective than another is used to provide a convenient basis for comparison. As is the case for several of the studies, the crossover point can occur well beyond 50 or 100 missions, putting the number of missions for a crossover outside of the range of a reasonable number of missions that will actually be attempted. This occurrence is overlooked for the purpose of a direct and consistent assessment. In reality, the actual number of missions that are attempted, regardless of the scenario, will likely be much less than 50 or 100 . However, as explained in section 4.3.4, other mitigating circumstances could influence the acceptable number of missions or preferred architecture. 


\subsubsection{Propellant Transfer Loss Variation}

The highlighted propellant transfer losses were selected to cover a large span. The $0 \%$ loss was considered a baseline to demonstrate the ideal case. The $2 \%$ and $4 \%$ cases were included to demonstrate the cost-to-benefit relationship of a small change in the amount of transfer loss. Additional transfer losses were evaluated to provide continuity and further understanding of the effects.

The number of reusable elements is independent of the propellant transfer loss; the sensitivity to the number of missions for which a reusable element can be reused is considered in section 4.4.4. However, the PM-E and PTM depend on the amount of transfer loss. This is due to the number of required PTMs increasing with transfer loss because the amount of propellant actually delivered by the PTM decreases, and the number of PM-Es increases because one PM-E is required for every two PTMs that need to be delivered to LLO.

The key parameter to measure the sensitivity to the percentage of propellant transfer loss is the change in the crossover point. The crossover for this sensitivity study is defined as the number of missions necessary for propellant transfer to become more cost effective than NR-3. To capture as many of the crossover points as possible for better comparison, the number of attempted missions was increased to 1000. Figure 17 shows the location of the crossover point for all of the propellant transfer losses that were considered. 


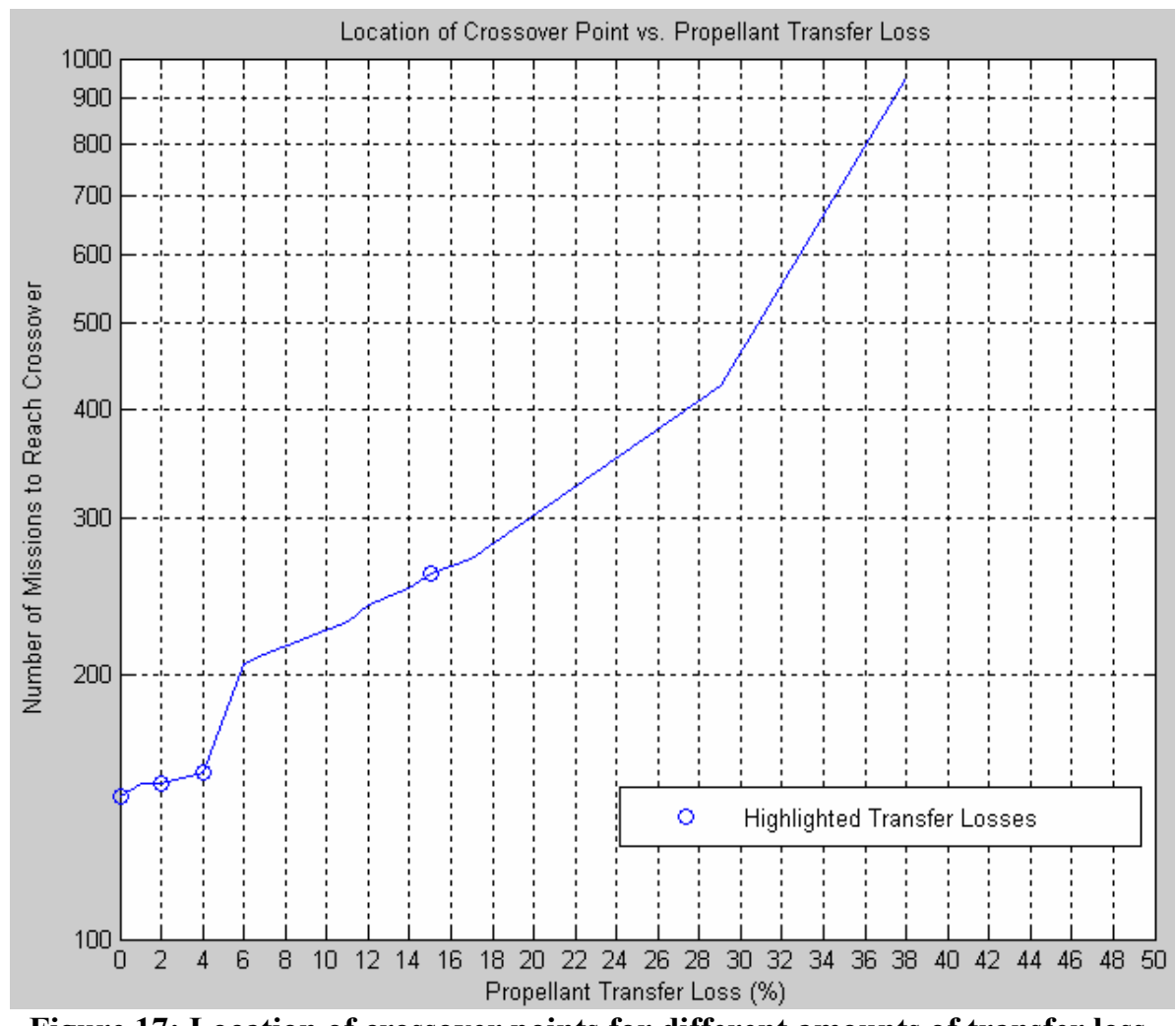

Figure 17: Location of crossover points for different amounts of transfer loss.

The crossover points for $44 \%$ and $50 \%$ transfer loss exceed 1000 missions. The crossover points show several regions of linearity. The entire plot is not a continuous region of linearity because as the transfer loss increases the number of elements that need to be produced can increase rapidly or slowly depending on the amount of each PTM that is being consumed; this is explained in detail at the end of section 4.2.2.

Anticipated propellant transfer losses that have been suggested are about $0.5 \%$ for oxygen and 3.3\% for hydrogen ${ }^{9}$. For the PM-R, the total transfer loss would be $252 \mathrm{~kg}: 120 \mathrm{~kg}$ of oxygen and $132 \mathrm{~kg}$ of hydrogen; this is about $0.5 \%$ of the total PM$\mathrm{R}$ usable propellant. The transfer loss to the lander would be $47 \mathrm{~kg}$ of oxygen and 70 $\mathrm{kg}$ of hydrogen or about $1 \%$ of the total usable propellant. The low percentage of the total propellant load is a result of the high oxidizer to fuel ratio on each vehicle. The 
amount of transfer loss suggested in ref. 9 was for a system receiving about 20 tons of propellant, which is similar to the PM-R in this study. The liquid oxygen (LOX) and liquid hydrogen (LH2) percents are different because of different physical properties, especially the boiling point and density.

\subsubsection{Variation of Percentage of Additional Hardware for Tank Replacement}

As explained in section 4.1.3, the additional hardware is calculated as percentage of the tank replacement modules (TRMs) that are integrated with the vehicle: 2 TRMs for the PM-R and 1 TRM for the lander. Similar to the propellant transfer losses, the low, medium, and high percentages of additional tank replacement hardware were highlighted. Also, the additional low value should provide insight into the benefit of a small decrease in the required additional hardware for tank replacements. Other additional hardware percentages are evaluated to further understand the behavior.

The required number of all elements is the same as the baseline of $0 \%$ additional hardware for all amounts of additional hardware, except for $20 \%$ or more. The increase at $20 \%$ additional hardware is caused by the decrease from two TRMs to one that the PM-E can deliver to LLO, as explained in section 4.2.1. This doubles the number of PM-Es required for each attempted mission. The number of TRMs is independent of the additional hardware because the TRMs were sized in all tank replacement scenarios such that two TRMs are necessary for one PM-R and one TRM for the lander. 
Figure 18 shows how the crossover point changes for different amounts of additional hardware. The crossover point is defined as the number of missions at which a particular tank replacement scenario becomes more economical than NR-3. The crossover points for $20 \%$ and above exceed 1000 missions.

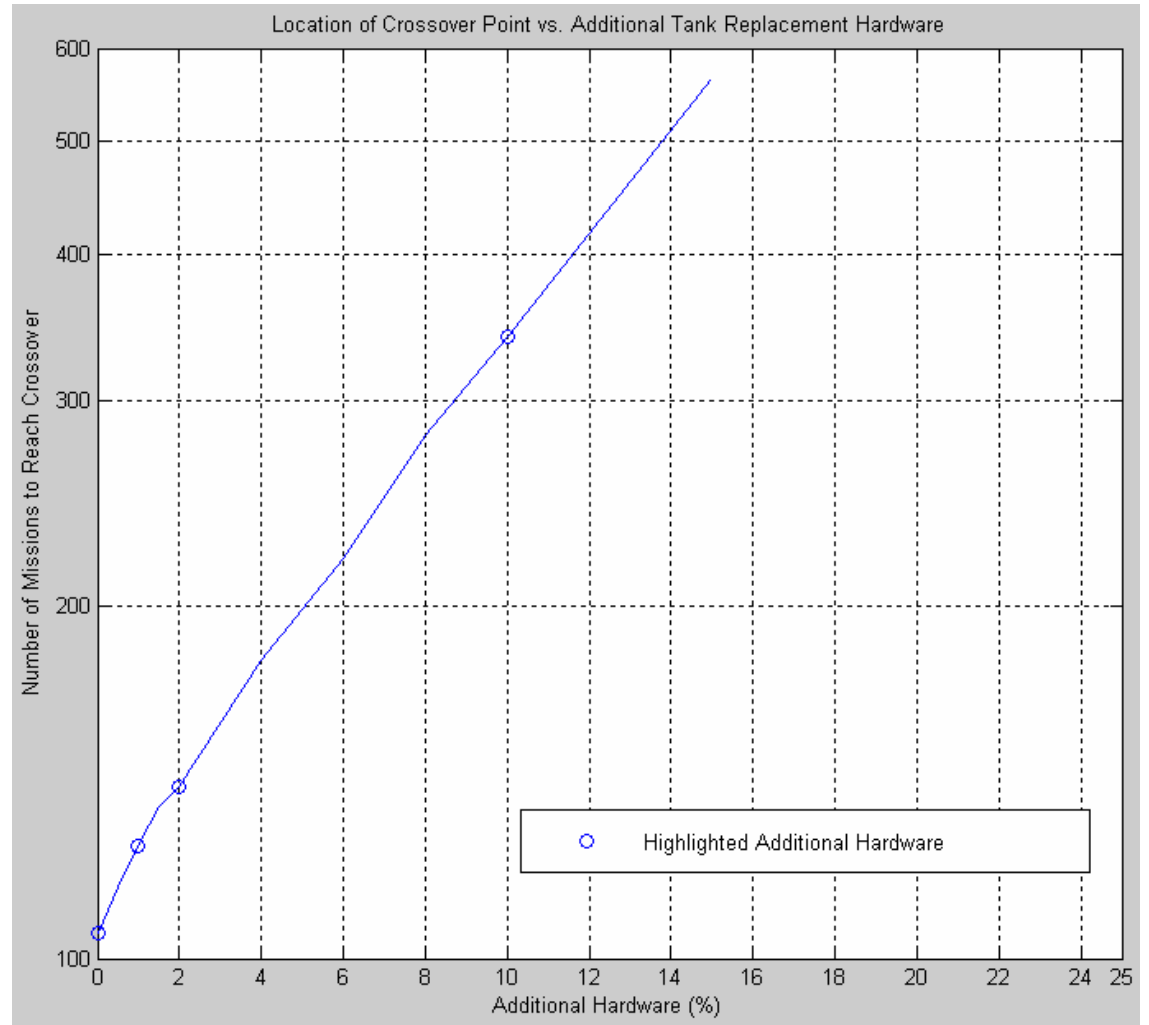

Figure 18: Location of crossover points for different amounts of added hardware.

Since the tank replacement scenarios fixed the number of TRMs to re-supply the PM-R and lander, the only result of increases in the additional hardware is increased element masses, as explained in section 4.1.3. The crossover point increases with increases in additional hardware because the elements for larger amounts of additional hardware are more expensive to develop and produce. For this reason, the shape of the curve in figure 18 is very similar to the shape in figure 13. 
Within the current literature on propellant re-supply, there are no estimates for additional tank replacement hardware. To approximate a percentage, the CAD model shown in figure 9 of the structural supports, not including the TRMs, was used. The total mass of the support structure is estimated between $175 \mathrm{~kg}$ for all graphite composite to $300 \mathrm{~kg}$ for an all aluminum-lithium alloy. This results in a range from about $0.5 \%$ to $1.25 \%$ additional tank replacement hardware.

\subsubsection{Inert Mass Variation}

The inert masses of each element in all scenarios were varied separately by $+/-$ $25 \%$ to determine which elements are most sensitive; the inert mass is the gross vehicle mass without payload minus the usable propellant mass. The inert mass was selected for a sensitivity study because it is a primary factor in the calculation of development and production costs. For consistency, the sensitivity was determined by how much the crossover point shifts as a result of the changes. Combined effects of changing all inert masses within a scenario at once were not investigated since the objective was to determine which element is most sensitive. However, the obvious conclusion is that increases or decreases to all inert masses will simply result in more substantial overall effects.

For the no re-supply scenarios, all three scenarios were compared to the PT-0 scenario as a base of reference. The crossover point is defined as the number of missions required for PT-0 to have a lower average total cost than the respective no re-supply scenario; a larger crossover implies a more cost effective no re-supply 
scenario. The bar graph in figure 19 shows how the crossover point changes for each element compared to the baseline from section 4.3.3.

The five columns for each scenario are the baseline, $+25 \%$ inert mass of the PM-E, $+25 \%$ inert mass of the lander, $-25 \%$ inert mass of the PM-E, and $-25 \%$ inert mass of the lander, respectively. As expected, an increase in the inert mass of either element causes the crossover point to decrease; whereas, a decrease in inert mass results in a higher crossover point. From this data, the lander is clearly a more sensitive element in the architecture. Changes to the lander result in about two times the variation from the baseline as compared to the PM-E. The lander is much more sensitive because it represents about $45-60 \%$ of the production costs and $78-84 \%$ of the development costs for the no re-supply scenarios.

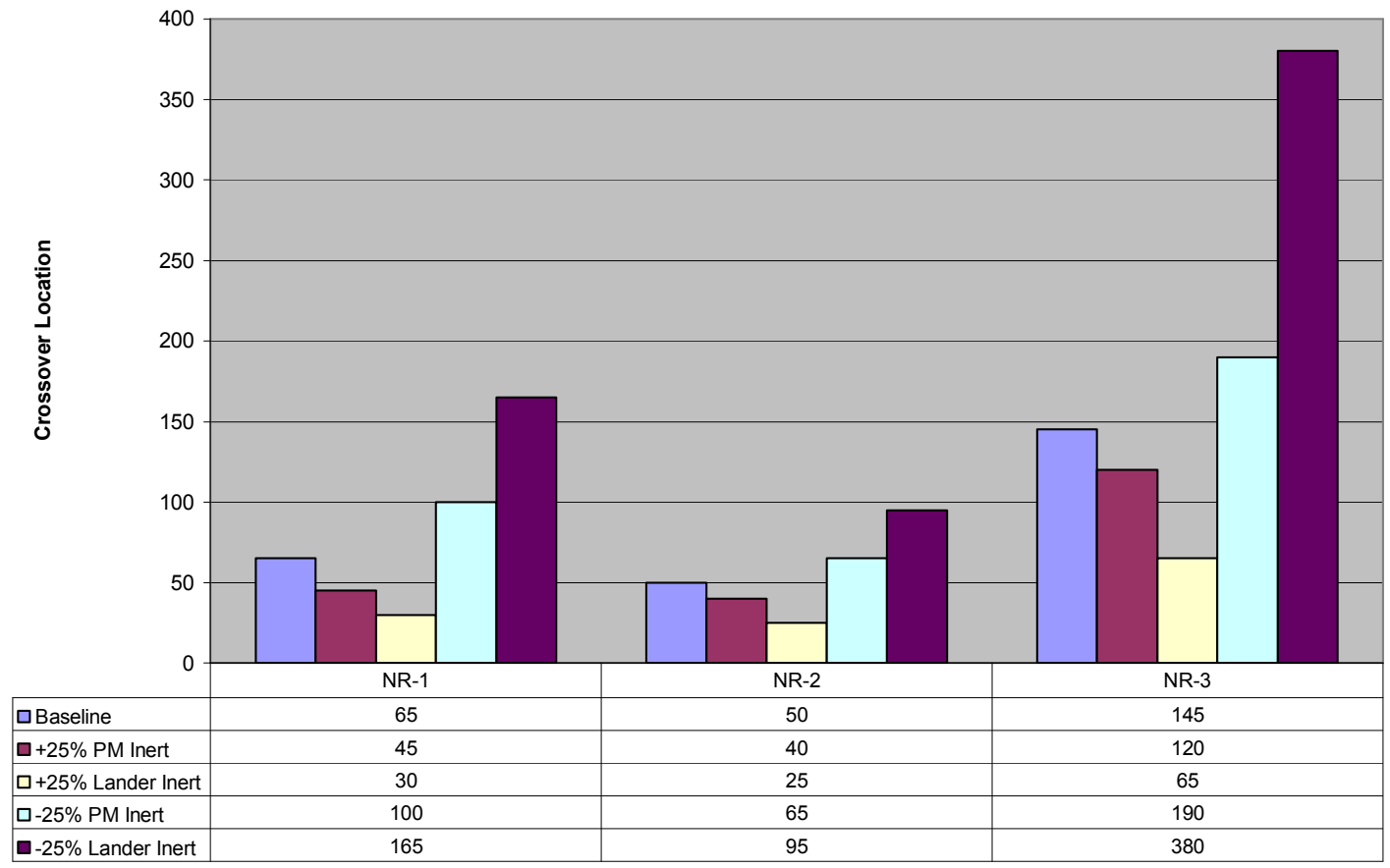

Figure 19: No re-supply scenario sensitivity to inert mass.

Similarly, the effect of changing the inert masses of each architecture element for propellant transfer and tank replacement are shown in figures 20 and 21, 
respectively. All inefficiencies were considered to further demonstrate the effects of large penalties on either method. The crossover for the re-supply options were defined as the number of missions required to reduce the average cost per mission of a re-supply scenario below that of NR-3. The NR-3 scenario was selected because it represents the best of the no re-supply options, as explained in section 4.3.1. Any crossover not displayed on the plots occurs beyond 1000 missions.

For both re-supply methods, changing the inert mass of a single element results in a shift of the entire line shown in figures 17 and 18 and reproduced on the following figures as the baseline. Changes to the re-supply modules, PTM and TRM, respectively, do not cause a shift from the baseline. This is a result of the re-supply module comprising the smallest portion of the production and development costs. As seen with the no re-supply elements, the lander inert mass sensitivity is the greatest. For propellant transfer scenario, an inert mass change in the lander of $25 \%$ results in about a 35\% shift in the crossover point; the shift is down for a decrease in inert mass, and vice versa. For the PM-E, the effect is not as great as for the lander; it is only 22\%. The PM-R has an even smaller jump of about $9 \%$. The tank replacement results are the same for the PM-R of about 9.5\%. The PM-E and lander effects are less than the propellant transfer counter-parts. For the PM-E, the change in inert mass causes a change to the baseline of about $16 \%$, and the lander change is about $27 \%$.

The lander is the most sensitive element due to the high sensitivity of any lunar architecture to the amount of mass that lands on the lunar surface. The PM-E is the next most sensitive because each mission requires at least one; whereas, the PM-R is less sensitive because one is only needed every fifth mission. The difference 
between the propellant transfer and tank replacement scenarios is based on the costs being related to vehicle inert masses. Since the propellant transfer element inert masses are the same for all transfer losses and the tank replacement elements have a smaller inert mass for most of the inefficiencies, each propellant transfer element is more sensitive than the tank replacement counterpart.

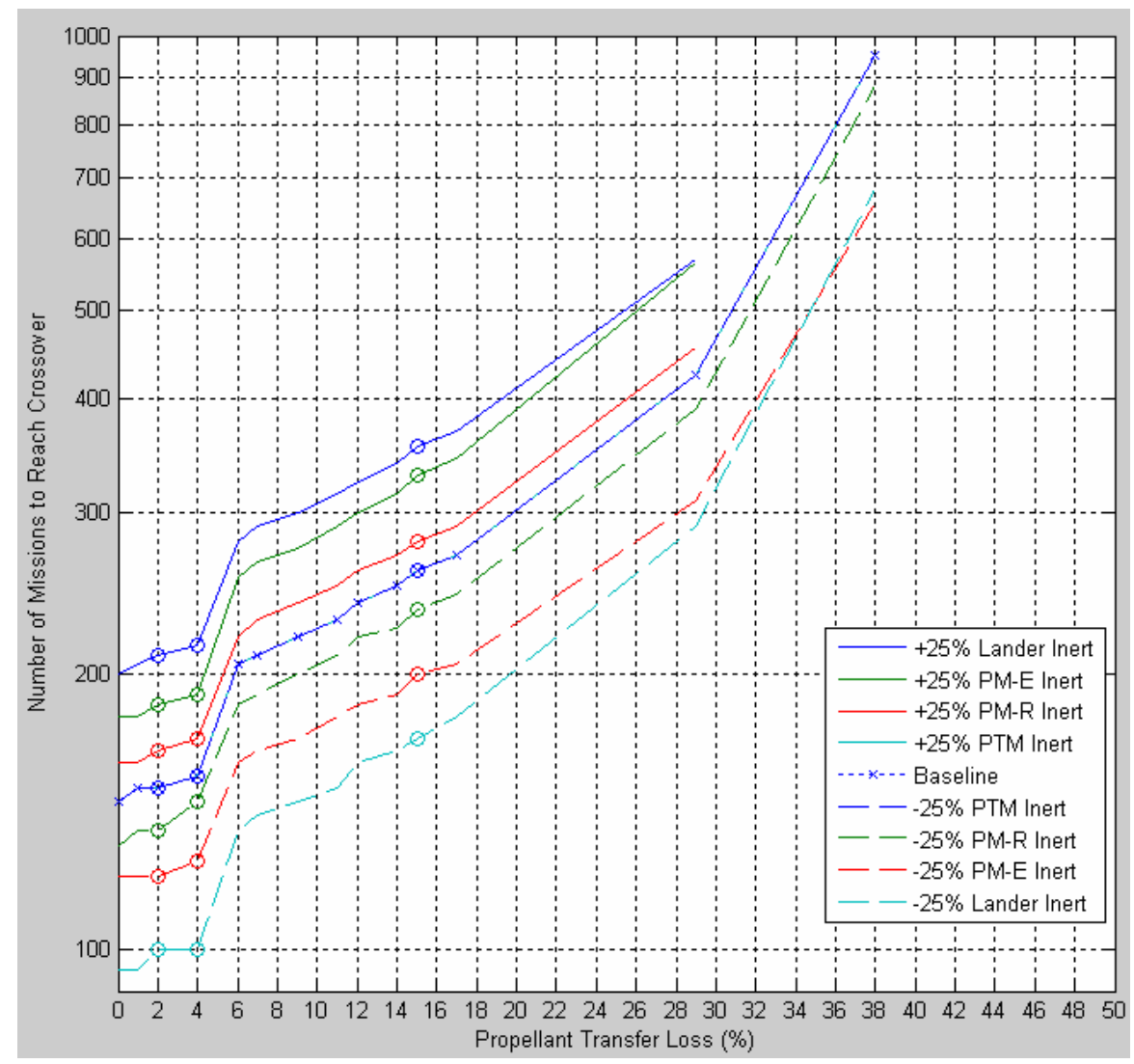

Figure 20: Propellant transfer scenario sensitivity to inert mass. 


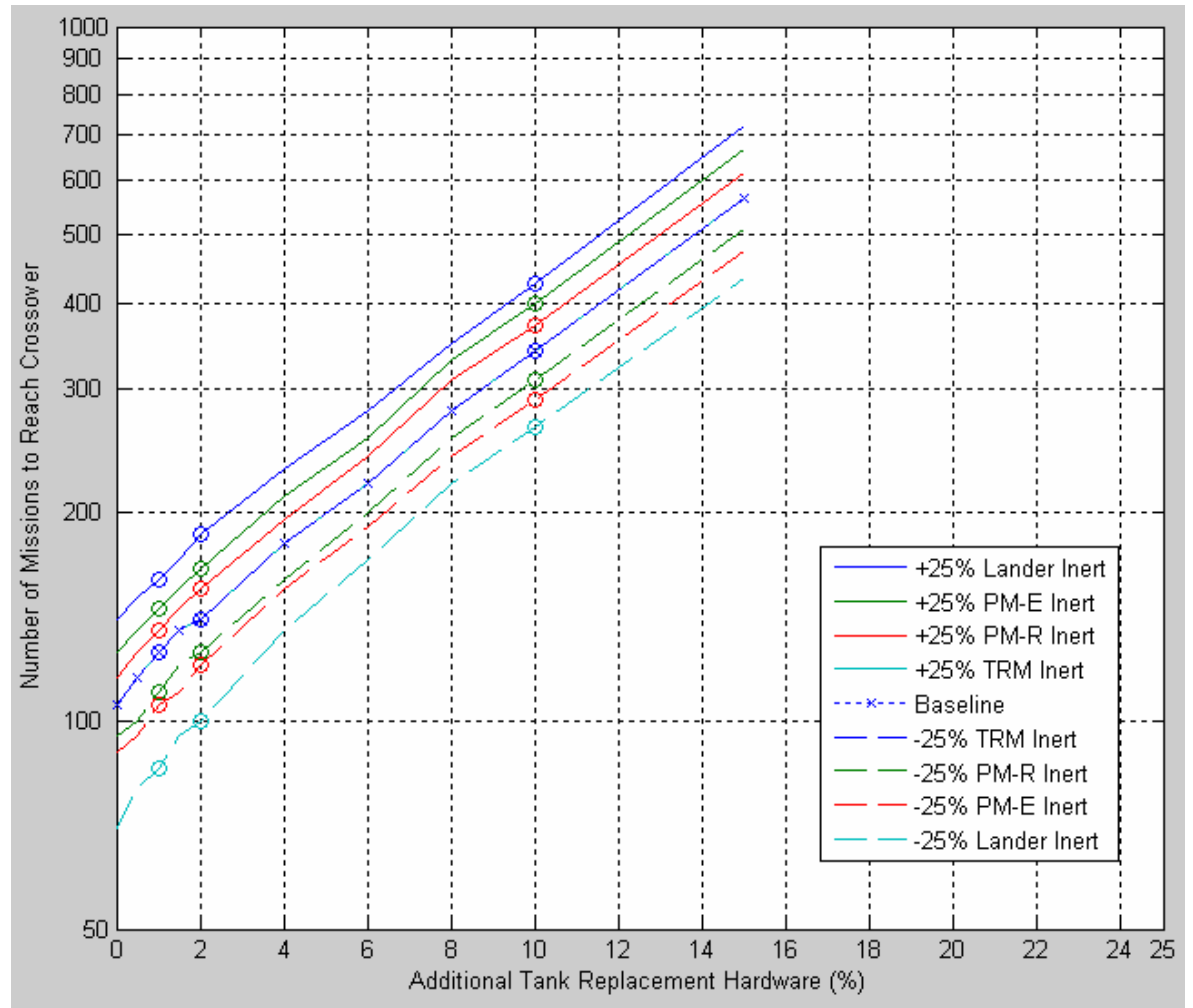

Figure 21: Tank replacement scenario sensitivity to inert mass.

\subsubsection{Reusability Variation}

In figures 22 and 23 , the sensitivity of the re-supply scenarios to the reusability of the PM-R and reusable lander is shown. The reusability was tested at 2, 5,10 , and 15 missions per reusable element; the baseline throughout has been 5 missions. The PM-R and lander were always assumed to be replaced at the same time, during the first mission of a new campaign. The length of a campaign is the number of missions for which the reusable elements can be used.

In all re-supply scenarios for both methods, the number of missions to make re-supply more cost effective than NR-3 significantly reduces by increasing from 2 missions per reusable element to 5 missions. By increasing reusability by 3 missions, the crossover point decreases by nearly fifty percent; however, further increases to 10 
or 15 missions per set of reusable elements only causes a minor incremental decrease in the number of missions for the crossover. Such increases in reusability may result in additional costs not considered here and may erode any added benefit. For example, reusability was changed independent of the development factor. In order to increase the reusability of an element, the difficulty of development could increase.

For propellant transfer, a reusability of only 2 missions requires on average about $95 \%$ more missions to be more cost effective than 5 missions. Increasing from 5 missions to 10 missions causes a $29 \%$ reduction in the crossover point, and 15 missions for reusability results in a further $10 \%$ reduction. For tank replacements, the sensitivity to number of reuses for the PM-R and lander is less sensitive. An increase of $70 \%$ occurs for 2 reuses as compared to 5 reuses. Increasing to 10 reuses lowers the crossover point by $22 \%$, and an increase to 15 reuses causes a $8 \%$ reduction as compared to 10 reuses. The tank replacement scenario is less sensitive because the per element production costs are significantly lower. For the PM-E, the tank replacement version is about $3 \%$ less to produce. The PM-R is about $28 \%$ less, the lander is about $13 \%$ less, and the TRM is about $19 \%$ less than the PTM. The total effect of these differences results in propellant transfer being more sensitive to changes than tank replacement. 


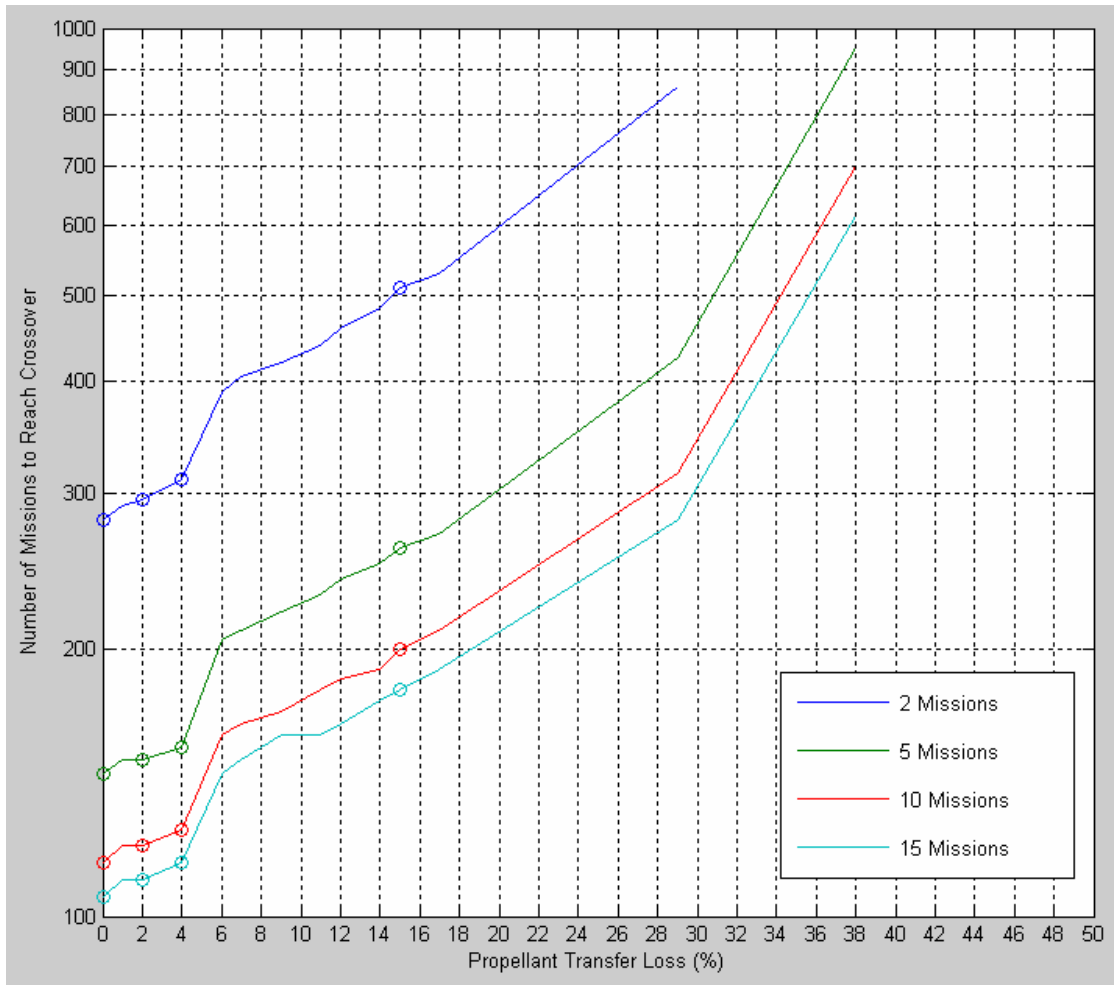

Figure 22: Propellant transfer scenario sensitivity to reusability.

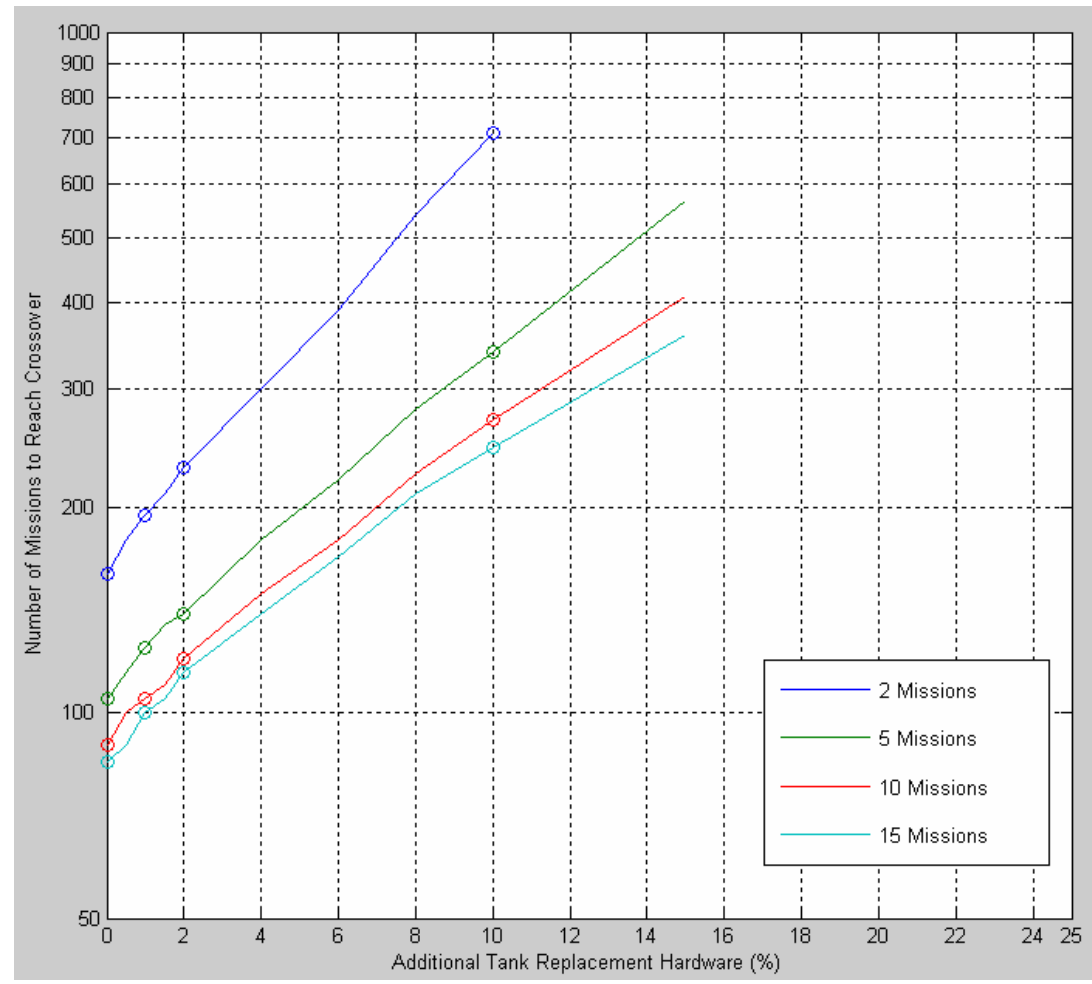

Figure 23: Tank replacement scenario sensitivity to reusability. 


\subsubsection{Production Learning Curve Variation}

The sensitivity of the crossover point to the production cost learning curve parameter was evaluated by changing the factor by $+/-5 \%$. An increase in the learning curve value by $5 \%$ was defined as a worsening in the amount of learning gained by increased production, i.e. the learning factor shifts towards a value of one. The learning curve factor variations were applied to all elements simultaneous based on the assumption that all elements are produced using common manufacturing techniques and knowledge.

The analysis for the no re-supply scenarios is shown in figure 24 . The learning curve parameter is a significant influence on the cost effectiveness of an architecture. For a 5\% decrease in the learning curve parameter, the factor became closer to zero and causes NR-1 and NR-3 to always be more cost effective than the PT-0 scenario, which was used as a base of comparison. For NR-2, the crossover point is very large, exceeding 900 missions. This shows that the learning curve factor can effectively prevent PT-0 from ever being more affordable than no re-supply. For an increase in the learning curve factor, the parameter becomes closer to one and reduces the crossover point by $50 \%$ for NR-1 and NR-2 and by nearly $2 / 3$ for NR-3.

The high sensitivity for the no re-supply scenarios is the result of a higher number of landers that must be produced; the no re-supply scenarios require 5 times the number of landers as PT-0. As explained in section 4.4.3, the lander is the most expensive element to be produced for both no re-supply and re-supply scenarios. Since the baseline had a learning curve factor closer to one for the no re-supply scenarios and the no re-supply scenarios require many more landers, enhancing the 
learning curve factor significantly increases the amount of savings with each additional lander by more than the savings in PT- 0 .

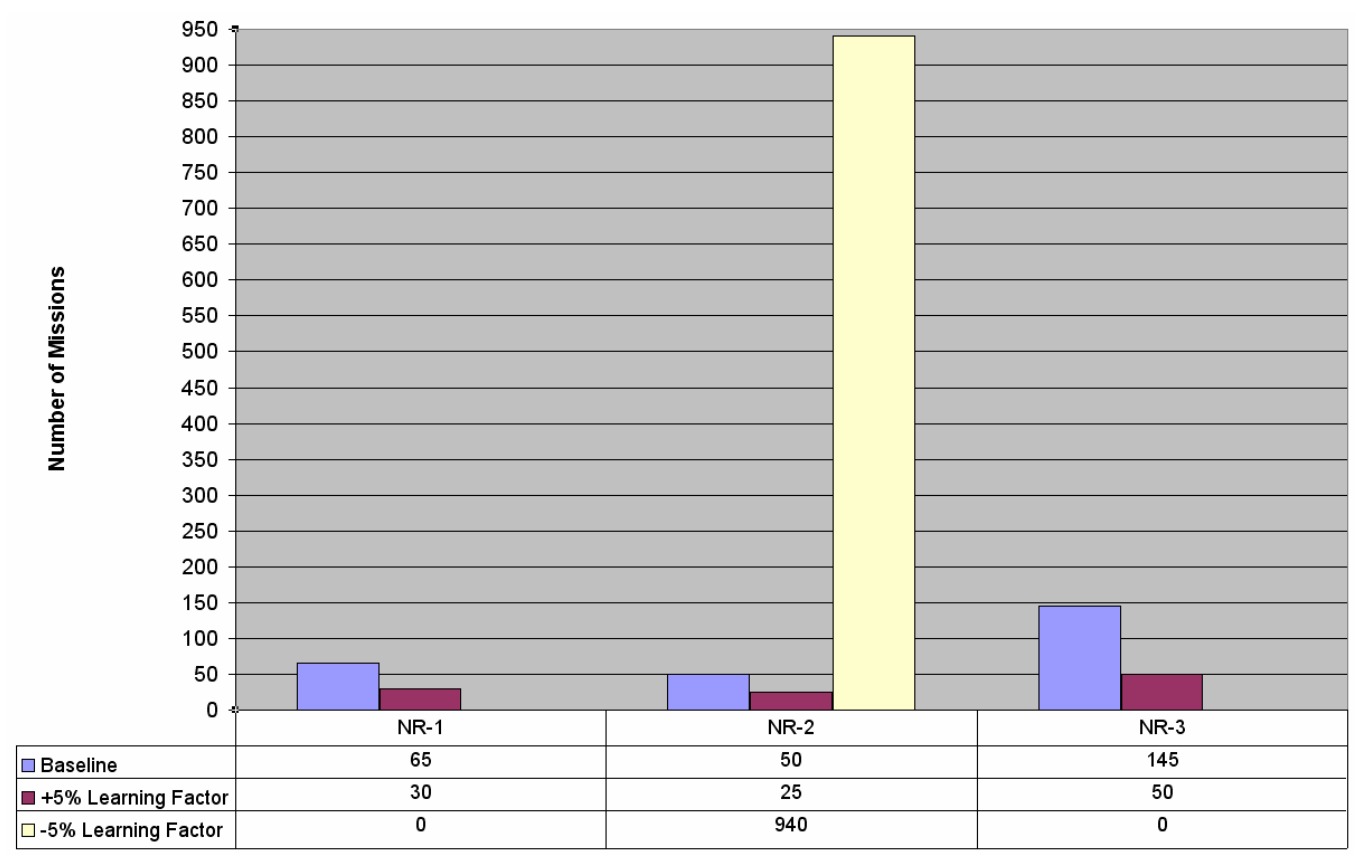

Figure 24: No re-supply scenario sensitivity to learning curve.

Similarly, for the two re-supply methods, figures 25 and 26 illustrate the effects of changing the learning curve parameter for all architecture elements. For + $5 \%$ learning curve, the re-supply scenarios never became better than no re-supply, and the line is not shown because all crossover points exceed 1000 missions. However, enhancing the learning curve reduces the crossover point by as much as two thirds. Also, the 5\% reduction in the value of the learning curve factor allowed the worst cases of inefficiencies to become more cost effective than NR-3; this is the only factor that has created a crossover point for $50 \%$ propellant transfer loss and $25 \%$ additional tank replacement hardware

The basic reason for the re-supply scenarios never out performing NR-3 is that the re-supply scenarios require a very large number of elements that need to be 
produced. Higher production requirements, and therefore production costs, can only be offset by an enhanced learning curve factor. This is why NR-3 is always better than re-supply if the learning curve factor is close to one. As explained in section 3.4, the learning curve factor is linked to the specific vehicle size and total number of production elements. Therefore, it is unlikely that the larger no re-supply elements which have lower totals of production for the same number of missions would have a similar or better learning curve factor.

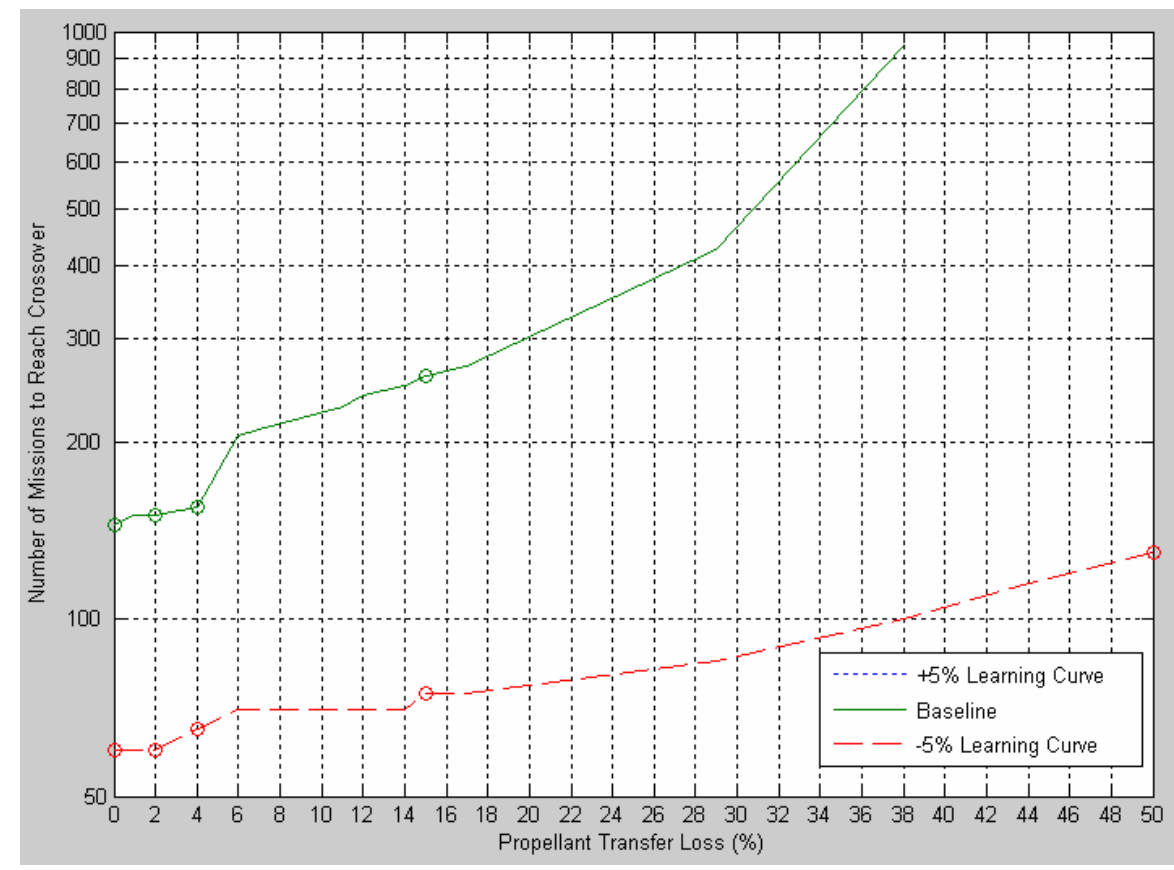

Figure 25: Propellant transfer scenario sensitivity to learning curve. 


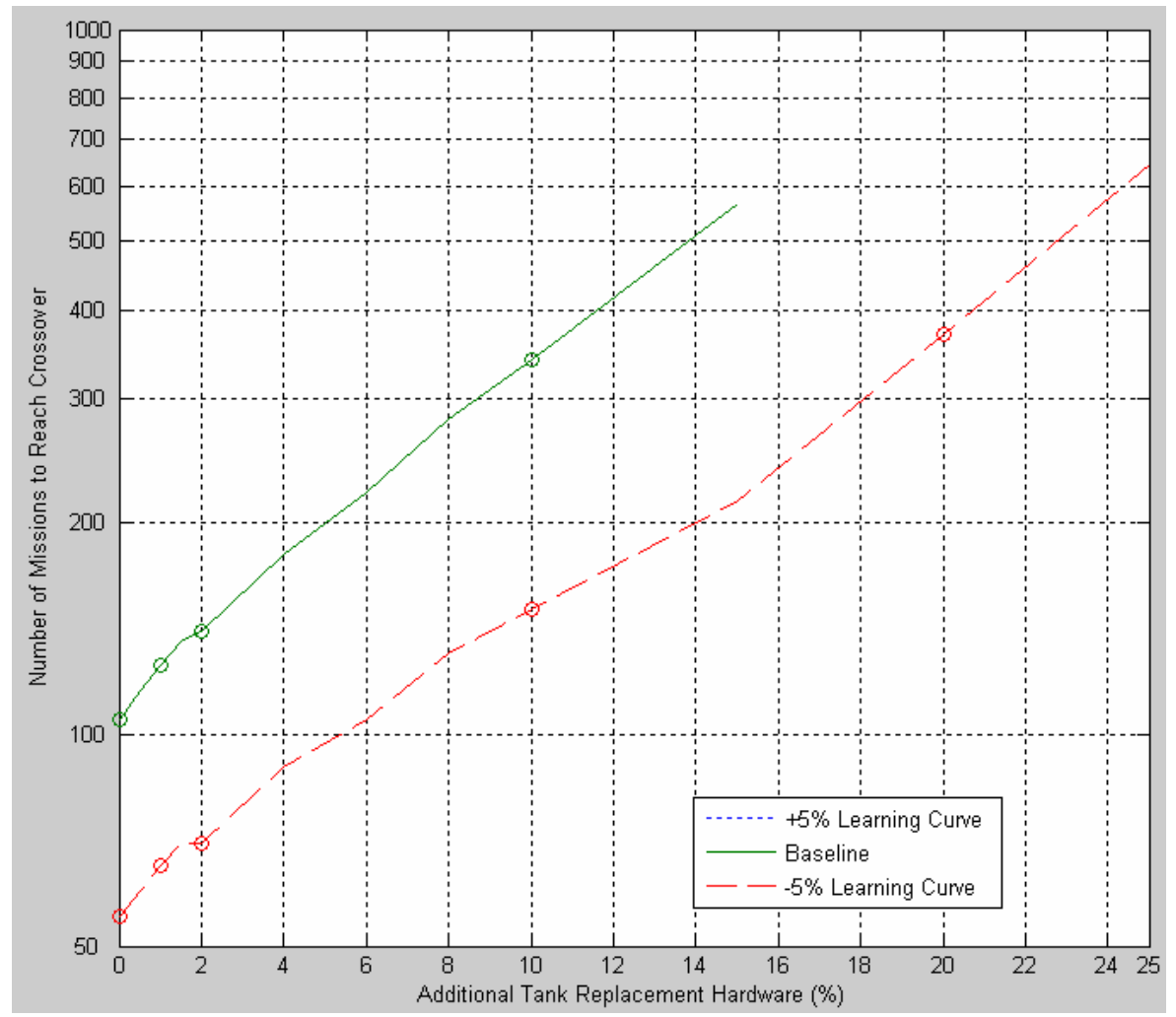

Figure 26: Tank replacement scenario sensitivity to learning curve.

\subsubsection{Team Experience Variation}

The value of the team experience factor was varied for each architecture by $+/-1$, on a scale from zero to ten, for each element. Since several elements might have similar team experience factors because they are design heritage from the same types of projects and vehicles, the analysis was completed by changing the experience factor for all of those "related" elements at once.

For the no re-supply scenarios, only two elements are available to be changed: the PM-E and lander. As shown in figure 27, the effect of changing the experience factor for the PM-E and lander has similar effects to other parameters. The PM-E has a limited sensitivity to the experience factor; whereas, once again, the largest effect is a result of changes to the lander. The lander shows a sensitivity of about 2 times that 
of the PM-E. This is due to the lander being more significant in the development and production costs of each no re-supply scenario.

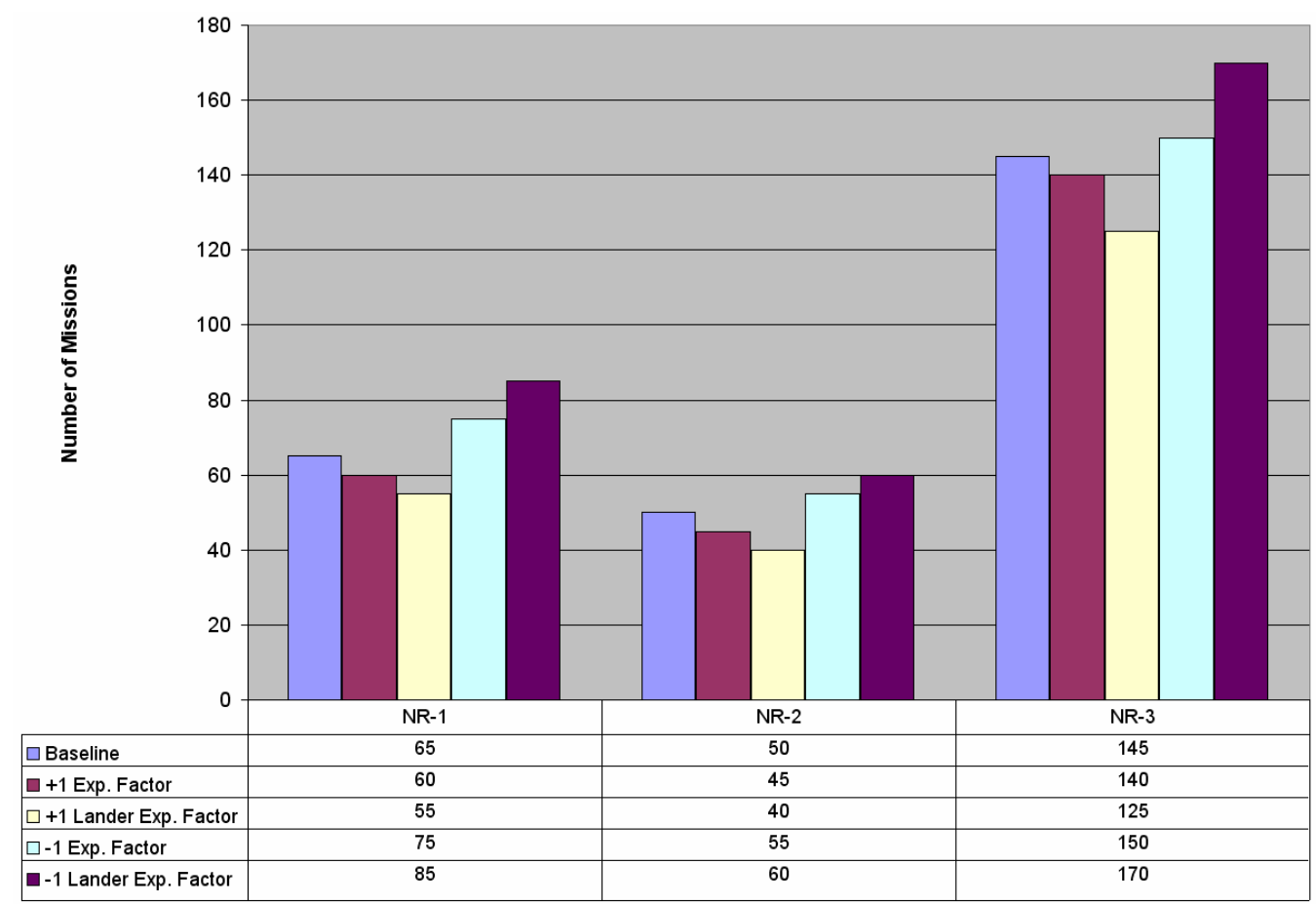

Figure 27: No re-supply scenario sensitivity to experience factor.

For the re-supply scenario, three of the elements are related by common heritage: the PM-E, PM-R, and re-supply module. The lander was changed separately since it has an independent experience-base. Figures 28 and 29 show the result of the sensitivity analyses of the two re-supply methods. For both methods of re-supply, the changes in the experience factor do not cause the worst cases of the respective inefficiencies to become better than NR-3.

For the propellant transfer options, the experience factor of the reusable lander is more significant in shifting the crossover points. The change in the related elements' experience factors causes an average of $+/-7 \%$ shift in the crossover points, and the change to the lander experience factor averaged $a+/ 13 \%$ shift in crossovers. 
For the tank replacement method, the sensitivity of the crossover point is nearly the same for the three related elements as for the lander. The experience factor of the related elements caused an average shift of $+/-8 \%$, and the lander experience factor resulted in $\mathrm{a}+/ 10 \%$ average change in the crossover points. The lander is more sensitive for the propellant transfer method because it has a higher average inert mass than the average inert mass of the tank replacement method.

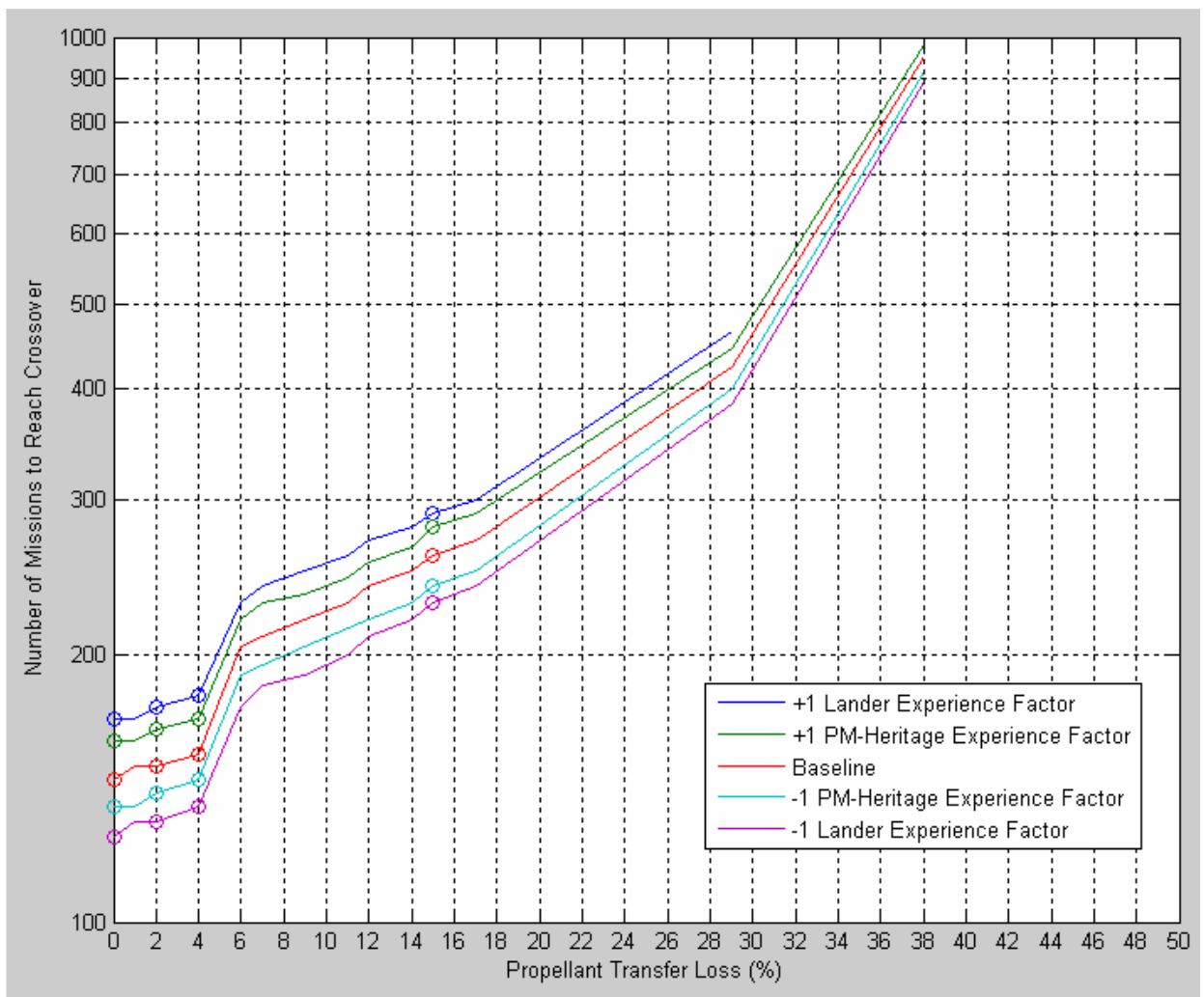

Figure 28: Propellant transfer scenario sensitivity to experience factor. 


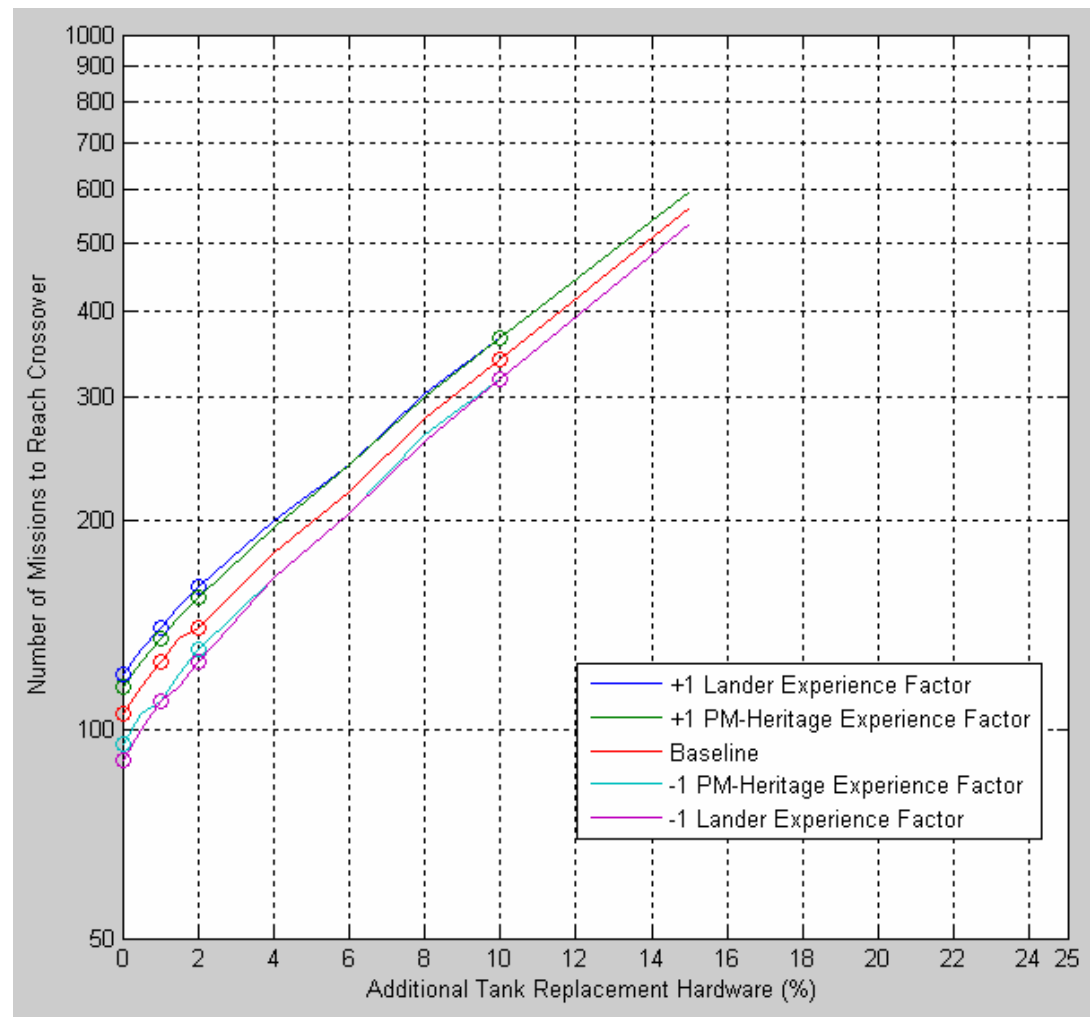

Figure 29: Tank replacement scenario sensitivity to experience factor.

\subsubsection{Development Standard Variation}

The development factor for each of the architectures was varied by $+/-1$. Despite the assumed related experience that was drawn upon for the creation of similar elements, the development factor was varied separately for all elements within an architecture. This is primarily due to the potential difference in technologies and subsystems that are applied to each element.

The results of the no re-supply development factor sensitivity are shown in figure 30. As seen in other analyses, the lander exhibits the most sensitive variation to the parameter that is being altered. An increase to the lander development factor decreases the crossover point by $27 \%$, and a decrease in the factor results in a $31 \%$ increase in the crossover point. This is significantly greater than the PM-E 
development factor, which causes a $-7 \%$ or $+11 \%$ change for increases or decreases to the development factor, respectively. The most realistic values for the development factor are those listed in table 5 as the baseline.

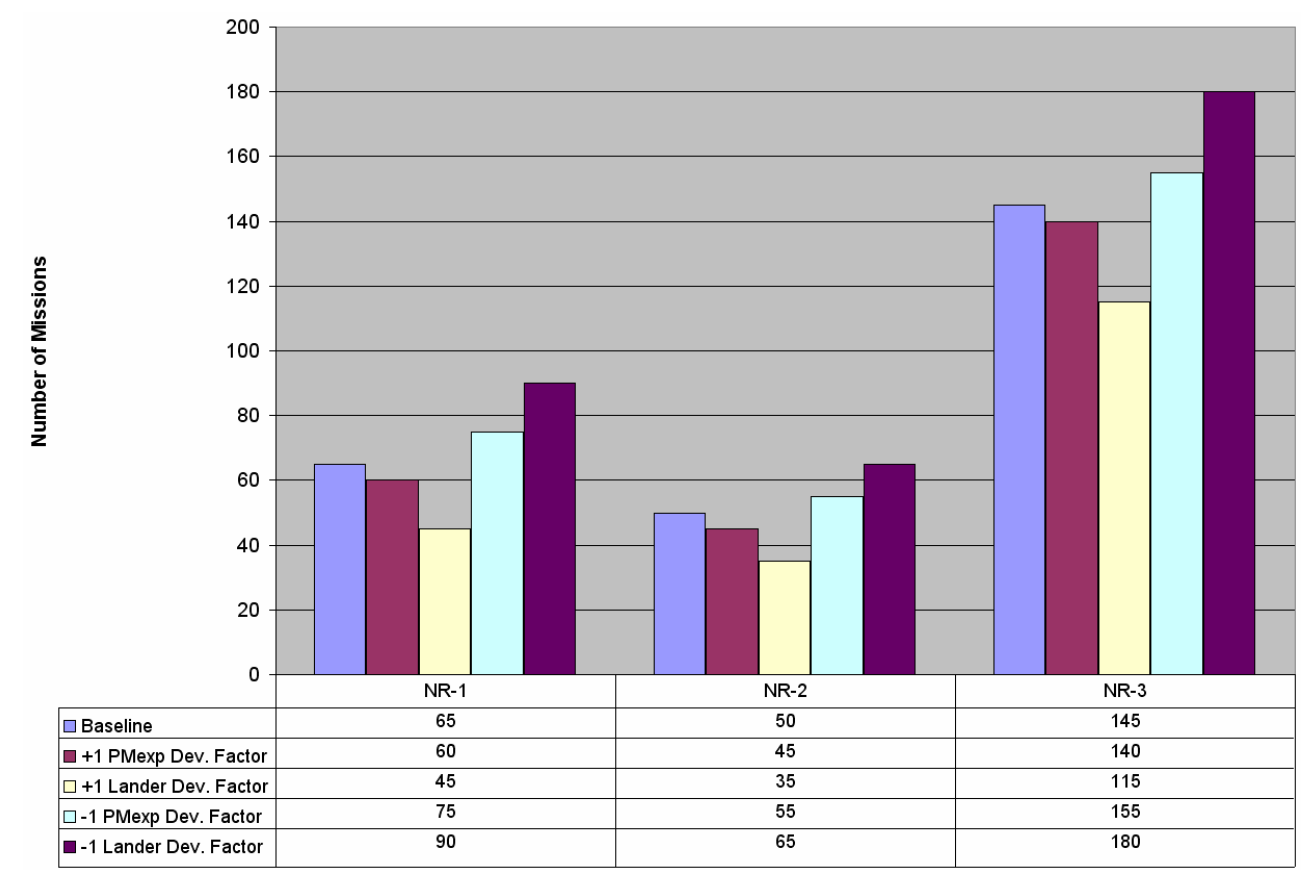

Figure 30: No re-supply scenario sensitivity to development factor.

Similar results for the re-supply scenarios are shown in figures 31 and 32 . Of particular importance, the re-supply modules, PTM for propellant transfer and TRM for tank replacements, result in changes of less than $1 \%$ for changes to the development factor because they represent such a small proportion of the development and production efforts.

The PM-E and PM-R have close effects for the changes in the development factor. For the propellant transfer, the PM-E causes a change of about $+/-4 \%$, and the PM-R results in about a $+/-5 \%$ change. The lander has the greatest impact at $+/-$ 19\%. The tank replacement method has similar results: the PM-E development factor causes $+/-3.5 \%$, the PM-R has $+/-7 \%$, and the lander is $+/-15 \%$. This pattern is 
similar to the sensitivity analysis of other factors. The primary causes are the elements' relative impact on the development and production costs, and the baseline values of the development factor. Other than the lander, the tank replacement elements are more sensitive than the counterparts for propellant transfer because two of the three elements for the replacement tanks have a higher development factor baseline; therefore, any variation would start out higher.

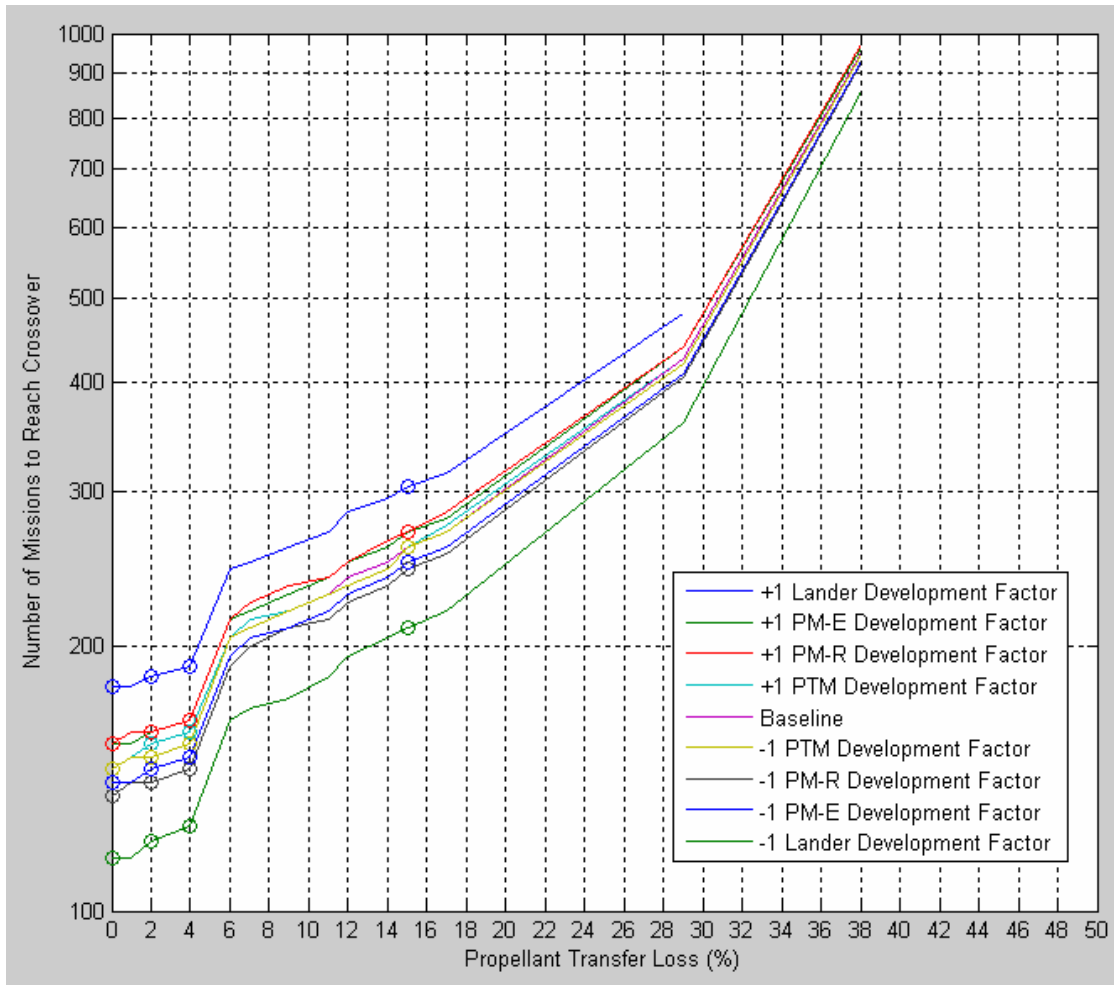

Figure 31: Propellant transfer scenario sensitivity to development factor. 


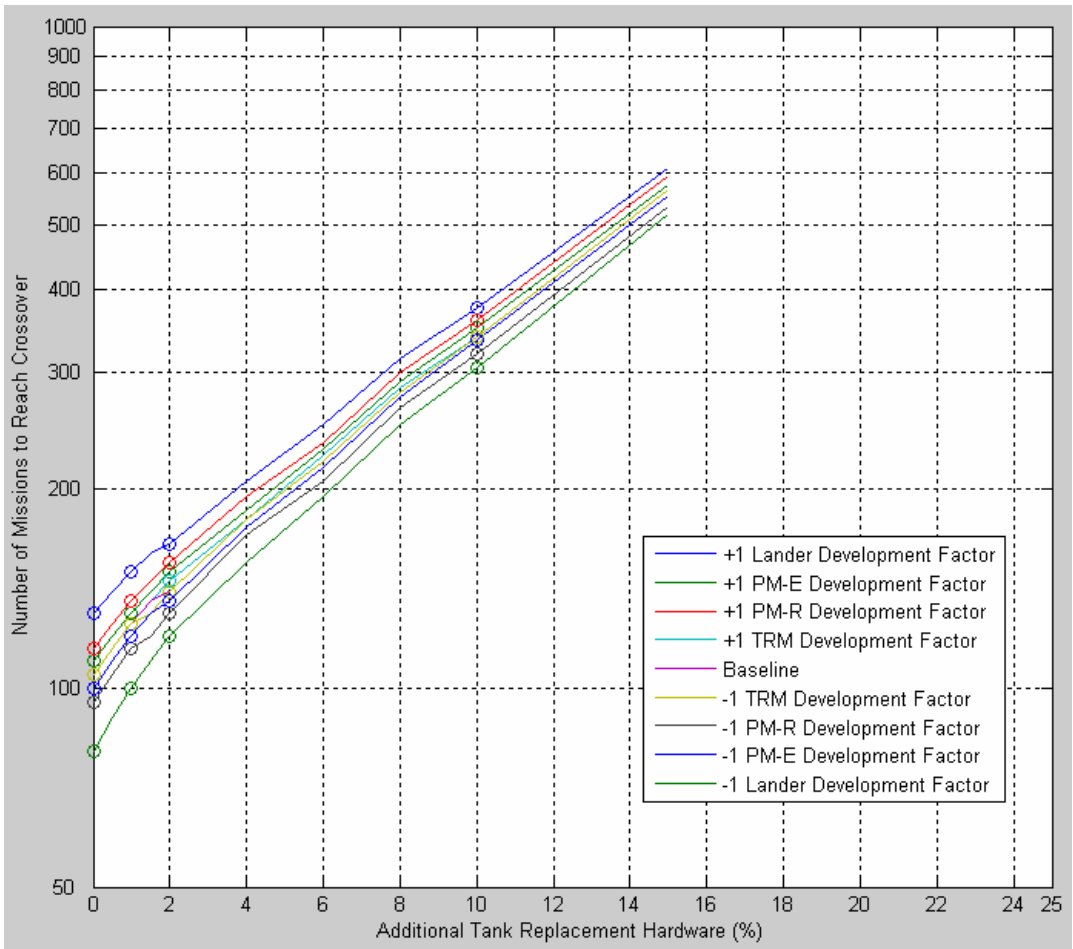

Figure 32: Tank replacement scenario sensitivity to development factor 


\section{Chapter 5: Conclusions}

\subsection{Significant Results}

The best choice of all of the architectures considered is NR-3; the no resupply lunar mission with a single expendable in-space propulsion module (PM-E), using direct return and reentry. This is a result of the number of expected missions to the moon to be limited to much less than 50 missions. More than likely, the number of missions will be less than 15 or 20 based on history and the potentially high cost of each mission. Also, the future goals of space travel are not entirely linked to crewed lunar missions but are focused on the moon as a near-term objective. This would drastically reduce the number of attempted missions to the moon, making re-supply less attractive for the moon. Section 5.2 discusses the advantages of pursuing resupply as a means for exploration of the moon and beyond.

However, other factors such as technology investment and future Martian insitu resource utilization (ISRU) would lead to a re-supply lunar architecture. Based on the analysis, the preferred choice for a re-supply architecture scenario is tank replacement. This is highly dependent on the intended lifetime of the lunar transportation system. If the number of missions to be attempted is similar to that of the Shuttle Transportation System (STS), then it is completely feasible to adopt some form of propellant re-supply as the means by which humans return to the moon. Propellant transfer is unlikely to be adopted as the preferred method because it requires about $40 \%$ more missions than tank replacement to be more cost effective than no re-supply. Although, propellant transfer does offer other advantages as far as operational and design simplicities. For example, all propellant transfer elements 
were the same size regardless of the transfer loss, which removes mass uncertainties from the architecture. Furthermore, the tank replacements method has a greater potential to reduce the lander gross mass. This is of particular importance since the lander is the single most expensive and sensitive element in all architectures. Based on reported propellant transfer losses, the additional hardware that must be added for the tank replacement option needs to be driven below $2 \%$ to stay more cost effective than propellant transfer. If the additional tank replacement hardware exceeds $2 \%$, then propellant transfer becomes more appealing.

The primary objective of the design of any lunar architecture needs to include accurate and reliable cost modeling. This is strongly evident in the substantial fluctuations in the results with minor alteration to the cost estimating parameters. In particular, the organizations managing and actually developing and producing the hardware need to be sufficiently advanced to maximize the amount of applicable experience for the necessary elements. Additionally, the production methods must be created in a way that ensures significant knowledge gained with the production of each new element; this is necessary to assure longer sustainability to reach the minimum number of missions. In particular the lander development and production must be strongly controlled. In strong contrast, the re-supply modules for the two methods can be allowed to grow more as needed because the crossover points were least sensitive to the propellant transfer module (PTM) and tank replacement module (TRM). 


\subsection{Additional Re-Supply Alternatives}

There are several mission alternatives to those proposed here. In particular, the desired area of exploration could be, and most likely will be, adjusted to other bodies of interest. New technologies could be introduced to phase in capability over several missions or to add safe abort and backup options.

The immediate focus of the lunar missions is to serve as a training ground and precursor for Martian exploration. For such missions, the $\Delta \mathrm{Vs}$ involved are much larger by 1.5 to 2 times. This large increase in $\Delta \mathrm{V}$ would cause the PMs to become exceedingly large, preventing any feasible mission scenario due to launch vehicle constraints. The use of propellant re-supply could be an important technology jump to facilitate the use of near-term launch and space vehicles. The PMs could grow by 2 or 3 times for a Mars-bound trajectory alone; the large vehicle increase is attributed to the larger $\Delta \mathrm{V}$ and additional safety protocols for the crew. Propellant re-supply would allow in-space propellant aggregation in LEO prior to the start of the mission to offset the large vehicle size. Re-supply would also facilitate the use of smaller vehicles for preparation of the return trajectory.

An important technology that is closely connected to the use of propellant resupply and Martian missions is ISRU. ISRU is useful because only the out-bound propellant would be necessary when leaving LEO; the in-bound propellant could be processed and delivered at the destination. However, one form of propellant resupply is necessary to refill the empty stages prior to Earth return. ISRU is a convenient technology that naturally suggests the development of re-supplying vehicles. 
The remaining location in which propellant re-supply is of particular interest is in the vicinity of Earth. Such technology advancements would encourage and support the use of reconnaissance satellites that must make regular orbital adjustments to view geographical areas of interest on the Earth. Since large orbit changes, such as plane changes and rendezvous, require large amounts of propellant, the current satellites conserve their propellant loading and are designed with large propellant storage tanks. Propellant re-supply could allow these satellites to be more liberal with the propellant usage and launch with smaller propellant loadings. Large space-based telescopes could gain from the propellant re-supply by being able to reposition themselves at will. This technology would also facilitate the development of ferry vehicles whose purpose is entirely based on taxiing satellites to and from high Earth orbit or geostationary orbits. This type of vehicle could substantially aid the capability of smaller, more affordable launch vehicles.

Propellant re-supply would also allow satellites to be built with more efficient propellant combinations. Currently, satellites must preserve their propellant by using storable propellants that do not boil in space. With the addition of re-supply, more efficient combinations using LOX could be utilized to enhance the satellite propulsion system. If propellant boils off, a re-supply module can top off the satellite tank. 


\section{$\underline{5.3 \text { Commercial Sector Alternatives }}$}

Currently, NASA and other government agencies maintain redundant individual capabilities, but such capabilities could be out-sourced to the private sector which continues to advance its means. Part of the newly developed expertise could be extended to support propellant aggregation and delivery to LEO or LLO. This would further reduce the burden of fulfilling propellant re-supply demands for lunar missions.

In the next several years, the number of companies that can deliver moderately sized payloads, on the order of 10 to 20 tons, to LEO will increase. This size payload would adequately deliver the PTM or TRM to re-supply any loitering assets in LEO. Combining the modest capability of smaller launch vehicles with the advanced propulsive vehicles owned by NASA and larger private companies would add the additional ability to re-supply the assets loitering in LLO. Even if the entire mission is directly controlled and monitored by NASA, small involvements of the private sector move to include and expand the private industry in the United States as well as potentially reducing the production and operational costs.

The other area of recent investment and boom for the private aerospace industry has been space tourism. Tourism and travel could be added as special capability and investment for the lunar missions. The ticket prices for lunar surface vacations or lunar orbital visits could be intertwined with science- and explorationbased missions to offset some of the infrastructure and operations costs. This would be comparable to the use of the Soyuz space vehicle and International Space Station by the Russian space agency to counteract reduced government spending in space. 
This is the semi-privatization of NASA much like that of the United States Postal Service: the government owns and operates the equipment, but the consumer must pay for the services.

Alternatively, the private industry would more likely invest in advanced technologies such as propellant transfer or tank replacements with the intention of completing the minimum number of missions necessary to gain a cost advantage. This is especially true of a space tourism company trying to corner the market against a tough competitor.

The final alternative is the use of launch vehicles that are developed and produced by private companies. This is in contrast to the SDLVs because the SDLVs would be developed by NASA and produced by private contractors through bids. Based on the current STS, the individual launch cost is minor compared to the nonrecurring costs of the system, as a result of several billion dollars being spent to maintain facilities and personnel regardless of the number of launches. Each launch throughout the year only adds a small incremental increase in costs to account for the actual hardware. The privately developed launch vehicles would be available for purchase based on a particular number of launches per year, i.e. NASA would contract to purchase 10 launch vehicles per year at a negotiated rate.

The added advantage of purchasing launch vehicles is the redundancy of other comparable launch vehicles. For example, if a launch failure occurs, even if the mission is completely lost, the next mission could be flown using a competing vehicle to avoid further delays while investigators determine the cause of the failure. Keeping with the NASA standard of protecting astronauts, the CEV and crew could 
still be launched on a government owned and operated vehicle; only allowing the cargo launches on the commercially acquired vehicle. 


\section{Appendices}

Listed below are all of the MATLAB scripts that were used.

Baseline.m

Calculates baseline crossover points for all architectures. Called by sensitivities.m.

Cell2Num.m

Converts cell-type arrays to a numeric array. Called in conjunction with ReadFromExcel.m

CompleteAnalysis.m

Mater driver to collect data from Microsoft Excel workbooks and perform development and production cost estimates for all architectures.

DevCostOutput.m

Exports all development cost data to Microsoft Excel workbooks using Write2Excel.m.

DevelopCosts.m

Function within Transcost.m to calculate the development costs of a vehicle, each stage, and engine.

FileOutput.m

Creates text version of development, production, and operations costs as a Transcost.m output option.

GenerateProductionNumbers.m

Calculates the total number of each type of element that needs to be produced for a given number of missions and architecture type.

Graphs_Cost.m

Generates all of the plots and figures for the baseline data and comparisons.

Graphs_Sens.m

Generates all of the plots and figures for the sensitivity study data.

NR_Devel_CostAnalysis.m

Function within CompleteAnaylsis.m to collect and format all no re-supply architecture data for use with Transcost.m and other functions. Data is collected from Microsoft Excel workbooks via ReadFromExcel.m. 
PrintOptions.m

Function within Transcost.m to manage user prompts.

ProductionCost.m

Function within Transcost.m to calculate the production costs of a vehicle, each stage, and engines.

PT Devel_CostAnalysis.m

Function within CompleteAnaylsis.m to collect and format all propellant transfer architecture data for use with Transcost.m and other functions. Data is collected from Microsoft Excel workbooks via ReadFromExcel.m.

ReadFromExcel.m

Written by Brett Shoelson, Ph.D. at the National Institute of Health. Collects data from specified Microsoft Excel workbooks, worksheets, and cells.

Save Sensitivities.m

Saves all sensitivity data to a Microsoft Excel workbook via Write2Excel.m

Sensitivities.m

Master driver to conduct sensitivity analyses and calculate crossover points for each.

Sensitivities_Development.m

Function within Sensitivities.m to calculate development and production data for each architecture with variations in development factor.

Sensitivities_Experience.m

Function within Sensitivities.m to calculate development and production data for each architecture with variations in the team experience factor.

Sensitivities InertMass.m

Function within Sensitivities.m to calculate development and production data for each architecture with variations in each vehicle inert mass.

Sensitivities_Learning.m

Function within Sensitivities.m to calculate development and production data for each architecture with variations in the learning curve factor.

Sensitivities_Reusability.m

Function within Sensitivities.m to calculate development and production data for each re-supply architecture with variations in the number of missions for which reusable elements can be used. 
Sensitivities_Reusability_Productions.m

Function within Sensitivities_Reusability.m to calculate production quantities for each element in each re-supply architecture.

TR_Devel_CostAnalysis.m

Function within CompleteAnaylsis.m to collect and format all tank replacement architecture data for use with Transcost.m and other functions. Data is collected from Microsoft Excel workbooks via ReadFromExcel.m.

\section{Transcost.m}

Master driver for Transcost cost estimating methodology.

Write2Excel.m

Written by Brett Shoelson, Ph.D. at the National Institute of Health. Sends data to specified Microsoft Excel workbooks, worksheets, and cells. 


\section{Glossary}

\begin{tabular}{|c|c|}
\hline CAD & Computer-Aided Design \\
\hline $\mathrm{CEV}$ & Crew Exploration Vehicle \\
\hline COTS & Commercial Off-The-Shelf \\
\hline EDL & Entry, Descent, and Landing \\
\hline EOC & Earth Orbit Capture \\
\hline EOR & Earth Orbit Rendezvous \\
\hline ESAS & Exploration Systems Architecture Study \\
\hline ISRU & In-Situ Resource Utilization \\
\hline LEO & Low Earth Orbit \\
\hline LLO & Low Lunar Orbit \\
\hline LOI & Lunar Orbit Insertion \\
\hline LOR & Lunar Orbit Rendezvous \\
\hline LOX & Liquid Oxygen \\
\hline MLI & Multi-Layer Insulation \\
\hline NR & No Re-supply \\
\hline OMS & Orbital Maneuvering System \\
\hline PM & In-Space Propulsion Module, Either Reusable or Expendable \\
\hline PM-E & Expendable In-Space Propulsion Module \\
\hline PM-R & Reusable In-Space Propulsion Module \\
\hline PT & Propellant Transfer \\
\hline PTM & Propellant Transfer Module \\
\hline
\end{tabular}




$\begin{array}{ll}\text { RCS } & \text { Reaction Control System } \\ \text { SDLV } & \text { Shuttle-Derived Launch Vehicle } \\ \text { S-IVb } & \text { Saturn V Upper Stage } \\ \text { SPSP } & \text { Space Propulsion Sizing Program } \\ \text { SPSP-L } & \text { Space Propulsion Sizing Program-Lander Version } \\ \text { SPSP-T } & \text { Space Propulsion Sizing Program-Tanker Version } \\ \text { STS } & \text { Shuttle Transportation System } \\ \text { TCM } & \text { Trajectory Correction Maneuver } \\ \text { TEI } & \text { Trans-Earth Injection } \\ \text { TLI } & \text { Trans-Lunar Injection } \\ \text { TR } & \text { Tank Replacement } \\ \text { TRM } & \text { Tank Replacement Module } \\ \Delta V & \text { Velocity Change Maneuver }\end{array}$




\section{References}

1. Smith, Richard K., "Seventy-Five Years of Inflight Refueling: Highlights, 1923-1998." Air Force History and Museums Program; 1998.

2. Griffin, J. W., "Background and Programmatic Approach for the Development of Orbital Fluid Resupply Tankers." AIAA Paper, AIAA 861601.

3. "Manned Lunar Landing Program Mode Comparison," NASA-TM-X-74929, July 30, 1962.

4. Boretz, J. E., “Orbital Refueling Techniques.” AIAA Paper, AIAA 69-564.

5. United States of America. National Aeronautics and Space Administration. "Exploration Systems Architecture Study: Final Report.” November 2005.

6. Scher, Michael D. and North, David, "The Space Propulsion Sizing Program." Space Systems Engineering Conference, Georgia Institute of Technology. GT.SSEC.F.6. November 2005.

7. United States of America. National Aeronautics and Space Administration. Johnson Space Center. "Design Mass Properties II: Mass Estimating and Forecasting for Aerospace Vehicles Based on Historical Data." JSC-26098, November 1994.

8. Koelle, Dietrich E., "Handbook of Cost Engineering for Space Transportation Systems with Transcost 7.1." TCS: TransCostSystems, Ottobrunn, Germany: 2003.

9. Klemetson, R. W., Garrison, P. W., and Hannum, N. P., “An Evaluation of Oxygen/Hydrogen Propulsion Systems for the Space Station.” AIAA Paper, AIAA-85-1156. 